TRANSACTIONS OF THE

AMERICAN MATHEMATICAL SOCIETY

Volume 356, Number 10, Pages 4085-4142

S 0002-9947(04)03467-1

Article electronically published on January 16, 2004

\title{
GREEN'S FUNCTIONS FOR ELLIPTIC AND PARABOLIC EQUATIONS WITH RANDOM COEFFICIENTS II
}

\author{
JOSEPH G. CONLON
}

\begin{abstract}
This paper is concerned with linear parabolic partial differential equations in divergence form and their discrete analogues. It is assumed that the coefficients of the equation are stationary random variables, random in both space and time. The Green's functions for the equations are then random variables. Regularity properties for expectation values of Green's functions are obtained. In particular, it is shown that the expectation value is a continuously differentiable function in the space variable whose derivatives are bounded by the corresponding derivatives of the Green's function for the heat equation. Similar results are obtained for the related finite difference equations. This paper generalises results of a previous paper which considered the case when the coefficients are constant in time but random in space.
\end{abstract}

\section{INTRODUCTION}

Let $(\Omega, \mathcal{F}, \mu)$ be a probability space and $\mathbf{a}: \Omega \rightarrow \mathbf{R}^{d(d+1) / 2}$ a bounded measurable function from $\Omega$ to the space of real symmetric $d \times d$ matrices. Let $I_{d}$ be the identity matrix in $d$ dimensions. We assume there are positive constants $\Lambda, \lambda$ such that

$$
\lambda I_{d} \leq \mathbf{a}(\omega) \leq \Lambda I_{d}, \quad \omega \in \Omega,
$$

in the sense of quadratic forms. Let $\mathbf{Z}$ denote the integers and $\mathbf{Z}^{d}$ the integer lattice in $d$ dimensions. We assume $\mathbf{Z}^{d} \times \mathbf{Z}$ acts on $\Omega$ by translation operators $\tau_{x, t}: \Omega \rightarrow \Omega, x \in \mathbf{Z}^{d}, t \in \mathbf{Z}$, which are measure preserving and satisfy the properties $\tau_{x, t} \tau_{y, s}=\tau_{x+y, t+s}, \tau_{0,0}=$ identity. For functions $g: \mathbf{Z}^{d} \times \mathbf{Z} \rightarrow \mathbf{R}$ we define the discrete derivative $\nabla_{i} g$ of $g$ in the ith direction, $1 \leq i \leq d$, to be

$$
\nabla_{i} g(x, t)=g\left(x+\mathbf{e}_{i}, t\right)-g(x, t), x \in \mathbf{Z}^{d}, t \in \mathbf{Z},
$$

where $\mathbf{e}_{i} \in \mathbf{Z}^{d}$ is the element with entry 1 in the ith position and 0 in the other positions. The gradient $\nabla g(x, t)$ of $g(x, t)$ is the column vector $\left(\nabla_{1} g(x, t), \ldots\right.$, $\left.\nabla_{d} g(x, t)\right)^{T}$. The formal adjoint of $\nabla_{i}$ is given by $\nabla_{i}^{*}$, where

$$
\nabla_{i}^{*} g(x, t)=g\left(x-\mathbf{e}_{i}, t\right)-g(x, t), x \in \mathbf{Z}^{d}, t \in \mathbf{Z} .
$$

The formal adjoint $\nabla^{*}$ of $\nabla$ then acts on vector fields $\mathbf{v}: \mathbf{Z}^{d} \times \mathbf{Z} \rightarrow \mathbf{R}^{d}$ by the formula

$$
\nabla^{*} \mathbf{v}(x, t)=\sum_{i=1}^{d} \nabla_{i}^{*} \mathbf{v}_{i}(x, t)
$$

Received by the editors July 23, 2002 and, in revised form, July 15, 2003.

2000 Mathematics Subject Classification. Primary 81T08, 82B20, 35R60, 60J75.

Key words and phrases. pde with random coefficients, homogenization. 
where

$$
\mathbf{v}(x, t)=\left(v_{1}(x, t), \ldots, v_{d}(x, t)\right)^{T} .
$$

We shall be interested in solutions to the initial value problem

$$
\begin{aligned}
& u(x, t+1, \omega)-u(x, t, \omega)=-\nabla^{*} \mathbf{a}\left(\tau_{x, t} \omega\right) \nabla u(x, t, \omega), \\
& x \in \mathbf{Z}^{d}, t \in \mathbf{Z}, t \geq 0, \omega \in \Omega, \\
& u(x, 0, \omega)=f(x, \omega), x \in \mathbf{Z}^{d}, \omega \in \Omega,
\end{aligned}
$$

where $f: \mathbf{Z}^{d} \times \Omega \rightarrow \mathbf{R}$ is a given measurable function. We may write the equation in (1.2) as

$$
u(x, t+1, \omega)=A_{t, \omega} u(x, t, \omega),
$$

where $A_{t, \omega}$ is a self-adjoint operator on $L^{2}\left(\mathbf{Z}^{d}\right)$. It is easy to see that if the constant $\Lambda$ of (1.1) satisfies $2 d \Lambda \leq 1$, then $A_{t, \omega}$ has norm $\left\|A_{t, \omega}\right\|$ with the property that $\left\|A_{t, \omega}\right\| \leq 1$. If $4 d \Lambda \leq 1$, then $A_{t, \omega}$ is also nonnegative definite. The solution to (1.2) can be written as

$$
u(x, t, \omega)=\sum_{y \in \mathbf{Z}^{d}} G_{\mathbf{a}}(x, y, t, \omega) f(y, \omega),
$$

where $G_{\mathbf{a}}(x, y, t, \omega)$ is the Green's function for (1.2). Denoting by $|\cdot|$ the Euclidean norm on $\mathbf{Z}^{d}$, it is clear that $G_{\mathbf{a}}(x, y, t, \omega)=0$ if $|x-y|>\sqrt{d} t$. It is easy to see also that

$$
\sum_{y \in \mathbf{Z}^{d}} G_{\mathbf{a}}(x, y, t, \omega)=1 .
$$

If $2 d \Lambda \leq 1$, then $\left\|A_{t, \omega}\right\| \leq 1$ implies

$$
\sum_{y \in \mathbf{Z}^{d}} G_{\mathbf{a}}(x, y, t, \omega)^{2} \leq 1 .
$$

Note that (1.4) implies (1.5) if the equation of (1.2) generates a Markov chain. Now let $G_{\mathbf{a}}(x, t), x \in \mathbf{Z}^{d}, t \in \mathbf{Z}, t>0$, be the expectation of the Green's function,

$$
\left\langle G_{\mathbf{a}}(x, y, t, \cdot)\right\rangle=G_{\mathbf{a}}(x-y, t) .
$$

From (1.4), (1.5) we have that if $2 d \Lambda \leq 1$, then

$$
\sum_{x \in \mathbf{Z}^{d}} G_{\mathbf{a}}(x, t)=1, \quad \sum_{x \in \mathbf{Z}^{d}} G_{\mathbf{a}}(x, t)^{2} \leq 1 .
$$

Let $|\cdot|_{\infty}$ be the norm on $\mathbf{Z}^{d}$ defined by $|x|_{\infty}=\sup \left\{\left|x_{1}\right|, \ldots,\left|x_{d}\right|\right\}$, for $x=\left(x_{1}, \ldots, x_{d}\right)$ $\in \mathbf{Z}^{d}$. We have then the following:

Theorem 1.1. Suppose $4 d \Lambda \leq 1$ and $x \in \mathbf{Z}^{d}, t \in \mathbf{Z}, t \geq 0$. Then there is $a$ universal constant $C>0$ and a constant $C_{d}$ depending only on $d$ such that:

(a) $\left|G_{\mathbf{a}}(x, t)\right| \leq \frac{C_{d}(\Lambda / \lambda)^{3 d+6}}{1+(\Lambda t)^{d / 2}} \exp \left[-\frac{\min \left\{|x|_{\infty},|x|_{\infty}^{2} /(1+\Lambda t)\right\}}{C}\right]$.

(b) $\left|\nabla G_{\mathbf{a}}(x, t)\right| \leq \frac{C_{d}(\Lambda / \lambda)^{3 d+6}}{1+(\Lambda t)^{d / 2}+1 / 2} \exp \left[-\frac{\min \left\{|x|_{\infty},|x|_{\infty}^{2} /(1+\Lambda t)\right\}}{C}\right]$.

Let $\delta$ satisfy $0 \leq \delta<1$. Then there is a constant $C_{d, \delta}$ depending only on $d$ and $\delta$ such that:

(c) $\left|G_{\mathbf{a}}(x, t+1)-G_{\mathbf{a}}(x, t)\right| \leq \frac{\Lambda^{\delta} C_{d, \delta}(\Lambda / \lambda)^{3 d+6}}{1+(\Lambda t)^{d / 2}+\delta} \exp \left[-\frac{\min \left\{|x|_{\infty},|x|_{\infty}^{2} /(1+\Lambda t)\right\}}{C}\right]$.

(d) $\left|\nabla \nabla G_{\mathbf{a}}(x, t)\right| \leq \frac{C_{d, \delta}(\Lambda / \lambda)^{3 d+6}}{1+(\Lambda t)^{d / 2}+\delta} \exp \left[-\frac{\min \left\{|x|_{\infty},|x|_{\infty}^{2} /(1+\Lambda t)\right\}}{C}\right]$. 
Remark 1. It is likely that the condition $4 d \Lambda \leq 1$ in Theorem 1.1 can be relaxed to $2 d \Lambda<1$.

Remark 2. The estimates in the proof of Theorem 1.1 (c) and (d) diverge as $\delta \rightarrow 1$.

Consider now the situation where there are translation operators on $\Omega$ which are continuous in time but discrete in space. Thus we assume there are translation operators $\tau_{x, t}: \Omega \rightarrow \Omega, x \in \mathbf{Z}^{d}, t \in \mathbf{R}$, which are measure preserving and satisfy the group properties $\tau_{x, t} \tau_{y, s}=\tau_{x+y, t+s}, \tau_{0,0}=$ identity. We also assume that the function from $\mathbf{R} \times \Omega$ to $\Omega$ defined by $(t, \omega) \rightarrow \tau_{0, t} \omega, t \in \mathbf{R}, \omega \in \Omega$, is measurable. Hence for every $x \in \mathbf{Z}^{d}$ the function $\mathbf{a}\left(\tau_{x, t} \omega\right)$ is a measurable function of $t \in \mathbf{R}$ with probability 1 in $\omega$. In analogy to (1.2) we can consider solutions to the initial value problem

$$
\begin{aligned}
\frac{\partial u}{\partial t}(x, t, \omega) & =-\nabla^{*} \mathbf{a}\left(\tau_{x, t} \omega\right) \nabla u(x, t, \omega), x \in \mathbf{Z}^{d}, t \in \mathbf{R}, t>0, \omega \in \Omega, \\
u(x, 0, \omega) & =f(x, \omega), x \in \mathbf{Z}^{d}, \omega \in \Omega,
\end{aligned}
$$

where $f: \mathbf{Z}^{d} \times \Omega \rightarrow \mathbf{R}$ is a given measurable function. As before, the solution may be written in the form (1.3) with the Green's function satisfying (1.4), (1.5). Defining now $G_{\mathbf{a}}(x, t), x \in \mathbf{Z}^{d}, t>0$, by (1.6), then it is easy to see that (1.7) holds. We have the following:

Theorem 1.2. Suppose $x \in \mathbf{Z}^{d}, t \in \mathbf{R}, t>0$. Then there is a universal constant $C>0$ and a constant $C_{d}$ depending only on $d$ such that:

(a) $\left|G_{\mathbf{a}}(x, t)\right| \leq \frac{C_{d}(\Lambda / \lambda)^{3 d+6}}{1+(\Lambda t)^{d / 2}} \exp \left[-\frac{\min \left\{|x|_{\infty},|x|_{\infty}^{2} /(1+\Lambda t)\right\}}{C}\right]$.

(b) $\left|\nabla G_{\mathbf{a}}(x, t)\right| \leq \frac{C_{d}(\Lambda / \lambda)^{3 d+6}}{1+(\Lambda t)^{d / 2+1 / 2}} \exp \left[-\frac{\min \left\{|x|_{\infty},|x|_{\infty}^{2} /(1+\Lambda t)\right\}}{C}\right]$.

Let $\delta$ satisfy $0 \leq \delta<1$. Then there is a constant $C_{d, \delta}$ depending only on $d$ and $\delta$ such that:

(c) $\left|G_{\mathbf{a}}(x, t)-G_{\mathbf{a}}\left(x, t^{\prime}\right)\right| \leq \frac{\left|t-t^{\prime}\right|^{\delta} \Lambda^{\delta} C_{d, \delta}(\Lambda / \lambda)^{3 d+6}}{1+(\Lambda t)^{d / 2+\delta}} \exp \left[-\frac{\min \left\{|x|_{\infty},|x|_{\infty}^{2} /(1+\Lambda t)\right\}}{c}\right]$, provided $\left|t-t^{\prime}\right|<t / 2$.

(d) $\left|\nabla \nabla G_{\mathbf{a}}(x, t)\right| \leq \frac{C_{d, \delta}(\Lambda / \lambda)^{3 d+6}}{1+(\Lambda t)^{d / 2}+\delta} \exp \left[-\frac{\min \left\{|x|_{\infty},|x|_{\infty}^{2} /(1+\Lambda t)\right\}}{C}\right]$.

Theorem 1.2 could be deduced directly from Theorem 1.1 if one could show that solutions of the discrete equation

$$
\begin{gathered}
\frac{u(x, t+\varepsilon, \omega)-u(x, t, \omega)}{\varepsilon}=-\nabla^{*} \mathbf{a}\left(\tau_{x, t} \omega\right) \nabla u(x, t, \omega), \\
x \in \mathbf{Z}^{d}, t \in \varepsilon \mathbf{Z}, t \geq 0, \omega \in \Omega,
\end{gathered}
$$

converge to solutions of the continuous equation (1.8) as $\varepsilon \rightarrow 0$. We prove the theorem in $\S 5$ by using the continuous version of certain formulas we have derived in the proof of Theorem 1.1 for discrete time. In this way we avoid the use of infinitesimal generators.

Finally, we consider the situation where there are translation operators on $\Omega$ which are continuous in both time and space. Thus we assume there are translation operators $\tau_{x, t}: \Omega \rightarrow \Omega, x \in \mathbf{R}^{d}, t \in \mathbf{R}$, which are measure preserving and satisfy the group properties $\tau_{x, t} \tau_{y, s}=\tau_{x+y, t+s}, \tau_{0,0}=$ identity. We also assume that the function from $\mathbf{R}^{d} \times \mathbf{R} \times \Omega$ to $\Omega$ defined by $(x, t, \omega) \rightarrow \tau_{x, t} \omega, x \in \mathbf{R}^{d}, t \in$ $\mathbf{R}, \omega \in \Omega$, is measurable. Hence the function $\mathbf{a}\left(\tau_{x, t} \omega\right)$ is a measurable function 
of $(x, t) \in \mathbf{R}^{d} \times \mathbf{R}$ with probability 1 in $\omega$. In analogy to (1.8) we can consider solutions to the initial value problem

$$
\begin{aligned}
\frac{\partial u}{\partial t}(x, t, \omega) & =-\nabla^{*} \mathbf{a}\left(\tau_{x, t} \omega\right) \nabla u(x, t, \omega), x \in \mathbf{R}^{d}, t \in \mathbf{R}, t>0, \omega \in \Omega, \\
u(x, 0, \omega) & =f(x, \omega), \quad x \in \mathbf{R}^{d}, \omega \in \Omega,
\end{aligned}
$$

where $f: \mathbf{R}^{d} \times \Omega \rightarrow \mathbf{R}$ is a given measurable function. The solution to (1.9) may be expressed as

$$
u(x, t, \omega)=\int_{\mathbf{R}^{d}} G_{\mathbf{a}}(x, y, t, \omega) f(y, \omega) d y,
$$

where the Green's function $G_{\mathbf{a}}(x, y, t, \omega)$ is a symmetric positive and positive definite kernel satisfying the identity

$$
\int_{\mathbf{R}^{d}} G_{\mathbf{a}}(x, y, t, \omega) d y=1 .
$$

We can now define the function $G_{\mathbf{a}}(x, t), x \in \mathbf{R}^{d}, t>0$, in terms of this Green's function by the equation (1.6). We have the following:

Theorem 1.3. Suppose $x \in \mathbf{R}^{d}, t \in \mathbf{R}, t>0$. Then there is a constant $C_{d}$ depending only on $d$ such that:

(a) $\left|G_{\mathbf{a}}(x, t)\right| \leq \frac{C_{d}(\Lambda / \lambda)^{3 d+6}}{(\Lambda t)^{d / 2}} \exp \left[-\frac{|x|^{2}}{8 \Lambda t}\right]$.

(b) $\left|\nabla G_{\mathbf{a}}(x, t)\right| \leq \frac{C_{d}(\Lambda / \lambda)^{3 d+6}}{(\Lambda t)^{d / 2}+1 / 2} \exp \left[-\frac{|x|^{2}}{8 \Lambda t}\right]$.

Let $\delta$ satisfy $0 \leq \delta<1$. Then there is a constant $C_{d, \delta}$ depending only on $d$ and $\delta$ such that:

(c) $\left|G_{\mathbf{a}}(x, t)-G_{\mathbf{a}}\left(x, t^{\prime}\right)\right| \leq \frac{\left|t-t^{\prime}\right|^{\delta} \Lambda^{\delta} C_{d, \delta}(\Lambda / \lambda)^{3 d+6}}{(\Lambda t)^{d / 2+\delta}} \exp \left[-\frac{|x|^{2}}{8 \Lambda t}\right]$, provided $\left|t-t^{\prime}\right|<t / 2, t^{\prime}<t$.

(d) $\left|\nabla G_{\mathbf{a}}(x, t)-\nabla G_{\mathbf{a}}\left(x^{\prime}, t\right)\right| \leq \frac{\left|x-x^{\prime}\right|^{2 \delta-1} C_{d, \delta}(\Lambda / \lambda)^{3 d+6}}{(\Lambda t)^{d / 2}+\delta} \exp \left[-\frac{|x|^{2}}{8 \Lambda t}\right]$, provided $\left|x-x^{\prime}\right|<|x| / 2,\left|x^{\prime}\right|>|x|, 1 / 2 \leq \delta<1$.

Theorem 1.3 could be deduced from Theorem 1.2 if one could show that solutions of discrete space lattice approximations to (1.9) converge to the solution of the pde (1.9) as the lattice spacing converges to zero. Theorem 1.3(a) is a consequence of the Aronson inequality [1] (see also [1], Appendix A). In [11] the factor $1 / 8$ also appears in the exponential bound. In [5], Chapter 3, an improvement of the factor $1 / 8$ to $1 / 4(1+\varepsilon), \varepsilon>0$, is obtained in the case when the coefficients in the diffusion equation are time independent. It seems likely that Theorem 1.1(a) and Theorem 1.2(a) should be a consequence of a discrete Aronson inequality. These discrete Aronson inequalities do not appear to be in the literature. See, however, [3 for discrete Aronson inequalities in the case of time independent coefficients, and [7] for an Aronson inequality in the case of time dependent coefficients.

The proofs of Theorems 1.1-1.3 do not require any ergodicity assumptions on the translation operators $\tau_{x, t},(x, t) \in \mathbf{R}^{d} \times \mathbf{R}$. In Lemma 2.5 we prove, under an ergodicity assumption, that a function related to the Fourier-Laplace transform of the expectation of the Green's function is continuous at zero energy and momentum. This fact is related to the existence of homogenization for equation (1.9). In [2] homogenization for a different equation was proven using the same strategy as in the proof of Lemma 2.5. A proof of homogenization for equation (1.9) was given in $[8]$. 
This paper is a sequel to 4. In that paper we proved inequalities for Green's functions in the case where the coefficients of the pde are time independent. Here we follow the same strategy as in [4], suitably modified to take into account the time dependence of the coefficients. We also keep more careful track of constants, whence we are able to show that the exponential decay rate of the expectation of the Green's function depends only on $\Lambda$.

Just recently it has been shown in some beautiful work of Delmotte and Deuschel [6] that one can take $\delta=1$ in parts (c) and (d) of Theorem 1.2 and Theorem 1.3. To prove this they work in configuration space instead of in Fourier space as is done in this paper and the previous one 4. They then use the Harnack inequality for parabolic equations, a deeper inequality than the interpolation theorems used here.

\section{FOURIER ANALYSIS}

We consider the function $G_{\mathbf{a}}(x, t), x \in \mathbf{Z}^{d}, t \in \mathbf{Z}, t \geq 0$, defined by (1.6). Our main goal here is to obtain a formula for the Fourier-Laplace transform $\hat{G}_{\mathbf{a}}(\xi, \eta)$ of $G_{\mathbf{a}}(x, t)$ and to prove some pointwise estimates for it. We define $\hat{G}_{\mathbf{a}}(\xi, \eta)$ for $\xi \in[-\pi, \pi]^{d}, \eta \in \mathbf{C}, \operatorname{Re}(\eta)>0$, by

$$
\hat{G}_{\mathbf{a}}(\xi, \eta)=\sum_{t=0}^{\infty} \sum_{x \in \mathbf{Z}^{d}} G_{\mathbf{a}}(x, t) \exp [i x \cdot \xi-\eta t] .
$$

Since $G_{\mathbf{a}}(x, t)=0$ if $|x|>\sqrt{2} t$ it follows from (1.7) that if $2 d \Lambda \leq 1$, then the RHS of (2.1) absolutely converges. It follows further that $\hat{G}_{\mathbf{a}}(\xi, \eta)$ is a $C^{\infty}$ function of $(\xi, \eta)$, analytic in $\eta$, and $G_{\mathbf{a}}(x, t)$ can be recovered from $\hat{G}_{\mathbf{a}}(\xi, \eta)$ by the inverse transform,

$$
G_{\mathbf{a}}(x, t)=\frac{1}{(2 \pi)^{d+1}} \int_{[-\pi, \pi]^{d}} d \xi e^{-i x \cdot \xi} \int_{-\pi}^{\pi} d[\operatorname{Im}(\eta)] e^{\eta t} \hat{G}_{\mathbf{a}}(\xi, \eta),
$$

where $\eta=\operatorname{Re}(\eta)+i \operatorname{Im}(\eta)$ and $\operatorname{Re}(\eta)>0$ is kept fixed in the integration.

To obtain a formula for $\hat{G}_{\mathbf{a}}(\xi, \eta)$ we proceed in a way similar to that developed in 4. We first rewrite the equation of (1.2) in terms of the function $v(x, t, \omega)$ defined by $v(x, t, \omega)=u\left(x, t, \tau_{-x,-t} \omega\right)$, where $u$ is the solution to the initial value problem (1.2). Hence $v(x, t, \omega)$ satisfies the equation

$$
\begin{aligned}
& \quad v\left(x, t+1, \tau_{0,1} \omega\right)-v(x, t, \omega) \\
& =-\sum_{i, j=1}^{d}\left\{a_{i, j}\left(\tau_{-\mathbf{e}_{j}, 0} \omega\right)\left[v\left(x+\mathbf{e}_{j}-\mathbf{e}_{i}, t, \tau_{\mathbf{e}_{j}-\mathbf{e}_{i}, 0} \omega\right)-v\left(x-\mathbf{e}_{i}, t, \tau_{-\mathbf{e}_{i}, 0} \omega\right)\right]\right. \\
& \left.-a_{i, j}(\omega)\left[v\left(x+\mathbf{e}_{j}, t, \tau_{\mathbf{e}_{j}, 0} \omega\right)-v(x, t, \omega)\right]\right\}=0, \\
& x \in \mathbf{Z}^{d}, t \in \mathbf{Z}, t \geq 0, \omega \in \Omega .
\end{aligned}
$$

From (1.2) the initial condition on $v(x, t, \omega)$ is $v(x, 0, \omega)=f\left(x, \tau_{-x, 0} \omega\right), x \in \mathbf{Z}^{d}$, $\omega \in \Omega$. Assume now that $f(x, \omega)=f(x)$ is independent of $\omega \in \Omega$ and has finite support in $\mathbf{Z}^{d}$. Then $v(x, t, \omega), x \in \mathbf{Z}^{d}$, also has finite support. Hence we may construct the Fourier transform $\hat{v}(\xi, t, \omega)$ of $v(x, t, \omega)$ by

$$
\hat{v}(\xi, t, \omega)=\sum_{x \in \mathbf{Z}^{d}} v(x, t, \omega) e^{i x \cdot \xi}, \xi \in[-\pi, \pi]^{d} .
$$


It is easy to see by Fourier transforming (2.3) that $\hat{v}(\xi, t, \omega)$ is the solution of an evolution equation.

We wish to write the equation for $\hat{v}(\xi, t, \omega)$ by using certain operators on functions $g: \Omega \rightarrow \mathbf{C}$ which are analogous to differential operators. For $1 \leq j \leq d$, $\xi \in[\pi, \pi]^{d}$ we define the $\xi$ derivative of $g$ in the $j$ th direction to be the function $\partial_{j, \xi} g$ given by

$$
\partial_{j, \xi} g(\omega)=e^{-i \mathbf{e}_{j} \cdot \xi} g\left(\tau_{\mathbf{e}_{j, 0}} \omega\right)-g(\omega), \omega \in \Omega .
$$

It is clear that $\partial_{j, \xi}$ is a bounded operator on $L^{2}(\Omega)$ with norm which does not exceed 2. The $\xi$ gradient $\partial_{\xi} g(\omega)$ of $g(\omega)$ is the column vector $\left(\partial_{1, \xi} g(\omega), \ldots, \partial_{d, \xi} g(\omega)\right)^{T}$. Let $\mathcal{H}(\Omega)$ be the Hilbert space of vector fields $\mathbf{v}: \Omega \rightarrow \mathbf{C}^{d}, \mathbf{v}(\omega)=\left(v_{1}(\omega), \ldots, v_{d}(\omega)\right)^{T}$ with the standard inner product. Then $\partial_{\xi}: L^{2}(\Omega) \rightarrow \mathcal{H}(\Omega)$ is a bounded operator. The adjoint $\partial_{\xi}^{*}: \mathcal{H}(\Omega) \rightarrow L^{2}(\Omega)$ of $\partial_{\xi}$ is given by

$$
\partial_{\xi}^{*} \mathbf{v}(\omega)=\sum_{j=1}^{d} \partial_{j, \xi}^{*} v_{j}(\omega),
$$

where $\partial_{j, \xi}^{*}$ is the adjoint of $\partial_{j, \xi}: L^{2}(\Omega) \rightarrow L^{2}(\Omega)$. From (2.4) we have that

$$
\partial_{j, \xi}^{*} g(\omega)=e^{i \mathbf{e}_{j} \cdot \xi} g\left(\tau_{-\mathbf{e}_{j}, 0} \omega\right)-g(\omega) .
$$

From (2.3) we see that $\hat{v}(\xi, t, \omega)$ satisfies the equation

$$
\begin{aligned}
\hat{v}\left(\xi, t+1, \tau_{0,1} \omega\right) & -\hat{v}(\xi, t, \omega) \\
& =-\partial_{\xi}^{*} \mathbf{a}(\omega) \partial_{\xi} \hat{v}(\xi, t, \omega), \xi \in[-\pi, \pi]^{d}, \quad t \in \mathbf{Z}, t \geq 0, \omega \in \Omega .
\end{aligned}
$$

The initial condition is given by $\hat{v}(\xi, 0, \omega)=\hat{f}(\xi), \xi \in[-\pi, \pi]^{d}, t \geq 0, \omega \in \Omega$.

Since $f$ has finite support we may also take the Laplace transform of $\hat{v}(\xi, t, \omega)$. Thus for $\eta$ satisfying $\operatorname{Re}(\eta)>0$, we put

$$
\hat{v}(\xi, \eta, \omega)=\sum_{t=0}^{\infty} \hat{v}(\xi, t, \omega) e^{-\eta t} .
$$

From (2.5) and the fact that $\hat{v}(\xi, 0, \omega)=\hat{f}(\xi)$, we see that $\hat{v}(\xi, \eta, \omega)$ satisfies the equation

$$
\begin{aligned}
& e^{\eta} \hat{v}\left(\xi, \eta, \tau_{0,1} \omega\right)-\hat{v}(\xi, \eta, \omega)+\partial_{\xi}^{*} \mathbf{a}(\omega) \partial_{\xi} \hat{v}(\xi, \eta, \omega) \\
& =e^{\eta} \hat{f}(\xi), \quad \xi \in[-\pi, \pi]^{d}, \quad \operatorname{Re}(\eta)>0, \omega \in \Omega .
\end{aligned}
$$

It is easy to see that $\hat{v}(\xi, \eta, \omega)$ is related to $\hat{G}_{\mathbf{a}}(\xi, \eta)$ by the identity

$$
\langle\hat{v}(\xi, \eta, \cdot)\rangle=\hat{G}_{\mathbf{a}}(\xi, \eta) \hat{f}(\xi), \xi \in[-\pi, \pi]^{d}, \operatorname{Re}(\eta)>0 .
$$

We can use (2.6), (2.7) to obtain an estimate on $\hat{G}_{\mathbf{a}}(\xi, \eta)$ provided $2 d \Lambda \leq 1$.

Lemma 2.1. Suppose $2 d \Lambda \leq 1$. Then $\hat{G}_{\mathbf{a}}(\xi, \eta)$ satisfies the inequality

$$
\left|\hat{G}_{\mathbf{a}}(\xi, \eta)\right| \leq e^{\operatorname{Re}(\eta)} /\left[e^{\operatorname{Re}(\eta)}-1\right], \quad \xi \in[-\pi, \pi]^{d}, \quad \operatorname{Re}(\eta)>0 .
$$


Proof. We multiply (2.6) by $\exp [-i \operatorname{Im}(\eta)] \overline{\hat{v}\left(\xi, \eta, \tau_{0,1} \omega\right)}$ and take the expectation value. Thus we obtain the equation

$$
\begin{aligned}
e^{\operatorname{Re}(\eta)}\left\langle|\hat{v}(\xi, \eta, \cdot)|^{2}\right\rangle & =e^{\operatorname{Re}(\eta)} \overline{\hat{G}_{\mathbf{a}}(\xi, \eta)}|\hat{f}(\xi)|^{2} \\
& +\exp [-i \operatorname{Im}(\eta)]\left\langle\overline{\hat{v}\left(\xi, \eta, \tau_{0,1} \cdot\right)}\left[I-\partial_{\xi}^{*} \mathbf{a}(\cdot) \partial_{\xi}\right] \hat{v}(\xi, \eta, \cdot)\right\rangle .
\end{aligned}
$$

Observe now that for any function $g \in L^{2}(\Omega)$ there is the inequality

$$
0 \leq\left\langle\overline{\partial_{\xi} g(\cdot)} \mathbf{a}(\cdot) \partial_{\xi} g(\cdot)\right\rangle=\left\langle\overline{g(\cdot)} \partial_{\xi}^{*} \mathbf{a}(\cdot) \partial_{\xi} g(\cdot)\right\rangle \leq 4 d \Lambda\left\langle|g(\cdot)|^{2}\right\rangle \leq 2\left\langle|g(\cdot)|^{2}\right\rangle .
$$

It follows that $I-\partial_{\xi}^{*} \mathbf{a}(\cdot) \partial_{\xi}$ is a self-adjoint operator on $L^{2}(\Omega)$ with spectrum in the interval $[-1,1]$. Hence the Schwarz inequality applied to the RHS of (2.8) yields the inequality

$$
\left[e^{\operatorname{Re}(\eta)}-1\right]\left\langle|\hat{v}(\xi, \eta, \cdot)|^{2}\right\rangle \leq e^{\operatorname{Re}(\eta)}\left|\hat{G}_{\mathbf{a}}(\xi, \eta)\right||\hat{f}(\xi)|^{2} .
$$

If we combine the previous inequality with Jensen's inequality and (2.7), we obtain

$$
\left[e^{R e(\eta)}-1\right]\left|\hat{G}_{\mathbf{a}}(\xi, \eta)\right|^{2}|\hat{f}(\xi)|^{2} \leq e^{R e(\eta)}\left|\hat{G}_{\mathbf{a}}(\xi, \eta)\right||\hat{f}(\xi)|^{2} .
$$

Our only requirement on $f$ is that it has finite support. Since functions of finite support are dense in $L^{2}\left(\mathbf{Z}^{d}\right)$ the result follows.

We can obtain sharper estimates on $\hat{G}_{\mathbf{a}}(\xi, \eta)$ than that given in Lemma 2.1. To do this we write

$$
\hat{v}(\xi, \eta \omega):=\hat{u}(\xi, \eta)+\hat{\psi}(\xi, \eta, \omega),
$$

where $\langle\hat{\psi}(\xi, \eta, \cdot)\rangle=0$. If we subtract the expectation value of (2.6) from (2.6) we obtain the equation

$(2.10) e^{\eta} \hat{\psi}\left(\xi, \eta, \tau_{0,1} \omega\right)-\hat{\psi}(\xi, \eta, \omega)+P \partial_{\xi}^{*} \mathbf{a}(\omega) \partial_{\xi} \hat{\psi}(\xi, \eta, \omega)=\hat{u}(\xi, \eta) P \partial_{\xi}^{*} \mathbf{a}(\omega) e(-\xi)$,

where $P: L^{2}(\Omega) \rightarrow L^{2}(\Omega)$ is the projection orthogonal to the constant function. The column vector $e(\xi)=\left(e_{1}(\xi), \ldots, e_{d}(\xi)\right)^{T}, \xi \in[-\pi, \pi]^{d}$, has entries $e_{k}(\xi)$ given by

$$
e_{k}(\xi)=1-\exp \left[i \mathbf{e}_{k} \cdot \xi\right], \quad 1 \leq k \leq d .
$$

Suppose now $\Phi(\xi, \eta, \omega)=\left(\Phi_{1}(\xi, \eta, \omega), \ldots, \Phi_{d}(\xi, \eta, \omega)\right)$ is the row vector which satisfies the equation

$$
e^{\eta} \Phi\left(\xi, \eta, \tau_{0,1} \omega\right)-\Phi(\xi, \eta, \omega)+P \partial_{\xi}^{*} \mathbf{a}(\omega) \partial_{\xi} \Phi(\xi, \eta, \omega)+P \partial_{\xi}^{*} \mathbf{a}(\omega)=0 .
$$

If the solutions of (2.10), (2.11) are unique, then we must have

$$
\hat{\psi}(\xi, \eta, \omega)=-\hat{u}(\xi, \eta) \Phi(\xi, \eta, \omega) e(-\xi) .
$$

If we substitute this last formula together with (2.9) into (2.6) and take the expectation value, we obtain the identity

$$
\begin{gathered}
\hat{u}(\xi, \eta)\left[e^{\eta}-1+e(\xi)\langle\mathbf{a}(\cdot)\rangle e(-\xi)+e(\xi)\left\langle\mathbf{a}(\cdot) \partial_{\xi} \Phi(\xi, \eta, \cdot)\right\rangle e(-\xi)\right] \\
=e^{\eta} \hat{f}(\xi) .
\end{gathered}
$$

Comparing this last expression with (2.7), we conclude that $\hat{G}_{\mathbf{a}}(\xi, \eta)$ is given by the formula

$$
\hat{G}_{\mathbf{a}}(\xi, \eta)=e^{\eta} /\left[e^{\eta}-1+e(\xi) q(\xi, \eta) e(-\xi)\right],
$$


where $q(\xi, \eta)$ is the $d \times d$ matrix

$$
q(\xi, \eta)=\langle\mathbf{a}(\cdot)\rangle+\left\langle\mathbf{a}(\cdot) \partial_{\xi} \Phi(\xi, \eta, \cdot)\right\rangle .
$$

The formulas (2.12), (2.13) for the expectation of the Fourier-Laplace transform of the Green's function hold provided we can prove existence and uniqueness of solutions to (2.10), (2.11). We establish this next.

Lemma 2.2. Suppose $2 d \Lambda \leq 1, \operatorname{Re}(\eta)>0, \xi \in[-\pi, \pi]^{d}$ and $\varphi \in L^{2}(\Omega)$. Then there is a unique solution $\psi \in L^{2}(\Omega)$ to the equation

$$
e^{\eta} \psi\left(\tau_{0,1} \omega\right)-\psi(\omega)+P \partial_{\xi}^{*} \mathbf{a}(\omega) \partial_{\xi} \psi(\omega)=\varphi(\omega), \quad \omega \in \Omega .
$$

Further, if $\langle\varphi\rangle=0$, then $\langle\psi\rangle=0$.

Proof. Observe that if $\psi$ satisfies (2.14), then

$$
\left(e^{\eta}-1\right)\langle\psi\rangle=\langle\varphi\rangle .
$$

Since $\operatorname{Re}(\eta)>0$, it follows that $\langle\varphi\rangle=0$ implies $\langle\psi\rangle=0$. We also can see that if $\psi$ satisfies (2.14), then $\psi$ also satisfies the equation

$$
e^{\eta} \psi\left(\tau_{0,1} \omega\right)=P\left[I-\partial_{\xi}^{*} \mathbf{a}(\omega) \partial_{\xi}\right] \psi(\omega)+\frac{e^{\eta}}{e^{\eta}-1}\langle\varphi\rangle+P \varphi(\omega) .
$$

Conversely, if $\psi$ satisfies (2.15), then it satisfies (2.14) as well. We already observed in Lemma 2.1 that the operator $I-\partial_{\xi}^{*} \mathbf{a}(\omega) \partial_{\xi}$ on $L^{2}(\Omega)$ has norm which does not exceed 1. Hence the Lax-Milgram theorem implies that (2.15) has a unique solution $\psi \in L^{2}(\Omega)$.

In Lemma 2.2 the estimate on the norm of the solution $\psi$ to (2.14) diverges as $\operatorname{Re}(\eta) \rightarrow 0$. In the following lemma we show that the norm of the $\xi$ gradient of $\psi$ can remain finite as $\operatorname{Re}(\eta) \rightarrow 0$.

Lemma 2.3. Suppose $4 d \Lambda \leq 1, \operatorname{Re}(\eta)>0, \xi \in[-\pi, \pi]^{d}$ and $\varphi \in \mathcal{H}(\Omega)$. Let $\psi \in L^{2}(\Omega)$ be the solution to the equation

$$
e^{\eta} \psi\left(\tau_{0,1} \omega\right)-\psi(\omega)+P \partial_{\xi}^{*} \mathbf{a}(\omega) \partial_{\xi} \psi(\omega)=P \partial_{\xi}^{*} \varphi(\omega), \quad \omega \in \Omega .
$$

Then there is the inequality

$$
\left[e^{R e(\eta)}-1\right]\left\langle|\psi|^{2}\right\rangle+\frac{1}{2}\left\langle\overline{\partial_{\xi} \psi(\cdot)} \mathbf{a}(\cdot) \partial_{\xi} \psi(\cdot)\right\rangle \leq \frac{1}{2}\left\langle\overline{\varphi(\cdot)} \mathbf{a}(\cdot)^{-1} \varphi(\cdot)\right\rangle .
$$

Proof. We multiply $(2.16)$ by $\exp [-i \operatorname{Im}(\eta)] \overline{\psi\left(\tau_{0,1} \omega\right)}$ and take the expectation value. Since $\langle\psi\rangle=0$ we have

$$
\begin{aligned}
e^{\operatorname{Re}(\eta)}\left\langle|\psi|^{2}\right\rangle & =e^{-i \operatorname{Im}(\eta)}\left\langle\overline{\psi\left(\tau_{0,1} \cdot\right)}\left[I-\partial_{\xi}^{*} \mathbf{a}(\cdot) \partial_{\xi}\right] \psi(\cdot)\right\rangle \\
& +e^{-i \operatorname{Im}(\eta)}\left\langle\overline{\partial_{\xi} \psi\left(\tau_{0,1} \cdot\right)} \varphi(\cdot)\right\rangle .
\end{aligned}
$$

Since $4 d \Lambda \leq 1$ the self-adjoint operator $I-\partial_{\xi}^{*} \mathbf{a}(\cdot) \partial_{\xi}$ on $L^{2}(\Omega)$ is nonnegative definite. Hence, applying the Schwarz inequality to the RHS of (2.18) we have

$$
\begin{aligned}
e^{\operatorname{Re}(\eta)}\left\langle|\psi|^{2}\right\rangle & \leq \frac{1}{2}\left\langle\overline{\psi\left(\tau_{0,1} \cdot\right)}\left[I-\partial_{\xi}^{*} \mathbf{a}(\cdot) \partial_{\xi}\right] \psi\left(\tau_{0,1} \cdot\right)\right\rangle+\frac{1}{2}\left\langle\overline{\psi(\cdot)}\left[I-\partial_{\xi}^{*} \mathbf{a}(\cdot) \partial_{\xi}\right] \psi(\cdot)\right\rangle \\
& +\frac{1}{2}\left\langle\overline{\psi\left(\tau_{0,1} \cdot\right)} \partial_{\xi}^{*} \mathbf{a}(\cdot) \partial_{\xi} \psi\left(\tau_{0,1} \cdot\right)\right\rangle+\frac{1}{2}\left\langle\overline{\varphi(\cdot)} \mathbf{a}(\cdot)^{-1} \varphi(\cdot)\right\rangle .
\end{aligned}
$$

The inequality (2.17) follows from this last inequality. 
We can use Lemma 2.3 to establish regularity of the matrix $q(\xi, \eta)$ of (2.13).

Lemma 2.4. Suppose $4 d \Lambda \leq 1, \operatorname{Re}(\eta)>0, \xi \in[-\pi, \pi]^{d}$. Then $q(\xi, \eta)$ is a $C^{\infty}$ function of $(\xi, \eta)$, periodic in $\xi$, and analytic in $\eta$.

Proof. For $\alpha$ satisfying $0<4 d \alpha \leq 1, \operatorname{Re}(\eta)>0, \xi \in[-\pi, \pi]^{d}$ we define an operator $T_{\alpha, \eta, \xi}$ on $\mathcal{H}(\Omega)$ by

$$
T_{\alpha, \eta, \xi} \varphi=\alpha \partial_{\xi} \psi, \quad \varphi \in \mathcal{H}(\Omega),
$$

where $\psi$ is the solution to the equation

$$
e^{\eta} \psi\left(\tau_{0,1} \omega\right)-\psi(\omega)+\alpha \partial_{\xi}^{*} \partial_{\xi} \psi(\omega)=\partial_{\xi}^{*} \varphi(\omega), \quad \omega \in \Omega .
$$

By the argument of Lemma 2.2 we see that $\psi \in L^{2}(\Omega)$ is unique. By the argument of Lemma 2.3 we see that $T_{\alpha, \eta, \xi}$ is a bounded operator on $\mathcal{H}(\Omega)$ with norm satisfying $\left\|T_{\alpha, \eta, \xi}\right\| \leq 1$. We put $\mathbf{b}(\omega)=\left[\Lambda I_{d}-\mathbf{a}(\omega)\right] / \Lambda, \omega \in \Omega$. From (1.1) $\mathbf{b}(\omega)$ is a real symmetric matrix satisfying the inequality

$$
0 \leq \mathbf{b}(\omega) \leq\left[1-\frac{\lambda}{\Lambda}\right] I_{d}, \quad \omega \in \Omega
$$

in the sense of quadratic forms. Note now that the solution $\psi$ of (2.16) also satisfies the equation

$$
\begin{aligned}
\partial_{\xi} \psi(\omega) & -T_{\Lambda, \eta, \xi} P \mathbf{b}(\omega) \partial_{\xi} \psi(\omega) \\
& =\frac{1}{\Lambda} T_{\Lambda, \eta, \xi} P \varphi(\omega), \quad \omega \in \Omega .
\end{aligned}
$$

In view of (2.21) and the fact that $\left\|T_{\Lambda, \eta, \xi}\right\| \leq 1$ equation (2.22) is uniquely solvable for $\partial_{\xi} \psi \in \mathcal{H}(\Omega)$.

Let $\mathcal{M}$ be the space of complex-valued $d \times d$ matrices $A$ with adjoint $A^{*}$. We define the space $L^{2}(\Omega, \mathcal{M})$ to be the space of measurable functions $A: \Omega \rightarrow \mathcal{M}$ with norm

$$
\|A\|^{2}=\left\langle\operatorname{Tr} A^{*}(\cdot) A(\cdot)\right\rangle
$$

Now the operator $T_{\Lambda, \eta, \xi}$ on $\mathcal{H}(\Omega)$ can be extended to an operator on $L^{2}(\Omega, \mathcal{M})$ by simply allowing $T_{\Lambda, \eta, \xi}$ to act on each column vector of $A(\omega)$. It is easy to see then that $T_{\Lambda, \eta, \xi}$ is a bounded operator on $L^{2}(\Omega, \mathcal{M})$ and $\left\|T_{\Lambda, \eta, \xi}\right\| \leq 1$. Let $\Psi(\xi, \eta, \omega)=\partial_{\xi} \Phi(\xi, \eta, \omega) \in \mathcal{M}$, where $\Phi$ is the solution of (2.11). Comparing (2.11), (2.16), (2.22) we see that $\Psi(\xi, \eta, \omega)$ satisfies the equation

$$
\begin{aligned}
\Psi(\xi, \eta, \omega) & -T_{\Lambda, \eta, \xi} P \mathbf{b}(\omega) \Psi(\xi, \eta, \omega) \\
& +\frac{1}{\Lambda} T_{\Lambda, \eta, \xi} P \mathbf{a}(\omega)=0, \quad \omega \in \Omega .
\end{aligned}
$$

From (2.13) we see that $q(\xi, \eta)$ is given by the formula

$$
q(\xi, \eta)=\langle\mathbf{a}(\cdot)\rangle+\langle\mathbf{a}(\cdot) \Psi(\xi, \eta, \cdot)\rangle .
$$

In view of (2.21) it follows that $\Psi(\xi, \eta, \cdot) \in L^{2}(\Omega, \mathcal{M})$, whence (2.24) implies $q(\xi, \eta)$ is a finite matrix. 
In order to prove regularity of $q(\xi, \eta)$ we obtain a representation for the operator $T_{\alpha, \eta, \xi}$ defined by (2.19), (2.20). To do this we let $G_{\alpha}(x, t)$ be the Green's function for (1.2) in the case $\mathbf{a}(\omega)=\alpha I_{d}$. Thus $G_{\alpha}(x, t)$ satisfies the initial value problem

$$
\begin{array}{r}
G_{\alpha}(x, t+1)-G_{\alpha}(x, t)+\alpha \nabla^{*} \nabla G_{\alpha}(x, t)=0, x \in \mathbf{Z}^{d}, t \in \mathbf{Z}, t \geq 0, \\
G_{\alpha}(x, 0)=\delta(x), \quad x \in \mathbf{Z}^{d},
\end{array}
$$

where $\delta(x)$ is the Kronecker $\delta$ function, $\delta(0)=1, \delta(x)=0, x \neq 0$. The Fourier transform $\hat{G}_{\alpha}(\xi, t)$ of $G_{\alpha}(x, t)$ is evidently given by

$$
\hat{G}_{\alpha}(\xi, t)=\left[1-\alpha|e(\xi)|^{2}\right]^{t}, \xi \in[-\pi, \pi]^{d}, t \in \mathbf{Z}, t \geq 0 .
$$

The Fourier-Laplace transform $\hat{G}_{\alpha}(\xi, \eta)$ of $G_{\alpha}(x, t)$ is given by

$$
\hat{G}_{\alpha}(\xi, \eta)=e^{\eta} /\left[e^{\eta}-1+\alpha|e(\xi)|^{2}\right], \xi \in[-\pi, \pi]^{d}, \operatorname{Re}(\eta)>0 .
$$

The function $G_{\alpha}(x, t)$ satisfies an inequality

$$
0 \leq G_{\alpha}(x, t) \leq \frac{C_{d}}{1+(\alpha t)^{d / 2}} \exp \left[-\frac{\min \left\{|x|,|x|^{2} /(1+\alpha t)\right\}}{C_{d}}\right],
$$

where $C_{d}>0$ is a constant depending only on the dimension $d$. Observe now that by the argument of Lemma 2.2 the equation

$$
e^{\eta} \psi\left(\tau_{0,1} \omega\right)-\psi(\omega)+\alpha \partial_{\xi}^{*} \partial_{\xi} \psi(\omega)=\varphi(\omega), \quad \omega \in \Omega,
$$

has a unique solution $\psi \in L^{2}(\Omega)$ provided $\varphi \in L^{2}(\Omega), \operatorname{Re}(\eta)>0, \xi \in[-\pi, \pi]^{d}, 2 d \alpha$ $\leq 1$. The formulas (2.26), (2.27) also hold for the same range of values $\eta, \xi, \alpha$. Consider now the function $\psi(\omega)$ given by

$$
\psi(\omega)=\sum_{t=0}^{\infty} e^{-\eta(t+1)} \sum_{x \in Z^{d}} G_{\alpha}(x, t) e^{-i x \cdot \xi} \varphi\left(\tau_{x,-t-1} \omega\right), \quad \omega \in \Omega .
$$

It is clear that if $\varphi \in L^{2}(\Omega), \operatorname{Re}(\eta)>0, \xi \in[-\pi, \pi]^{d}, 2 d \alpha \leq 1$, then $\psi \in L^{2}(\Omega)$ and $\psi$ is a solution to (2.28). It follows from (2.19), (2.20), (2.28), (2.29) that the operator $T_{\alpha, \eta, \xi}$ is given by the formula

$$
T_{\alpha, \eta, \xi} \varphi(\omega)=\alpha \sum_{t=0}^{\infty} e^{-\eta(t+1)} \sum_{x \in Z^{d}} \nabla \nabla^{*} G_{\alpha}(x, t) e^{-i x \cdot \xi} \varphi\left(\tau_{x,-t-1} \omega\right), \quad \omega \in \Omega
$$

where $\nabla \nabla^{*} G_{\alpha}(x, t)$ denotes the $d \times d$ matrix with entry in the $i$ th row and $j$ th column given by $\nabla_{j} \nabla_{i}^{*} G_{\alpha}(x, t)$.

We shall conclude the regularity of $q(\xi, \eta)$ from the regularity of $\Psi(\xi, \eta, \cdot)$ regarded as a mapping from $[-\pi, \pi]^{d} \times\{\operatorname{Re}(\eta)>0\}$ to $L^{2}(\Omega, \mathcal{M})$. From (2.23) $\Psi(\xi, \eta, \cdot)$ can be written as a Neumann series,

$$
\Psi(\xi, \eta, \cdot)=\sum_{n=1}^{\infty}\left[T_{\Lambda, \eta, \xi} P \mathbf{b}(\cdot)\right]^{n} .
$$


From (2.30) we may define the derivative operator of $T_{\alpha, \eta, \xi}$ with respect to $\eta$ by

$$
\begin{aligned}
& \frac{\partial}{\partial \eta} T_{\alpha, \eta, \xi} \varphi(\omega)=-\alpha \sum_{t=0}^{\infty}(t+1) e^{-\eta(t+1)} \\
& \quad \sum_{x \in Z^{d}} \nabla \nabla^{*} G_{\alpha}(x, t) e^{-i x \cdot \xi} \varphi\left(\tau_{x,-t-1} \omega\right), \quad \omega \in \Omega .
\end{aligned}
$$

It follows from (2.27) that $\partial / \partial \eta T_{\alpha, \eta, \xi}$ is a bounded operator on $\mathcal{H}(\Omega)$ and $L^{2}(\Omega, \mathcal{M})$. From (2.31), 2.32) we see that $\Psi(\xi, \eta, \cdot)$ is differentiable with respect to $\eta$ and its derivative is given by the formula

$$
\begin{aligned}
& \frac{\partial \Psi}{\partial \eta}(\xi, \eta, \cdot)=\sum_{n=0}^{\infty} \sum_{m=0}^{n}\left[T_{\Lambda, \eta, \xi} P \mathbf{b}(\cdot)\right]^{m} \\
& \cdot\left[\frac{\partial}{\partial \eta} T_{\Lambda, \eta, \xi} P \mathbf{b}(\cdot)\right]\left[T_{\Lambda, \eta, \xi} P \mathbf{b}(\cdot)\right]^{n-m}
\end{aligned}
$$

We can see similarly that $\Psi(\xi, \eta, \cdot)$ is $C^{\infty}$ in $(\xi, \eta)$ and analytic in $\eta$.

Lemma 2.5. Suppose $4 d \Lambda \leq 1, \operatorname{Re}(\eta)>0, \xi \in[-\pi, \pi]^{d}$. Then the limit $q(\xi, 0)$ $=\lim _{\eta \rightarrow 0} q(\xi, \eta)$ exists for all $\xi \in[-\pi, \pi]^{d}$. If any of the translation operators $\tau_{\mathbf{e}_{i}, 0}, 1 \leq i \leq d$, or $\tau_{0,1}$ are ergodic on $\Omega$, then $q(\xi, 0)$ is continuous at $\xi=0$.

Proof. We show that the solution $\Psi(\xi, \eta, \cdot)$ of $[2.23)$ converges for fixed $\xi \in[-\pi, \pi]^{d}$ to a function $\Psi(\xi, 0, \cdot) \in L^{2}(\Omega, \mathcal{M})$ as $\eta \rightarrow 0$. To see this we use the representation (2.31). We shall define an operator $T_{\alpha, 0, \xi}$ on $L^{2}(\Omega, \mathcal{M})$ provided $0<4 d \alpha \leq 1$. Then $\Psi(\xi, 0, \cdot)$ is given by the series (2.31) with $\eta=0$.

To prove the existence of the operator $T_{\alpha, 0, \xi}$ we argue as in Lemma 3.1 of [4]. In view of (2.19), (2.20) we define

$$
T_{\alpha, 0, \xi} \varphi=0, \quad \varphi \in \mathcal{H}(\Omega), \quad \partial_{\xi}^{*} \varphi=0 .
$$

From (2.30) we define

$$
\begin{aligned}
& T_{\alpha, \eta, \xi} \varphi(\omega)=\alpha \sum_{t=0}^{\infty} \sum_{x \in \mathbf{Z}^{d}}\left(\nabla^{*} \nabla\right) \nabla^{*} G_{\alpha}(x, t) e^{-i x \cdot \xi} \psi\left(\tau_{x,-t-1} \omega\right), \\
& \omega \in \Omega, \varphi=\partial_{\xi} \psi, \quad \psi \in L^{2}(\Omega) .
\end{aligned}
$$

In view of the inequality

$$
\left|\left(\nabla^{*} \nabla\right) \nabla^{*} G_{\alpha}(x, t)\right| \leq \frac{C_{d}}{1+(\alpha t)^{(d+3) / 2}} \exp \left[-\frac{\min \left\{|x|,|x|^{2} /(1+\alpha t)\right\}}{C_{d}}\right],
$$

where $C_{d}>0$ is a constant depending only on dimension $d$, it follows from (2.35) that if $\varphi \in \mathcal{H}(\Omega)$ satisfies $\varphi=\partial_{\xi} \psi$ for some $\psi \in L^{2}(\Omega)$, then $T_{\alpha, 0, \xi} \varphi \in \mathcal{H}(\Omega)$. One can also see further in this case that

$$
\lim _{\eta \rightarrow 0}\left\|T_{\alpha, \eta, \xi} \varphi-T_{\alpha, 0, \xi} \varphi\right\|=0 .
$$

Since $T_{\alpha, \eta, \xi} \varphi=0$ if $\partial_{\xi}^{*} \varphi=0$ for $\operatorname{Re}(\eta)>0$, it follows that 2.37) holds for $\varphi \in \mathcal{H}^{\prime}(\Omega)$, where $\mathcal{H}^{\prime}(\Omega)$ is the linear span of functions $\partial_{\xi} \psi, \psi \in L^{2}(\Omega)$ and $\varphi \in \mathcal{H}(\Omega)$ with $\partial_{\xi}^{*} \varphi=0$. Since $\left\|T_{\alpha, \eta, \xi}\right\| \leq 1, \operatorname{Re}(\eta)>0$, the norm of the operator $T_{\alpha, 0, \xi}: \mathcal{H}^{\prime}(\Omega) \rightarrow \mathcal{H}(\Omega)$ satisfies $\left\|T_{\alpha, 0, \xi}\right\| \leq 1$. Observe now that $\mathcal{H}^{\prime}(\Omega)$ is dense 
in $\mathcal{H}(\Omega)$. We conclude that $T_{\alpha, 0, \xi}$ extends to an operator on $\mathcal{H}(\Omega)$ with norm $\left\|T_{\alpha, 0, \xi}\right\| \leq 1$ and (2.37) holds for all $\varphi \in \mathcal{H}(\Omega)$.

We consider $\Psi(\xi, \eta, \cdot)$ defined by (2.31) for $\operatorname{Re}(\eta) \geq 0$. Since $\left\|T_{\Lambda, \eta, \xi}\right\| \leq 1$ for $\operatorname{Re}(\eta) \geq 0$, it follows that $\Psi(\xi, \eta, \cdot) \in L^{2}(\Omega, \mathcal{M}), \operatorname{Re}(\eta) \geq 0$. We conclude from (2.37) that

$$
\lim _{\eta \rightarrow 0}\|\Psi(\xi, \eta, \cdot)-\Psi(\xi, 0, \cdot)\|=0 .
$$

Hence from (2.24) $\lim _{\eta \rightarrow 0} q(\xi, \eta)=q(\xi, 0)$ exists for all $\xi \in[-\pi, \pi]^{d}$.

Observe from (2.31) the continuity of $q(\xi, 0)$ at $\xi=0$ will follow if we can show that for any $\varphi \in \mathcal{H}(\Omega)$ with $\langle\varphi\rangle=0$, and any $\varepsilon>0$, there exists $\delta>0$ such that $|\xi|<\delta$ implies

$$
\left\|T_{\alpha, \eta, \xi} \varphi-T_{\alpha, \eta, 0} \varphi\right\|<\varepsilon, \quad \operatorname{Re}(\eta) \geq 0 .
$$

To prove (2.38) consider first the case when $\varphi=\partial_{k, 0}^{*} \psi, \psi \in \mathcal{H}(\Omega)$. Then from (2.30) one has

$$
\begin{aligned}
& T_{\alpha, \eta, \xi} \varphi(\omega)=-e_{k}(-\xi) T_{\alpha, \eta, \xi} \psi(\omega) \\
& \quad+\alpha \exp \left[-i \mathbf{e}_{k} \cdot \xi\right] \sum_{t=0}^{\infty} e^{-\eta(t+1)} \sum_{x \in Z^{d}} \nabla_{k} \nabla \nabla^{*} G_{\alpha}(x, t) e^{-i x \cdot \xi} \psi\left(\tau_{x,-t-1} \omega\right), \quad \omega \in \Omega .
\end{aligned}
$$

Since $\left|\nabla_{k} \nabla \nabla^{*} G_{\alpha}(x, t)\right|$ is bounded by the RHS of (2.36) and $\left\|T_{\alpha, \eta, \xi}\right\| \leq 1$ one can find $\delta>0$ such that $|\xi|<\delta$ implies (2.38). Note now that if the operator $\tau_{\mathbf{e}_{k, 0}}$ is ergodic on $\Omega$, then the linear space $\left\{\partial_{k, 0}^{*} \psi: \psi \in \mathcal{H}(\Omega)\right\}$ is dense in $P[\mathcal{H}(\Omega)]$. Thus (2.38) holds for all $\varphi \in \mathcal{H}(\Omega)$ satisfying $\langle\varphi\rangle=0$. One can similarly prove (2.38) under the assumption that the operator $\tau_{0,1}$ is ergodic on $\Omega$.

Remark 3. The proof of the continuity of $q(\xi, 0)$ at $\xi=0$ given above is closely related to questions of proving the homogenized limit for (1.2). In fact, one expects the homogenized limiting equation to be a parabolic equation with constant coefficients given by $q(0,0)$. A proof of homogenization for equation (1.9) has been given in [8] using different techniques than those given here.

Let $q^{*}(\xi, \eta)$ be the Hermitian conjugate of the matrix $q(\xi, \eta)$ defined by (2.13) or (2.24). It was shown in [4] that if the operator $\tau_{0,1}=$ identity, then

$$
q^{*}(\xi, \eta)=q(\xi, \bar{\eta}), \quad \xi \in[-\pi, \pi]^{d}, \quad \operatorname{Re}(\eta)>0 .
$$

The identity (2.39) appears not to hold for the general situation here as we see in the following lemma.

Lemma 2.6. The identity (2.39) holds provided $\tau_{0,2}=$ identity.

Proof. Recall that the solution $\Phi(\xi, \eta, \omega)$ of (2.11) is a row vector. Let $\Phi^{*}(\xi, \eta, \omega)$ be the column vector whose entries are the complex conjugates of the row vector $\Phi(\xi, \eta, \omega)$. Then from (2.13) one has

$$
q^{*}(\xi, \eta)=\langle\mathbf{a}(\cdot)\rangle+\left\langle\Phi^{*}(\xi, \eta, \cdot) \partial_{\xi}^{*} \mathbf{a}(\cdot)\right\rangle .
$$


Observe now from (2.11) that

$$
\begin{aligned}
& \left\langle\Phi^{*}(\xi, \eta, \cdot) \partial_{\xi}^{*} \mathbf{a}(\cdot)\right\rangle=\left\langle\Phi^{*}(\xi, \eta, \cdot) P \partial_{\xi}^{*} \mathbf{a}(\cdot)\right\rangle \\
= & -\left\langle\Phi ^ { * } ( \xi , \eta , \cdot ) \left\{ e^{\bar{\eta}} \Phi\left(\xi, \bar{\eta}, \tau_{0,1} \cdot\right)-\Phi(\xi, \bar{\eta}, \cdot)\right.\right. \\
+ & \left.\left.P \partial_{\xi}^{*} \mathbf{a}(\cdot) \partial_{\xi} \Phi(\xi, \bar{\eta}, \cdot)\right\}\right\rangle \\
= & -\left\langle\left\{ e^{\eta} \Phi\left(\xi, \eta, \tau_{0,-1} \cdot\right)-\Phi(\xi, \eta, \cdot)\right.\right. \\
+ & \left.\left.P \partial_{\xi}^{*} \mathbf{a}(\cdot) \partial_{\xi} \Phi(\xi, \eta, \cdot)\right\}^{*} \Phi(\xi, \bar{\eta}, \cdot)\right\rangle .
\end{aligned}
$$

If we use now the assumption $\tau_{0,-1}=\tau_{0,1}$ the last identity implies

$$
\begin{aligned}
\left\langle\Phi^{*}(\xi, \eta, \cdot) \partial_{\xi}^{*} \mathbf{a}(\cdot)\right\rangle & =\left\langle\left\{\partial_{\xi}^{*} \mathbf{a}(\cdot)\right\}^{*} \Phi(\xi, \bar{\eta}, \cdot)\right\rangle \\
=\left\langle\mathbf{a}(\cdot) \partial_{\xi} \Phi(\xi, \bar{\eta}, \cdot)\right\rangle & =q(\xi, \bar{\eta})-\langle\mathbf{a}(\cdot)\rangle .
\end{aligned}
$$

Hence (2.39) follows.

We return now to the problem of obtaining sharper estimates on $\hat{G}_{\mathbf{a}}(\xi, \eta)$ than that given in Lemma 2.1. To accomplish this we shall obtain estimates on $q(\xi, \eta)$, which by Lemma 2.2 is defined for $\xi \in[-\pi, \pi]^{d}, \operatorname{Re}(\eta)>0,2 d \Lambda \leq 1$.

Lemma 2.7. Suppose $\xi \in[-\pi, \pi]^{d}, \quad \operatorname{Re}(\eta)>0,2 d \Lambda \leq 1$ and $q(\xi, \eta)$ is given by (2.13). Then for any column vector $\rho \in \mathbf{C}^{d}$ with adjoint $\rho^{*} \in \mathbf{C}^{d}$ there are the inequalities

$$
\begin{gathered}
0 \leq \operatorname{Re}\left[\rho^{*} q(\xi, \eta) \rho\right] \leq \Lambda|\rho|^{2}, \\
\left|\operatorname{Im}\left[\rho^{*} q(\xi, \eta) \rho\right]\right| \leq \Lambda|\rho|^{2} .
\end{gathered}
$$

Proof. Observe that since the solution $\Phi(\xi, \eta, \omega)$ of (2.11) is a row vector, the function $\varphi(\xi, \eta, \omega)=\Phi(\xi, \eta, \omega) \rho$ is a scalar. From (2.11) $\varphi(\xi, \eta, \omega)$ satisfies the equation

$$
e^{\eta} \varphi\left(\xi, \eta, \tau_{0,1} \omega\right)-\varphi(\xi, \eta, \omega)+P \partial_{\xi}^{*} \mathbf{a}(\omega) \partial_{\xi} \varphi(\xi, \eta, \omega)+P \partial_{\xi}^{*} \mathbf{a}(\omega) \rho=0 .
$$

From (2.13) we also have that

$$
\rho^{*} q(\xi, \eta) \rho=\rho^{*}\langle\mathbf{a}(\cdot)\rangle \rho+\left\langle\left[\overline{\partial_{\xi}^{*} \mathbf{a}(\cdot) \rho}\right] \varphi(\xi, \eta, \cdot)\right\rangle .
$$

Let $\theta=\operatorname{Im}(\eta)$, multiply (2.42) by $e^{-i \theta} \overline{\varphi\left(\xi, \eta, \tau_{0,1} \omega\right)}$ and take the expectation value. This yields

$$
\begin{aligned}
& -\left\langle\left[\partial_{\xi}^{*} \mathbf{a}(\cdot) \rho\right] e^{-i \theta} \overline{\varphi\left(\xi, \eta, \tau_{0,1} \cdot\right)}\right\rangle \\
& =e^{\operatorname{Re}(\eta)}\left\langle|\varphi(\xi, \eta, \cdot)|^{2}\right\rangle-\left\langle\varphi(\xi, \eta, \cdot) e^{-i \theta} \overline{\varphi\left(\xi, \eta, \tau_{0,1} \cdot\right)}\right\rangle \\
& +\left\langle e^{-i \theta} \overline{\varphi\left(\xi, \eta, \tau_{0,1} \cdot\right)} \partial_{\xi}^{*} \mathbf{a}(\cdot) \partial_{\xi} \varphi(\xi, \eta, \cdot)\right\rangle .
\end{aligned}
$$


If we take the real part of the previous equation we have

$$
\begin{aligned}
& -\operatorname{Re}\left\langle\left[\partial_{\xi}^{*} \mathbf{a}(\cdot) \rho\right] e^{-i \theta} \overline{\varphi\left(\xi, \eta, \tau_{0,1} \cdot\right)}\right\rangle \\
& =\left[e^{\operatorname{Re}(\eta)}-1\right]\left\langle|\varphi(\xi, \eta, \cdot)|^{2}\right\rangle \\
& +\frac{1}{2}\left\langle\left|\varphi(\xi, \eta, \cdot)-e^{i \theta} \varphi\left(\xi, \eta, \tau_{0,1} \cdot\right)\right|^{2}\right\rangle \\
& +\operatorname{Re}\left\langle e^{-i \theta} \overline{\varphi\left(\xi, \eta, \tau_{0,1} \cdot\right)} \partial_{\xi}^{*} \mathbf{a}(\cdot) \partial_{\xi} \varphi(\xi, \eta, \cdot)\right\rangle .
\end{aligned}
$$

Since $2 d \Lambda \leq 1$, there are positive numbers $A$ and $B$ satisfying

$$
\begin{aligned}
A^{2}= & \frac{1}{4}\left\langle\left[\overline{\varphi(\xi, \eta, \cdot)+e^{i \theta} \varphi\left(\xi, \eta, \tau_{0,1} \cdot\right)}\right]\right. \\
B^{2}= & \frac{1}{4}\left\langle\left[\overline{\varphi\left(\xi, \eta(\cdot) \partial_{\xi}\left[\varphi(\xi, \eta, \cdot)+e^{i \theta} \varphi\left(\xi, \eta, \tau_{0,1} \cdot\right)\right.\right.}\right]\right. \\
& {\left.\left[2-\partial_{\xi}^{*} \mathbf{a}(\cdot) \partial_{\xi}\right]\left[\varphi(\xi, \eta, \cdot)-e^{i \theta} \varphi\left(\xi, \eta, \tau_{0,1} \cdot\right)\right]\right\rangle . }
\end{aligned}
$$

We see from (2.44) that there is the identity

$$
\begin{aligned}
& -\operatorname{Re}\left\langle\left[\partial_{\xi}^{*} \mathbf{a}(\cdot) \rho\right] e^{-i \theta} \overline{\varphi\left(\xi, \eta, \tau_{0,1} \cdot\right)}\right\rangle \\
& =\left[e^{\operatorname{Re}(\eta)}-1\right]\left\langle|\varphi(\xi, \eta, \cdot)|^{2}\right\rangle+A^{2}+B^{2} .
\end{aligned}
$$

Observe now that one has

$$
\begin{aligned}
& -\left\langle\left[\partial_{\xi}^{*} \mathbf{a}(\cdot) \rho\right] e^{-i \theta} \overline{\varphi\left(\xi, \eta, \tau_{0,1} \cdot\right)}\right\rangle \\
& =\frac{1}{2}\left\langle\left[\partial_{\xi}^{*} \mathbf{a}(\cdot) \rho\right]\left[\overline{\varphi(\xi, \eta, \cdot)-e^{i \theta} \varphi\left(\xi, \eta, \tau_{0,1} \cdot\right)}\right]\right\rangle \\
& -\frac{1}{2}\left\langle\left[\partial_{\xi}^{*} \mathbf{a}(\cdot) \rho\right]\left[\overline{\varphi(\xi, \eta, \cdot)+e^{i \theta} \varphi\left(\xi, \eta, \tau_{0,1} \cdot\right)}\right]\right\rangle .
\end{aligned}
$$

It is clear from the Schwarz inequality that

$$
\begin{aligned}
& \left|\frac{1}{2}\left\langle\left[\partial_{\xi}^{*} \mathbf{a}(\cdot) \rho\right]\left[\overline{\varphi(\xi, \eta, \cdot)+e^{i \theta} \varphi\left(\xi, \eta, \tau_{0,1} \cdot\right)}\right]\right\rangle\right| \\
\leq & \left\langle\rho^{*} \mathbf{a}(\cdot) \rho\right\rangle^{1 / 2} A .
\end{aligned}
$$

Next observe that

$$
\begin{aligned}
& \frac{1}{2}\left\langle\left[\partial_{\xi}^{*} \mathbf{a}(\cdot) \rho\right]\left[\overline{\varphi(\xi, \eta, \cdot)-e^{i \theta} \varphi\left(\xi, \eta, \tau_{0,1} \cdot\right)}\right]\right\rangle \\
= & -\frac{1}{2}\left\langle\rho^{*}\left[\Lambda I_{d}-\mathbf{a}(\cdot)\right]\left[\overline{\partial_{\xi} \varphi(\xi, \eta, \cdot)-e^{i \theta} \partial_{\xi} \varphi\left(\xi, \eta, \tau_{0,1} \cdot\right)}\right]\right\rangle .
\end{aligned}
$$

Since $2 d \Lambda \leq 1$, we also have that

$$
\begin{aligned}
& \frac{1}{4}\left\langle\left[\left[\overline{\partial_{\xi} \varphi(\xi, \eta, \cdot)-e^{i \theta} \partial_{\xi} \varphi\left(\xi, \eta, \tau_{0,1} \cdot\right)}\right]\right.\right. \\
& \left.\quad\left[\Lambda I_{d}-\mathbf{a}(\cdot)\right]\left[\partial_{\xi} \varphi(\xi, \eta, \cdot)-e^{i \theta} \partial_{\xi} \varphi\left(\xi, \eta, \tau_{0,1} \cdot\right)\right]\right\rangle \leq B^{2} .
\end{aligned}
$$

We conclude then from the last two inequalities that

$$
\begin{aligned}
& \left|\frac{1}{2}\left\langle\left[\partial_{\xi}^{*} \mathbf{a}(\cdot) \rho\right]\left[\overline{\varphi(\xi, \eta, \cdot)-e^{i \theta} \varphi\left(\xi, \eta, \tau_{0,1} \cdot\right)}\right]\right\rangle\right| \\
\leq & \left\langle\rho^{*}\left[\Lambda I_{d}-\mathbf{a}(\cdot)\right] \rho\right\rangle^{1 / 2} B .
\end{aligned}
$$


The inequalities (2.45), (2.46), (2.47), (2.48) now imply the inequality

$$
A^{2}+B^{2} \leq\left\langle\rho^{*} \mathbf{a}(\cdot) \rho\right\rangle^{1 / 2} A+\left\langle\rho^{*}\left[\Lambda I_{d}-\mathbf{a}(\cdot)\right] \rho\right\rangle^{1 / 2} B
$$

If we apply the Schwarz inequality to this last inequality, we have

$$
A^{2}+B^{2} \leq \Lambda|\rho|^{2}
$$

Observe now that from $(2.43)$,

$$
\begin{aligned}
& \rho^{*} q(\xi, \eta) \rho-\rho^{*}\langle\mathbf{a}(\cdot)\rangle \rho \\
= & \frac{1}{2}\left\langle\left[\overline{\partial_{\xi}^{*} \mathbf{a}(\cdot) \rho}\right]\left[\varphi(\xi, \eta, \cdot)+e^{i \theta} \varphi\left(\xi, \eta, \tau_{0,1} \cdot\right)\right]\right\rangle \\
+ & \left.\frac{1}{2}\left\langle\overline{\left[\partial_{\xi}^{*} \mathbf{a}(\cdot) \rho\right.}\right]\left[\varphi(\xi, \eta, \cdot)-e^{i \theta} \varphi\left(\xi, \eta, \tau_{0,1} \cdot\right)\right]\right\rangle .
\end{aligned}
$$

It follows from this last identity and (2.47), 2.48), 2.49 that

$$
\left|\rho^{*} q(\xi, \eta) \rho-\rho^{*}\langle\mathbf{a}(\cdot)\rangle \rho\right| \leq \Lambda|\rho|^{2} .
$$

The inequality 2.41 clearly follows from this last inequality.

We turn to the proof of (2.40). We note from (2.43) that one has

$$
\begin{aligned}
\rho^{*} q(\xi, \eta) \rho & =\rho^{*}\langle\mathbf{a}(\cdot)\rangle \rho+\left\langle\left[\overline{\partial_{\xi}^{*} \mathbf{a}(\cdot) \rho}\right] e^{i \theta} \varphi\left(\xi, \eta, \tau_{0,1} \cdot\right)\right\rangle \\
& \left.+\left\langle\overline{\partial_{\xi}^{*} \mathbf{a}(\cdot) \rho}\right]\left[\varphi(\xi, \eta, \cdot)-e^{i \theta} \varphi\left(\xi, \eta, \tau_{0,1} \cdot\right)\right]\right\rangle .
\end{aligned}
$$

Hence from (2.45), (2.48) it follows that

$$
\begin{aligned}
\operatorname{Re}\left[\rho^{*} q(\xi, \eta) \rho\right] & \leq \rho^{*}\langle\mathbf{a}(\cdot)\rangle \rho-A^{2}-B^{2} \\
+2\left\langle\rho^{*}\left[\Lambda I_{d}-\mathbf{a}(\cdot)\right] \rho\right\rangle^{1 / 2} B & \leq \Lambda|\rho|^{2}-A^{2} \leq \Lambda|\rho|^{2} .
\end{aligned}
$$

To obtain the lower bound in (2.40) we write

$$
\begin{aligned}
\rho^{*} q(\xi, \eta) \rho & =\rho^{*}\langle\mathbf{a}(\cdot)\rangle \rho-\left\langle\left[\overline{\partial_{\xi}^{*} \mathbf{a}(\cdot) \rho}\right] e^{i \theta} \varphi\left(\xi, \eta, \tau_{0,1} \cdot\right)\right\rangle \\
& \left.+\left\langle\overline{\partial_{\xi}^{*} \mathbf{a}(\cdot) \rho}\right]\left[\varphi(\xi, \eta, \cdot)+e^{i \theta} \varphi\left(\xi, \eta, \tau_{0,1} \cdot\right)\right]\right\rangle
\end{aligned}
$$

Hence from (2.45), 2.47) it follows that

$$
\begin{aligned}
\operatorname{Re}\left[\rho^{*} q(\xi, \eta) \rho\right] & \geq \rho^{*}\langle\mathbf{a}(\cdot)\rangle \rho+A^{2}+B^{2} \\
-2\left\langle\rho^{*} \mathbf{a}(\cdot) \rho\right\rangle^{1 / 2} A & \geq B^{2} \geq 0 .
\end{aligned}
$$

This last inequality completes the proof of (2.40).

Lemma 2.8. Suppose $\xi \in[-\pi, \pi]^{d}, 2 d \Lambda \leq 1, \operatorname{Re}(\eta)>0$ and $\theta=\operatorname{Im}(\eta)$. If $q(\xi, \eta)$ is given by (2.13) and $\rho \in \mathbf{C}^{d}$ is a column vector with adjoint $\rho^{*} \in \mathbf{C}^{d}$, then there is the inequality,

$$
\operatorname{Re}\left[e^{-i \theta} \rho^{*} q(\xi, \eta) \rho\right] \geq \frac{1}{2}(1+\cos \theta) \lambda|\rho|^{2}-\frac{1}{2}(1-\cos \theta) \Lambda|\rho|^{2} .
$$


Proof. We first prove the inequality assuming $\lambda=0$. To see this note the identity

$$
\begin{aligned}
& \operatorname{Re}\left[e^{-i \theta}\left\langle\left[\overline{\partial_{\xi}^{*} \mathbf{a}(\cdot) \rho}\right] \varphi(\xi, \eta, \cdot)\right\rangle\right] \\
=- & \operatorname{Re}\left\langle\left[\overline{\partial_{\xi}^{*} \mathbf{a}(\cdot) \rho}\right] e^{i \theta} \varphi\left(\xi, \eta, \tau_{0,1} \cdot\right)\right\rangle \\
+ & \operatorname{Re}\left\{\left(\frac{1+e^{-i \theta}}{2}\right)\left\langle\left[\overline{\partial_{\xi}^{*} \mathbf{a}(\cdot) \rho}\right]\left[\varphi(\xi, \eta, \cdot)+e^{i \theta} \varphi\left(\xi, \eta, \tau_{0,1} \cdot\right)\right]\right\rangle\right\} \\
- & \operatorname{Re}\left\{\left(\frac{1-e^{-i \theta}}{2}\right)\left\langle\left[\overline{\partial_{\xi}^{*} \mathbf{a}(\cdot) \rho}\right]\left[\varphi(\xi, \eta, \cdot)-e^{i \theta} \varphi\left(\xi, \eta, \tau_{0,1} \cdot\right)\right]\right\rangle\right\} .
\end{aligned}
$$

On using the inequalities (2.45), (2.47), (2.48) we have from the last identity,

$$
\begin{aligned}
\operatorname{Re}\left[e^{-i \theta} \rho^{*} q(\xi, \eta) \rho\right] & \geq \cos \theta\left\langle\rho^{*} \mathbf{a}(\cdot) \rho\right\rangle \\
+A^{2}+B^{2} & -2|\cos (\theta / 2)|\left\langle\rho^{*} \mathbf{a}(\cdot) \rho\right\rangle^{1 / 2} A \\
& -2|\sin (\theta / 2)|\left\langle\rho^{*}\left[\Lambda I_{d}-\mathbf{a}(\cdot)\right] \rho\right\rangle^{1 / 2} B .
\end{aligned}
$$

On using the Schwarz inequality we conclude from the last inequality that

$$
\operatorname{Re}\left[e^{-i \theta} \rho^{*} q(\xi, \eta) \rho\right] \geq-\Lambda|\rho|^{2} \sin ^{2}(\theta / 2),
$$

which is the same as (2.50) with $\lambda=0$.

To get 2.50) for $\lambda>0$, observe that

$$
\begin{aligned}
\cos ^{2}(\theta / 2)\left\langle\rho^{*} \mathbf{a}(\cdot) \rho\right\rangle-\operatorname{Re}\left\langle\left[\overline{\partial_{\xi}^{*} \mathbf{a}(\cdot) \rho}\right] e^{i \theta} \varphi\left(\xi, \eta, \tau_{0,1} \cdot\right)\right\rangle \\
+\operatorname{Re}\left\{\left(\frac{1+e^{-i \theta}}{2}\right)\left\langle\left[\overline{\partial_{\xi}^{*} \mathbf{a}(\cdot) \rho}\right]\left[\varphi(\xi, \eta, \cdot)+e^{i \theta} \varphi\left(\xi, \eta, \tau_{0,1} \cdot\right)\right]\right\rangle\right\} \\
=\left[e^{\operatorname{Re}(\eta)}-1\right]\left\langle|\varphi(\xi, \eta, \cdot)|^{2}\right\rangle+B^{2} \\
\left.+\left\langle\overline{\cos (\theta / 2) e^{i \theta / 2} \rho+\frac{1}{2} \partial_{\xi} \varphi(\xi, \eta, \cdot)+\frac{1}{2} e^{i \theta} \partial_{\xi} \varphi\left(\xi, \eta, \tau_{0,1} \cdot\right)}\right]\right\rangle \\
\left.\quad \mathbf{a}(\cdot)\left[\cos (\theta / 2) e^{i \theta / 2} \rho+\frac{1}{2} \partial_{\xi} \varphi(\xi, \eta, \cdot)+\frac{1}{2} e^{i \theta} \varphi\left(\xi, \eta, \tau_{0,1} \cdot\right)\right]\right\rangle .
\end{aligned}
$$

Since $\mathbf{a}(\cdot) \geq \lambda I_{d}$, the quantity on the RHS of the last equation is bounded below by $\lambda|\rho|^{2} \cos ^{2}(\theta / 2)+B^{2}$. Proceeding now as we did to prove (2.51) we obtain (2.50).

Corollary 2.1. Suppose $\xi \in[-\pi, \pi]^{d}, 2 d \Lambda \leq 1, \operatorname{Re}(\eta)>0$ and $\theta=\operatorname{Im}(\eta)$. If $q(\xi, \eta)$ is given by (2.13) and $\rho \in \mathbf{C}^{d}$ is a column vector with adjoint $\rho^{*} \in \mathbf{C}^{d}$, then there is the inequality

$$
\left|e^{\eta}-1+\rho^{*} q(\xi, \eta) \rho\right| \geq e^{R e(\eta)}-1+\frac{1}{2}(1+\cos \theta) \lambda|\rho|^{2}+\frac{1}{2}(1-\cos \theta)\left(2-\Lambda|\rho|^{2}\right) .
$$

Proof. The result follows from Lemma 2.8 by observing that

$$
\left|e^{\eta}-1+\rho^{*} q(\xi, \eta) \rho\right| \geq e^{\operatorname{Re}(\eta)}-\cos \theta+\operatorname{Re}\left[e^{-i \theta} \rho^{*} q(\xi, \eta) \rho\right] .
$$

Remark 4. Observe that Corollary 2.1 gives an improvement on Lemma 2.1 by taking $\rho=e(-\xi)$ in (2.52) and using the formula (2.12). 
If the matrix $\mathbf{a}(\omega)$ of (1.1) generates a Markov chain in (1.2) for all $\omega \in \Omega$, then the function $G_{\mathbf{a}}(x, t)$ is nonnegative for $x \in \mathbf{Z}^{d}, t \in \mathbf{Z}, t \geq 0$. From (1.7) it then follows that the function $\hat{G}_{\mathbf{a}}(\xi, t)$ defined by

$$
\hat{G}_{\mathbf{a}}(\xi, t)=\sum_{x \in \mathbf{Z}^{d}} G_{\mathbf{a}}(x, t) e^{i x \cdot \xi}, \quad \xi \in[-\pi, \pi]^{d},
$$

satisfies $\left|\hat{G}_{\mathbf{a}}(\xi, t)\right| \leq 1$. We wish to show now that the inequalities obtained in Lemmas $2.7,2.8$ imply that $\left|\hat{G}_{\mathbf{a}}(\xi, t)\right|$ is bounded by $C(\Lambda / \lambda)$ for some universal constant $C$. To prove this we first observe from (2.1) that

$$
\begin{aligned}
\hat{G}_{\mathbf{a}}(\xi, t) & =\frac{1}{2 \pi} \int_{-\pi}^{\pi} \hat{G}_{\mathbf{a}}(\xi, \eta) e^{\eta t} d[\operatorname{Im}(\eta)] \\
& =\frac{e^{\operatorname{Re}(\eta) t}}{2 \pi} \int_{0}^{\pi}\left[\hat{G}_{\mathbf{a}}(\xi, \eta)+\hat{G}_{\mathbf{a}}(\xi, \bar{\eta})\right] \cos [\operatorname{Im}(\eta) t] d[\operatorname{Im}(\eta)] \\
& +\frac{i e^{\operatorname{Re}(\eta) t}}{2 \pi} \int_{0}^{\pi}\left[\hat{G}_{\mathbf{a}}(\xi, \eta)-\hat{G}_{\mathbf{a}}(\xi, \bar{\eta})\right] \sin [\operatorname{Im}(\eta) t] d[\operatorname{Im}(\eta)],
\end{aligned}
$$

where the integration with respect to $\operatorname{Im}(\eta)$ is for fixed $\operatorname{Re}(\eta)>0$.

Lemma 2.9. Suppose $\xi \in[-\pi, \pi]^{d}, 4 d \Lambda \leq 1, \operatorname{Re}(\eta)>0$. Then there is a universal constant $C$ such that

$$
\int_{0}^{\pi}\left|\hat{G}_{\mathbf{a}}(\xi, \eta)+\hat{G}_{\mathbf{a}}(\xi, \bar{\eta})\right| d[\operatorname{Im}(\eta)] \leq C\left(\frac{\Lambda}{\lambda}\right) .
$$

Proof. By Lemma 2.1 it is sufficient to show

$$
e^{-\operatorname{Re}(\eta)} \int_{0}^{\pi}\left|\hat{G}_{\mathbf{a}}(\xi, \eta)+\hat{G}_{\mathbf{a}}(\xi, \bar{\eta})\right| d[\operatorname{Im}(\eta)] \leq C\left(\frac{\Lambda}{\lambda}\right) .
$$

From (2.52) we have if $\pi / 2 \leq|\operatorname{Im}(\eta)| \leq \pi$, then

$$
\left|e^{\eta}-1+e(\xi) q(\xi, \eta) e(-\xi)\right| \geq 1 / 2,
$$

whence (2.12) implies

$$
e^{-\operatorname{Re}(\eta)} \int_{\pi / 2}^{\pi}\left|\hat{G}_{\mathbf{a}}(\xi, \eta)+\hat{G}_{\mathbf{a}}(\xi, \bar{\eta})\right| d[\operatorname{Im}(\eta)] \leq 2 \pi .
$$

Next we define $\alpha>0$ to be

$$
\alpha=\min \left\{\frac{\pi}{2}, e^{\operatorname{Re}(\eta)}-1+\pi \Lambda|e(\xi)|^{2}\right\} .
$$

It follows again from (2.52) that

$$
e^{-\operatorname{Re}(\eta)} \int_{0}^{\alpha}\left|\hat{G}_{\mathbf{a}}(\xi, \eta)+\hat{G}_{\mathbf{a}}(\xi, \bar{\eta})\right| d[\operatorname{Im}(\eta)] \leq 4 \pi\left(\frac{\Lambda}{\lambda}\right) .
$$

Next we consider $\operatorname{Im}(\eta)$ in the interval $\alpha \leq \operatorname{Im}(\eta) \leq \pi / 2$. Observe from Lemma 2.7 that we have the inequality

$$
\begin{aligned}
\left|e^{\eta}-1+e(\xi) q(\xi, \eta) e(-\xi)\right| & \geq \sin [\operatorname{Im}(\eta)] \\
+\operatorname{Im}\left[e^{-i \operatorname{Im}(\eta)} e(\xi) q(\xi, \eta) e(-\xi)\right] & \geq \frac{2}{\pi} \operatorname{Im}(\eta)-\Lambda \sqrt{2}|e(\xi)|^{2} \\
& \geq \frac{(2-\sqrt{2})}{\pi} \operatorname{Im}(\eta) .
\end{aligned}
$$


We also have from Lemma 2.7 that

$$
\begin{aligned}
& \left|e^{\eta}+e^{\bar{\eta}}-2+e(\xi) q(\xi, \eta) e(-\xi)+e(\xi) q(\xi, \bar{\eta}) e(-\xi)\right| \\
\leq & 2\left[e^{\operatorname{Re}(\eta)}-1+\sqrt{2} \Lambda|e(\xi)|^{2}+1-\cos [\operatorname{Im}(\eta)]\right] .
\end{aligned}
$$

We conclude from these last two inequalities that

Hence,

$$
e^{-\operatorname{Re}(\eta)}\left|\hat{G}_{\mathbf{a}}(\xi, \eta)+\hat{G}_{\mathbf{a}}(\xi, \bar{\eta})\right| \leq \frac{2 \pi^{2} \alpha}{(2-\sqrt{2})^{2}[\operatorname{Im}(\eta)]^{2}}+\frac{\pi^{2}}{(2-\sqrt{2})^{2}} .
$$

$$
e^{-\operatorname{Re}(\eta)} \int_{\alpha}^{\pi / 2}\left|\hat{G}_{\mathbf{a}}(\xi, \eta)+\hat{G}_{\mathbf{a}}(\xi, \bar{\eta})\right| d[\operatorname{Im}(\eta)] \leq C,
$$

for some universal constant $C$.

Lemma 2.10. Suppose $\xi \in[-\pi, \pi]^{d}, 4 d \Lambda \leq 1, \operatorname{Re}(\eta)>0$. Then there is a universal constant $C>0$ such that for any $t>0$,

$$
\left|\int_{0}^{\pi} \hat{G}_{\mathbf{a}}(\xi, \eta) \sin [\operatorname{Im}(\eta) t] d[\operatorname{Im}(\eta)]\right| \leq C\left(\frac{\Lambda}{\lambda}\right) .
$$

Proof. By the arguments of Lemma 2.9 it will be sufficient for us to show that there is a universal constant $C$ such that

$$
e^{-\operatorname{Re}(\eta)}\left|\int_{\alpha}^{\pi / 2} \hat{G}_{\mathbf{a}}(\xi, \eta) \sin [\operatorname{Im}(\eta) t] d[\operatorname{Im}(\eta)]\right| \leq C .
$$

Note that if $0<t<8$, the inequality (2.55) follows from 2.54). Hence we can assume $t \geq 8$. Observe also that if $\alpha \geq \pi / 4$, then 2.55) follows from 2.52). Thus we are left to prove (2.55) when $t \geq 8, \alpha<\pi / 4$. In that case we write

$$
\begin{aligned}
& e^{-\operatorname{Re}(\eta)} \int_{\alpha}^{\pi / 2} \hat{G}_{\mathbf{a}}(\xi, \eta) \sin [\operatorname{Im}(\eta) t] d[\operatorname{Im}(\eta)] \\
= & \frac{1}{2} e^{-\operatorname{Re}(\eta)} \int_{\alpha}^{\pi / 2-\pi / t}\left[\hat{G}_{\mathbf{a}}(\xi, \eta)-\hat{G}_{\mathbf{a}}\left(\xi, \eta+\frac{i \pi}{t}\right)\right] \sin [\operatorname{Im}(\eta) t] d[\operatorname{Im}(\eta)] \\
+ & \frac{1}{2} e^{-\operatorname{Re}(\eta)} \int_{\alpha}^{\alpha+\pi / t} \hat{G}_{\mathbf{a}}(\xi, \eta) \sin [\operatorname{Im}(\eta) t] d[\operatorname{Im}(\eta)] \\
+ & \frac{1}{2} e^{-\operatorname{Re}(\eta)} \int_{\pi / 2-\pi / t}^{\pi / 2} \hat{G}_{\mathbf{a}}(\xi, \eta) \sin [\operatorname{Im}(\eta) t] d[\operatorname{Im}(\eta)] .
\end{aligned}
$$

It follows from (2.54) that the last 2 integrals in the previous expression are bounded by a universal constant. To bound the first integral in the expression we use the identity

$$
\begin{aligned}
& e^{-\operatorname{Re}(\eta)} \hat{G}_{\mathbf{a}}(\xi, \eta)-e^{-\operatorname{Re}(\eta)} \hat{G}_{\mathbf{a}}\left(\xi, \eta+\frac{i \pi}{t}\right) \\
= & e^{-\operatorname{Re}(\eta)} \hat{G}_{\mathbf{a}}(\xi, \eta) e^{-\operatorname{Re}(\eta)} \hat{G}_{\mathbf{a}}\left(\xi, \eta+\frac{i \pi}{t}\right) \\
& \left\{e^{-i \operatorname{Im}(\eta)}\left[1-e^{-i \pi / t}\right]-e^{-i \operatorname{Im}(\eta)} e(\xi) q(\xi, \eta) e(-\xi)\right. \\
& \left.+e^{-i[\operatorname{Im}(\eta)+\pi / t]} e(\xi) q\left(\xi, \eta+\frac{i \pi}{t}\right) e(-\xi)\right\} .
\end{aligned}
$$


Hence from (2.54) and Lemma 2.7 there is a universal constant $C>0$ such that the first integral is bounded in absolute value by the expression

$$
\begin{aligned}
C \int_{\alpha}^{\alpha+\pi / t} & \frac{d[\operatorname{Im}(\eta)]}{[\operatorname{Im}(\eta)+\pi / t]}+\frac{C}{t} \int_{\alpha+\pi / t}^{\pi / 2-\pi / t} \frac{d[\operatorname{Im}(\eta)]}{\operatorname{Im}(\eta)[\operatorname{Im}(\eta)+\pi / t]} \\
+C \Lambda|e(\xi)|^{2} \int_{\alpha}^{\pi / 2-\pi / t} & \frac{d[\operatorname{Im}(\eta)]}{\operatorname{Im}(\eta)[\operatorname{Im}(\eta)+\pi / t]} .
\end{aligned}
$$

It is easy to see that the previous expression is bounded by a universal constant. We have proved (2.55) when $t \geq 8, \alpha<\pi / 4$.

From (2.53) and Lemmas $2.9,2.10$ we see, by letting $\operatorname{Re}(\eta) \rightarrow 0$, that there is a universal constant $C>0$ such that $\left|\hat{G}_{\mathbf{a}}(\xi, t)\right| \leq C(\Lambda / \lambda), \xi \in[-\pi, \pi]^{d}, t \in \mathbf{Z}, t \geq 0$. We can also obtain a bound on the discrete derivative of $\hat{G}_{\mathbf{a}}(\xi, t)$ with respect to $t$.

Lemma 2.11. Suppose $\xi \in[-\pi, \pi]^{d}, 4 d \Lambda \leq 1, t \in \mathbf{Z}, t \geq 0$. Then for any $\delta$ satisfying $0 \leq \delta<1$ there is a constant $C_{\delta}>0$, depending only on $\delta$, such that the function $\hat{G}_{\mathbf{a}}(\xi, t)$ of (2.53) satisfies the inequality

$$
\left|\hat{G}_{\mathbf{a}}(\xi, t+1)-\hat{G}_{\mathbf{a}}(\xi, t)\right| \leq \frac{C_{\delta} \Lambda}{\lambda}\left[\Lambda|e(\xi)|^{2}\right]^{\delta} .
$$

Proof. From (2.12), (2.53) we have that

$$
\begin{aligned}
\hat{G}_{\mathbf{a}}(\xi, t+1)-\hat{G}_{\mathbf{a}}(\xi, t) & =\frac{1}{2 \pi} \int_{-\pi}^{\pi} \frac{e^{\eta(t+1)}\left(e^{\eta}-1\right)}{e^{\eta}-1+e(\xi) q(\xi, \eta) e(-\xi)} d[\operatorname{Im}(\eta)] \\
& =-\frac{1}{2 \pi} \int_{-\pi}^{\pi} \frac{e^{\eta(t+1)} e(\xi) q(\xi, \eta) e(-\xi)}{e^{\eta}-1+e(\xi) q(\xi, \eta) e(-\xi)} d[\operatorname{Im}(\eta)] \\
& =-\frac{1}{2 \pi} \int_{-\pi}^{\pi} e(\xi) q(\xi, \eta) e(-\xi) \hat{G}_{\mathbf{a}}(\xi, \eta) e^{\eta t} d[\operatorname{Im}(\eta)] .
\end{aligned}
$$

We have, therefore, from Lemma 2.7 that

$$
\left|\hat{G}_{\mathbf{a}}(\xi, t+1)-\hat{G}_{\mathbf{a}}(\xi, t)\right| \leq \frac{\sqrt{2} \Lambda|e(\xi)|^{2}}{2 \pi} e^{\operatorname{Re}(\eta) t} \int_{-\pi}^{\pi}\left|\hat{G}_{\mathbf{a}}(\xi, \eta)\right| d[\operatorname{Im}(\eta)] .
$$

We argue as in Lemma 2.9. Observe now from (2.54) we have the inequality

$$
\begin{gathered}
e^{-\operatorname{Re}(\eta)} \int_{\alpha}^{\pi / 2}\left|\hat{G}_{\mathbf{a}}(\xi, \eta)\right| d[\operatorname{Im}(\eta)] \\
\leq \frac{\pi}{2-\sqrt{2}} \int_{\alpha}^{\pi / 2} \frac{d[\operatorname{Im}(\eta)]}{\operatorname{Im}(\eta)}=\frac{\pi}{2-\sqrt{2}} \ln \left(\frac{\pi}{2 \alpha}\right) .
\end{gathered}
$$

The result follows from the previous inequality and the observation that $\Lambda|e(\xi)|^{2} \leq$ $4 d \Lambda \leq 1$, since we may bound the integral of $\left|\hat{G}_{\mathbf{a}}(\xi, \eta)\right|$ on the intervals $(0, \alpha)$ and $(\pi / 2, \pi)$ exactly as we did in Lemma 2.9 .

\section{Interpolation estimates}

Let $E$ be a Banach space with norm $\|\cdot\|$. For $1 \leq p \leq \infty$ we define the space $L^{p}\left([-\pi, \pi]^{d+1}, E\right)$ in the usual way. Thus if $p<\infty$, the space consists of all measurable functions $A:[-\pi, \pi]^{d+1} \rightarrow E$ with finite norm $\|A\|_{p}$, where

$$
\|A\|_{p}^{p}=\int_{[-\pi, \pi]^{d+1}}\|A(\zeta)\|^{p} d \zeta
$$


Correspondingly we define the $p=\infty$ norm $\|A\|_{\infty}$ by

$$
\|A\|_{\infty}=\sup _{\zeta \in[-\pi, \pi]^{d+1}}\|A(\zeta)\| .
$$

We shall also be interested in weak $L^{p}$ spaces. Thus for $1 \leq p<\infty$ we define the space $L_{w}^{p}\left([-\pi, \pi]^{d+1}, E\right)$ as all measurable functions $A:[-\pi, \pi]^{d+1} \rightarrow E$ with finite norm $\|A\|_{p, w}$, where

$$
\|A\|_{p, w}^{p}=\sup _{\alpha>0}\left[\alpha^{p} \text { meas }\left\{\zeta \in[-\pi, \pi]^{d+1}:\|A(\zeta)\|>\alpha\right\}\right] .
$$

Here we shall be interested in situations where $E$ is in fact a Hilbert space. In $\S 2$ we already introduced the space $\mathcal{M}$ of complex $d \times d$ matrices $A$ with adjoint $A^{*}$. Evidently we can make $\mathcal{M}$ into a Hilbert space by defining the norm of $A \in \mathcal{M}$ to be

$$
\|A\|^{2}=\operatorname{Tr}\left[A^{*} A\right] .
$$

In $\S 2$ we also introduced the Hilbert space $L^{2}(\Omega, \mathcal{M})$ of square integrable functions $A: \Omega \rightarrow \mathcal{M}$. In the following we shall be taking $E=\mathcal{M}$ and $E=L^{2}(\Omega, \mathcal{M})$.

For a function $A:[-\pi, \pi]^{d+1} \rightarrow \mathcal{M}$ we define its Fourier transform $\mathcal{F}(A)(y)=$ $\hat{A}(y), y \in Z^{d+1}$, by

$$
\hat{A}(y)=\frac{1}{(2 \pi)^{d+1}} \int_{[-\pi, \pi]^{d+1}} A(\zeta) e^{-i y \cdot \zeta} d \zeta .
$$

The Plancherel theorem then yields the identity

$$
\|A\|_{2}^{2}=(2 \pi)^{d+1} \sum_{y \in \mathbf{Z}^{d+1}}\|\hat{A}(y)\|^{2} .
$$

Similarly, one sees that for two functions $A, B:[-\pi, \pi]^{d+1} \rightarrow \mathcal{M}$, the Fourier transform of the product $A B(\zeta)=A(\zeta) B(\zeta), \zeta \in[-\pi, \pi]^{d+1}$, is given by

$$
\mathcal{F}(A B)(y)=\sum_{y^{\prime} \in \mathbf{Z}^{d+1}} \hat{A}\left(y^{\prime}\right) \hat{B}\left(y-y^{\prime}\right), \quad y \in \mathbf{Z}^{d+1} .
$$

We assume now that the symmetric $d \times d$ matrix $\mathbf{b}(\omega)$ satisfies (2.21) and also that $4 d \Lambda \leq 1$. Hence the operator $T_{\Lambda, \eta, \xi}$ of $(2.30)$ is bounded on $L^{2}(\Omega, \mathcal{M})$ with norm not exceeding 1 , provided $\operatorname{Re}(\eta)>0, \xi \in[-\pi, \pi]^{d}$. We define an operator $\mathcal{S}_{1}$ on functions $A:[-\pi, \pi]^{d+1} \rightarrow \mathcal{M}$ with the property that $\hat{A}$ has finite support in $\mathbf{Z}^{d+1}$. To do this we denote by $R$ the reflection $R: \mathbf{Z}^{d+1} \rightarrow \mathbf{Z}^{d+1}$ given by $R(x, t)=(x,-t), x \in \mathbf{Z}^{d}, t \in \mathbf{Z}$. For $\xi \in[-\pi, \pi]^{d}, \eta \in \mathbf{C}$ with $\operatorname{Re}(\eta)>0$ we put $\zeta=(\xi, \operatorname{Im}(\eta))$. Then $\mathcal{S}_{1}(A)$ is a mapping from $[-\pi, \pi]^{d+1}$ to $L^{2}(\Omega, \mathcal{M})$ given by

$$
\begin{aligned}
\mathcal{S}_{1}(A)(\zeta) & =\sum_{y \in \mathbf{Z}^{d+1}} \hat{A}(y) e^{-i y \cdot \zeta} \tau_{R y} P \mathbf{b} \sum_{m=0}^{\infty}\left(T_{\Lambda, \eta, \xi} P \mathbf{b}\right)^{m} \\
& \left.=\sum_{y \in \mathbf{Z}^{d+1}} \hat{A}(y) e^{-i y \cdot \zeta} \tau_{R y} P \mathbf{b}\left[I-T_{\Lambda, \eta, \xi} P \mathbf{b}\right)\right]^{-1} .
\end{aligned}
$$

In (3.3) the operator $\tau_{z}, z \in \mathbf{Z}^{d+1}$, on functions $g: \Omega \rightarrow \mathbf{C}$ is given by $\tau_{z} g(\omega)=$ $g\left(\tau_{z} \omega\right), \omega \in \Omega$. Hence $\mathcal{S}_{1}$ takes a function $A:[-\pi, \pi]^{d+1} \rightarrow \mathcal{M}$ to a function $\mathcal{S}_{1}(A)$ : $[\pi, \pi]^{d+1} \rightarrow L^{2}(\Omega, \mathcal{M})$. The operator $\mathcal{S}_{1}$ depends on the parameter $\operatorname{Re}(\eta)>0$. We shall obtain bounds on various norms of $\mathcal{S}_{1}$ which are independent of $\operatorname{Re}(\eta)$. The following two lemmas are analogous to Lemma 3.4 of [4]. 
Lemma 3.1. $\mathcal{S}_{1}$ extends to a bounded operator from $L^{\infty}\left([-\pi, \pi]^{d+1}, \mathcal{M}\right)$ to $L^{\infty}\left([-\pi, \pi]^{d+1}, L^{2}(\Omega, \mathcal{M})\right)$. The norm $\left\|\mathcal{S}_{1}\right\|_{\infty}$ of $\mathcal{S}_{1}$ satisfies the inequality

$$
\left\|\mathcal{S}_{1}\right\|_{\infty} \leq \sqrt{d}\left(\frac{\Lambda}{\lambda}\right)\left(1-\frac{\lambda}{\Lambda}\right)
$$

Proof. We put $\varphi=P \mathbf{b}\left[I-T_{\Lambda, \eta, \xi} P \mathbf{b}\right]^{-1}$. Now $T_{\Lambda, \eta, \xi}$ is bounded on $\mathcal{H}(\Omega)$ with norm not exceeding 1 . Hence for any column vector $\rho \in \mathbf{C}^{d}$ with adjoint $\rho^{*}$ there is the inequality

$$
\rho^{*}\left\langle\varphi(\cdot) \varphi^{*}(\cdot)\right\rangle \rho \leq\left(\frac{\Lambda}{\lambda}\right)^{2}\left(1-\frac{\lambda}{\Lambda}\right)^{2}|\rho|^{2} .
$$

It is clear that $\mathcal{S}_{1}(A)$ is given by the formula

$$
\mathcal{S}_{1}(A)(\zeta, \omega)=\sum_{y \in \mathbf{Z}^{d+1}} \hat{A}(y) e^{-i y \cdot \zeta} \varphi\left(\tau_{R y} \omega\right), \zeta \in[-\pi, \pi]^{d+1}, \omega \in \Omega .
$$

By Bochner's theorem there is a measure $d \mu_{\varphi}$ on $[-\pi, \pi]^{d+1}$, taking values in the self-adjoint nonnegative definite matrices on $\mathcal{M}$ with the property that

$$
\left\langle\varphi\left(\tau_{R y} \cdot\right) \varphi^{*}\left(\tau_{R y^{\prime}} \cdot\right)\right\rangle=\int_{[-\pi, \pi]^{d+1}} e^{i\left(y-y^{\prime}\right) \cdot \zeta^{\prime}} d \mu_{\varphi}\left(\zeta^{\prime}\right), \quad y, y^{\prime} \in \mathbf{Z}^{d+1} .
$$

Hence,

$$
\begin{gathered}
\left\langle\operatorname{Tr} \mathcal{S}_{1}(A)(\zeta, \cdot)^{*} \mathcal{S}_{1}(A)(\zeta, \cdot)\right\rangle \\
=\operatorname{Tr} \int_{[-\pi, \pi]^{d+1}} A\left(\zeta^{\prime}-\zeta\right) d \mu_{\varphi}\left(\zeta^{\prime}\right) A\left(\zeta^{\prime}-\zeta\right)^{*} \leq\|A\|_{\infty}^{2} \int_{[-\pi, \pi]^{d+1}} \operatorname{Tr} d \mu_{\varphi}\left(\zeta^{\prime}\right) .
\end{gathered}
$$

It follows now from (3.5), (3.6) that

$$
\operatorname{Tr} \int_{[-\pi, \pi]^{d+1}} A\left(\zeta^{\prime}-\zeta\right) d \mu_{\varphi}\left(\zeta^{\prime}\right) A\left(\zeta^{\prime}-\zeta\right)^{*} \leq d\left(\frac{\Lambda}{\lambda}\right)^{2}\left(1-\frac{\lambda}{\Lambda}\right)^{2}\|A\|_{\infty}^{2} .
$$

The inequality (3.4) follows from this last inequality.

Lemma 3.2. $\mathcal{S}_{1}$ extends to a bounded operator from $L^{2}\left([-\pi, \pi]^{d+1}, \mathcal{M}\right)$ to $L^{2}\left([-\pi, \pi]^{d+1}, L^{2}(\Omega, \mathcal{M})\right)$. The norm $\left\|\mathcal{S}_{1}\right\|_{2}$ of $\mathcal{S}_{1}$ satisfies the inequality

$$
\left\|\mathcal{S}_{1}\right\|_{2} \leq 2\left(\frac{\Lambda}{\lambda}\right)^{2}\left(1-\frac{\lambda}{\Lambda}\right)
$$

Proof. We write

$$
\begin{gathered}
\mathcal{S}_{1}=\sum_{m=0}^{\infty} \mathcal{S}_{1, m}, \text { where } \\
\mathcal{S}_{1, m}(A)(\zeta)=\sum_{y \in \mathbf{Z}^{d+1}} \hat{A}(y) e^{-i y \cdot \zeta} \tau_{R y} P \mathbf{b}\left(T_{\Lambda, \eta, \xi} P \mathbf{b}\right)^{m} .
\end{gathered}
$$

For $k=0,1,2, \ldots$ we define operators $\mathcal{S}_{1, k}^{\prime}$, similar to $\mathcal{S}_{1, k}$, by

$$
\mathcal{S}_{1, k}^{\prime}(A)(\zeta)=\sum_{y \in \mathbf{Z}^{d+1}} \hat{A}(y) e^{-i y \cdot \zeta} \tau_{R y} \mathbf{b}\left(T_{\Lambda, \eta, \xi} \mathbf{b}\right)^{k} .
$$


It is easy to see that there is the identity

$$
\begin{aligned}
& \mathcal{S}_{1, m}(A)(\zeta)=\mathcal{S}_{1, m}^{\prime}(A)(\zeta)-A(-\zeta)\left\langle\mathbf{b}\left(T_{\Lambda, \eta, \xi} P \mathbf{b}\right)^{m}\right\rangle \\
& -\sum_{k=0}^{m-1} \mathcal{S}_{1, k}^{\prime}(A)(\zeta) e(-\xi) e(-\xi)^{*} \Lambda e^{-\eta} \hat{G}_{\Lambda}(-\xi, \eta)\left\langle\mathbf{b}\left(T_{\Lambda, \eta, \xi} P \mathbf{b}\right)^{m-1-k}\right\rangle .
\end{aligned}
$$

Here $e(-\xi)^{*}$ is the row vector which is the adjoint of the column vector $e(-\xi)$. Hence the $d \times d$ matrix $e(-\xi) e(-\xi)^{*}$ is self-adjoint and

$$
\operatorname{Tr}\left[e(-\xi) e(-\xi)^{*}\right]^{2}=|e(-\xi)|^{4} .
$$

In view of (2.26) and the inequality

$$
\left|e^{\eta}-1+\gamma\right| \geq e^{\operatorname{Re}(\eta)} \gamma, \eta \in \mathbf{C}, \gamma \in \mathbf{R}, \operatorname{Re}(\eta) \geq 0, \quad 0 \leq \gamma \leq 1,
$$

we have that

$$
\Lambda|e(-\xi)|^{2}\left|\hat{G}_{\Lambda}(-\xi, \eta)\right| \leq 1,
$$

since we are assuming $4 d \Lambda \leq 1$. It follows from (3.10), (3.11), (3.13) and the boundedness of $T_{\Lambda, \eta, \xi}$ on $\mathcal{H}(\Omega)$ that

$$
\begin{aligned}
\left\|\mathcal{S}_{1, m}(A)\right\|_{2} & \leq\left\|\mathcal{S}_{1, m}^{\prime}(A)\right\|_{2}+\|A\|_{2}\left(1-\frac{\lambda}{\Lambda}\right)^{m+1} \\
& +\sum_{k=0}^{m-1}\left\|\mathcal{S}_{1, k}^{\prime}(A)\right\|_{2}\left(1-\frac{\lambda}{\Lambda}\right)^{m-k} .
\end{aligned}
$$

We need now to estimate $\left\|\mathcal{S}_{1, k}^{\prime}(A)\right\|_{2}$. To do this we write the operator $T_{\Lambda, \eta, \xi}$ of $(2.30)$ as

$$
T_{\Lambda, \eta, \xi} \varphi(\omega)=\sum_{y \in \mathbf{Z}^{d+1}} e^{-i y \cdot \zeta} \hat{F}(y) \varphi\left(\tau_{R y} \omega\right),
$$

where $\zeta=(\xi, \operatorname{Im}(\eta))$ and $F:[-\pi, \pi]^{d+1} \rightarrow \mathcal{M}$ is given by the formula

$$
F(\xi, \operatorname{Im}(\eta))=\Lambda e^{-\bar{\eta}} e(\xi) e(\xi)^{*} \hat{G}_{\Lambda}(\xi, \bar{\eta})
$$

where $\bar{\eta}$ is the complex conjugate of $\eta$. It follows from (2.26), (3.11), (3.13) that $F \in L^{\infty}\left([-\pi, \pi]^{d+1}, \mathcal{M}\right)$ and $\|F\|_{\infty} \leq 1$. From (3.9), 3.15) we have that

$$
\begin{gathered}
\mathcal{S}_{1, k}^{\prime}(A)(\zeta, \omega)=\sum_{y_{1}, \ldots, y_{k+1} \in \mathbf{Z}^{d+1}} \hat{A}\left(y_{1}\right) \exp \left[-i\left(y_{1}+\ldots+y_{k+1}\right) \cdot \zeta\right] \\
\mathbf{b}\left(\tau_{R y_{1}} \omega\right) \hat{F}\left(y_{2}\right) \mathbf{b}\left(\tau_{R\left(y_{1}+y_{2}\right)} \omega\right) \hat{F}\left(y_{3}\right) \cdots \hat{F}\left(y_{k+1}\right) \mathbf{b}\left(\tau_{R\left(y_{1}+\ldots+y_{k+1}\right)} \omega\right) .
\end{gathered}
$$

Hence,

$$
\begin{array}{r}
\int_{[-\pi, \pi]^{d+1}} \operatorname{Tr} \mathcal{S}_{1, k}^{\prime}(A)(\zeta, \omega)^{*} \mathcal{S}_{1, k}^{\prime}(A)(\zeta, \omega) d \zeta \\
=(2 \pi)^{d+1} \sum_{r \in \mathbf{Z}^{d+1}} \operatorname{Tr}\left[\Theta(r, \omega)^{*} \Theta(r, \omega)\right],
\end{array}
$$

where

$$
\begin{aligned}
\Theta(r, \omega)=\sum_{y_{1}+. .+y_{k+1}=r} \hat{A}\left(y_{1}\right) \mathbf{b}\left(\tau_{R y_{1}} \omega\right) \hat{F}\left(y_{2}\right) & \\
& \mathbf{b}\left(\tau_{R\left(y_{1}+y_{2}\right)} \omega\right) \hat{F}\left(y_{3}\right) \cdots \hat{F}\left(y_{k+1}\right) \mathbf{b}\left(\tau_{R\left(y_{1}+. . y_{k+1}\right)} \omega\right) .
\end{aligned}
$$


Note that, in view of (2.21), (3.1), there exists $B_{\omega} \in L^{2}\left([-\pi, \pi]^{d+1}, \mathcal{M}\right)$ such that

$$
\hat{B}_{\omega}(y)=\hat{A}(y) \mathbf{b}\left(\tau_{R y} \omega\right), \quad y \in \mathbf{Z}^{d+1}, \quad\left\|B_{\omega}\right\|_{2} \leq\left(1-\frac{\lambda}{\Lambda}\right)\|A\|_{2} .
$$

We may also define an operator $T_{\omega}$ on $L^{2}\left([-\pi, \pi]^{d+1}, \mathcal{M}\right)$ by

$$
\mathcal{F}\left(T_{\omega} G\right)(y)=\sum_{y^{\prime} \in \mathbf{Z}^{d+1}} \hat{G}\left(y^{\prime}\right) \hat{F}\left(y-y^{\prime}\right) \mathbf{b}\left(\tau_{R y} \omega\right), \quad y \in \mathbf{Z}^{d+1} .
$$

Since $\|F\|_{\infty} \leq 1$, it follows that $T_{\omega}$ is a bounded operator on $L^{2}\left([-\pi, \pi]^{d+1}, \mathcal{M}\right)$ and $\left\|T_{\omega}\right\|_{2} \leq(1-\lambda / \Lambda)$. We also see that

$$
\Theta(r, \omega)=\mathcal{F}\left(T_{\omega}^{k} B_{\omega}\right)(r), \quad r \in \mathbf{Z}^{d+1} .
$$

Hence from (3.1), (3.17) we have that

$$
\begin{gathered}
\int_{[-\pi, \pi]^{d+1}} \operatorname{Tr} \mathcal{S}_{1, k}^{\prime}(A)(\zeta, \omega)^{*} \mathcal{S}_{1, k}^{\prime}(A)(\zeta, \omega) d \zeta \\
=\left\|T_{\omega}^{k} B_{\omega}\right\|_{2}^{2} \leq\left(1-\frac{\lambda}{\Lambda}\right)^{2(k+1)}\|A\|_{2}^{2} .
\end{gathered}
$$

Taking the expectation of the last inequality, we conclude that

$$
\left\|\mathcal{S}_{1, k}^{\prime}\right\| \leq\left(1-\frac{\lambda}{\Lambda}\right)^{k+1}
$$

It follows now from (3.14) that

$$
\left\|\mathcal{S}_{1, m}\right\| \leq(m+2)\left(1-\frac{\lambda}{\Lambda}\right)^{m+1}
$$

The inequality (3.7) follows from this last inequality and (3.8).

Corollary 3.1. For $2 \leq p \leq \infty$ the operator $\mathcal{S}_{1}$ extends to a bounded operator from $L^{p}\left([-\pi, \pi]^{d+1}, \mathcal{M}\right)$ to $L^{p}\left([-\pi, \pi]^{d+1}, L^{2}(\Omega, \mathcal{M})\right)$. The norm $\left\|\mathcal{S}_{1}\right\|_{p}$ of $\mathcal{S}_{1}$ satisfies the inequality

$$
\left\|\mathcal{S}_{1}\right\|_{p} \leq 2^{2 / p} d^{1 / 2-1 / p}\left(\frac{\Lambda}{\lambda}\right)^{1+2 / p}\left(1-\frac{\lambda}{\Lambda}\right) .
$$

Proof. This is a consequence of Lemmas 3.1, 3.2 and the Riesz convexity theorem [10] for Banach space-valued functions (p. 212 of [10]).

Corollary 3.2. For $2<p<\infty$ the operator $\mathcal{S}_{1}$ extends to a bounded operator from $L_{w}^{p}\left([-\pi, \pi]^{d+1}, \mathcal{M}\right)$ to $L_{w}^{p}\left([-\pi, \pi]^{d+1}, L^{2}(\Omega, \mathcal{M})\right)$. The norm $\left\|\mathcal{S}_{1}\right\|_{p, w}$ of $\mathcal{S}_{1}$ satisfies the inequality

$$
\left\|\mathcal{S}_{1}\right\|_{p, w} \leq C_{p} d^{1 / 2-1 / p}\left(\frac{\Lambda}{\lambda}\right)^{1+2 / p}\left(1-\frac{\lambda}{\Lambda}\right)
$$

where $C_{p}$ is a constant depending only on $p$.

Proof. This is a consequence of Lemmas 3.1, 3.2 and the Hunt interpolation theorem [9]. 
We consider next multilinear operators on functions $A:[-\pi, \pi]^{d+1} \rightarrow \mathcal{M}$ with the property that $\hat{A}$ has finite support in $\mathbf{Z}^{d+1}$. Suppose we have $N$ such functions $A_{1}, \ldots, A_{N}$. Then we define the mapping $\mathcal{S}_{N}\left(A_{1}, \ldots, A_{N}\right)$ from $[-\pi, \pi]^{d+1}$ to $L^{2}(\Omega, \mathcal{M})$ by

$$
\begin{aligned}
& \mathcal{S}_{N}\left(A_{1}, \ldots, A_{N}\right)(\zeta) \\
& \quad=\sum_{y_{1}, . ., y_{N} \in \mathbf{Z}^{d+1}} \prod_{k=1}^{N}\left\{\hat{A}_{k}\left(y_{k}\right) e^{-i y_{k} \cdot \zeta} \tau_{R y_{k}} P \mathbf{b}\left[I-T_{\Lambda, \eta, \xi} P \mathbf{b}\right]^{-1}\right\} .
\end{aligned}
$$

The following is the analog of Lemma 3.7 of [4].

Lemma 3.3. Suppose $\infty \geq p_{1}, \ldots, p_{N}, p \geq 2$ and $1 / p_{1}+\ldots+1 / p_{N}=1 / p$. Then $\mathcal{S}_{N}$ extends to a bounded multilinear operator from

$$
\prod_{k=1}^{N} L^{p_{k}}\left([-\pi, \pi]^{d+1}, \mathcal{M}\right) \quad \text { to } \quad L^{p}\left([-\pi, \pi]^{d+1}, L^{2}(\Omega, \mathcal{M})\right) .
$$

Further, one has the norm bound

$$
\left\|\mathcal{S}_{N}\left(A_{1}, \ldots, A_{N}\right)\right\|_{p} \leq(\sqrt{d}+1)^{N}\left(\frac{\Lambda}{\lambda}\right)^{2 N}\left(1-\frac{\lambda}{\Lambda}\right)^{N} \prod_{k=1}^{N}\left\|A_{k}\right\|_{p_{k}} .
$$

Proof. Note that Corollary 3.1 implies the result for $N=1$. We shall use induction to prove it for $N>1$. Observe that by the argument of Lemma 3.1 we have the inequality

$$
\left\|\mathcal{S}_{N}\left(A_{1}, \ldots, A_{N}\right)\right\|_{p} \leq \sqrt{d}\left(\frac{\Lambda}{\lambda}\right)\left(1-\frac{\lambda}{\Lambda}\right)\left\|A_{1}\right\|_{p_{1}}\left\|\mathcal{S}_{N-1}\left(A_{2}, \ldots, A_{N}\right)\right\|_{p},
$$

provided $p_{1}=\infty$. We also have by the argument of Lemma 3.2 that

$$
\left\|\mathcal{S}_{N}\left(A_{1}, \ldots, A_{N}\right)\right\|_{2} \leq 2\left(\frac{\Lambda}{\lambda}\right)^{2}\left(1-\frac{\lambda}{\Lambda}\right)\left\|\mathcal{S}_{N-1}\left(A_{1}, \ldots, A_{N-1}\right) \tilde{A}_{N}\right\|_{2},
$$

where $\tilde{A}_{N}$ is given by $\tilde{A}_{N}(\zeta)=A_{N}(-\zeta), \zeta \in[-\pi, \pi]^{d+1}$. Hence if we choose $q \geq 2$ to satisfy $1 / q+1 / p_{N}=1 / 2$, we have, by Holder's inequality,

$$
\left\|\mathcal{S}_{N}\left(A_{1}, \ldots, A_{N}\right)\right\|_{2} \leq 2\left(\frac{\Lambda}{\lambda}\right)^{2}\left(1-\frac{\lambda}{\Lambda}\right)\left\|\mathcal{S}_{N-1}\left(A_{1}, \ldots, A_{N-1}\right)\right\|_{q}\left\|A_{N}\right\|_{p_{N}} .
$$

We have already seen that the inequality (3.19) holds for $N=1$. Suppose now it holds up to $N=m-1$ for some $m \geq 2$. We show it holds for $N=m$. To see this consider $A_{2}, \ldots, A_{m}$ as fixed functions with $A_{k} \in L^{p_{k}}\left([-\pi, \pi]^{d+1}, \mathcal{M}\right), 2 \leq k \leq m$, where $1 / p_{2}+\cdots+1 / p_{k} \leq 1 / 2$. We consider the linear operator $\mathcal{S}$ on functions $A_{1}:[-\pi, \pi]^{d+1} \rightarrow \mathcal{M}$, defined by $\mathcal{S}\left(A_{1}\right)=\mathcal{S}_{m}\left(A_{1}, . ., A_{m}\right)$. If $p_{1}=\infty$ and hence satisfies the identity

$$
\frac{1}{p}=\frac{1}{p_{1}}+\cdots+\frac{1}{p_{m}}=\frac{1}{p_{2}}+\cdots+\frac{1}{p_{m}},
$$

then (3.20) and the induction assumption implies that $\mathcal{S}$ is a bounded operator from

$$
L^{p_{1}}\left([-\pi, \pi]^{d+1}, \mathcal{M}\right) \text { to } L^{p}\left([-\pi, \pi]^{d+1}, L^{2}(\Omega, \mathcal{M})\right)
$$


and

$$
\|\mathcal{S}\| \leq 2^{m}\left(\frac{\Lambda}{\lambda}\right)^{2 m}\left(1-\frac{\lambda}{\Lambda}\right)^{m} \prod_{k=2}^{m}\left\|A_{k}\right\|_{p_{k}} .
$$

If $p_{1} \geq 2$ satisfies the identity

$$
\frac{1}{p}=\frac{1}{p_{1}}+\cdots+\frac{1}{p_{m}}=\frac{1}{2}
$$

then (3.21) and the induction assumption implies that $\mathcal{S}$ is a bounded operator from $L^{p_{1}}\left([-\pi, \pi]^{d+1}, \mathcal{M}\right)$ to $L^{p}\left([-\pi, \pi]^{d+1}, L^{2}(\Omega, \mathcal{M})\right)$ and the inequality (3.22) holds. Now we can apply the Riesz convexity theorem as in Corollary 3.1 to conclude that if $p_{1} \geq 2$ satisfies

$$
\frac{1}{p_{1}}+\cdots+\frac{1}{p_{m}}=\frac{1}{p} \leq \frac{1}{2}
$$

then $\mathcal{S}$ is again a bounded operator from

$$
L^{p_{1}}\left([-\pi, \pi]^{d+1}, \mathcal{M}\right) \quad \text { to } \quad L^{p}\left([-\pi, \pi]^{d+1}, L^{2}(\Omega, \mathcal{M})\right)
$$

and (3.22) holds. Hence (3.19) holds for $N=m$.

Lemma 3.4. Suppose $\infty>p_{1}, \ldots, p_{N}, p>2$ and $1 / p_{1}+\cdots+1 / p_{N}=1 / p$. Then $\mathcal{S}_{N}$ extends to a bounded multilinear operator from

$$
\prod_{k=1}^{N} L_{w}^{p_{k}}\left([-\pi, \pi]^{d+1}, \mathcal{M}\right) \quad \text { to } \quad L_{w}^{p}\left([-\pi, \pi]^{d+1}, L^{2}(\Omega, \mathcal{M})\right) .
$$

Further, there is a constant $C\left(p_{1}, \ldots, p_{N}\right)$, depending only on $p_{1}, \ldots, p_{N}$, such that

$$
\left\|\mathcal{S}_{N}\left(A_{1}, \ldots, A_{N}\right)\right\|_{p, w} \leq C\left(p_{1}, \ldots, p_{N}\right)\left(\frac{\Lambda}{\lambda}\right)^{2 N}\left(1-\frac{\lambda}{\Lambda}\right)^{N} \prod_{k=1}^{N}\|A\|_{p_{k}, w} .
$$

Proof. Let $K \subset \mathbf{R}^{N}$ be the convex set

$$
K=\left\{\left(x_{1}, \ldots, x_{N}\right): x_{i} \geq 0, i=1, \ldots, N, x_{1}+\cdots+x_{N} \leq 1 / 2\right\} .
$$

Lemma 3.3 shows that the inequality (3.19) holds provided $\left(1 / p_{1}, \ldots, 1 / p_{N}\right) \in K$. Here we wish to prove that (3.23) holds provided $\left(1 / p_{1}, \ldots, 1 / p_{N}\right)$ is in the interior of $K$. It follows from the Hunt interpolation theorem and Lemma 3.3 that

$$
\left\|\mathcal{S}_{N}\left(A_{1}, \ldots, A_{N}\right)\right\|_{p, w} \leq C\left(p_{1}, \ldots, p_{N}\right)\left(\frac{\Lambda}{\lambda}\right)^{2 N}\left(1-\frac{\lambda}{\Lambda}\right)^{N}\left\|A_{1}\right\|_{p_{1}, w} \prod_{k=2}^{N}\left\|A_{k}\right\|_{p_{k}},
$$

provided $\left(1 / p_{1}, \ldots, 1 / p_{N}\right)$ is in the interior of $K$. Next we suppose $A_{1} \in L_{w}^{p_{1}}, A_{k} \in$ $L^{p_{k}}, \quad k \geq 3$ are fixed and consider the linear mapping

$$
A_{2} \rightarrow \mathcal{S}_{N}\left(A_{1}, A_{2}, \ldots, A_{N}\right) .
$$

By (3.24) this maps $L^{p_{2}}$ to $L_{w}^{p}$ provided $\left(1 / p_{1}, \ldots, 1 / p_{N}\right)$ lies in the interior of $K$. By the general interpolation theorem, Theorem 3.15 from Chapter V of [10], it follows 
that it also maps $L_{w}^{p_{2}}$ to $L_{w}^{p}$ provided $\left(1 / p_{1}, \ldots, 1 / p_{N}\right)$ lies in the interior of $K$, and there is the inequality

$$
\begin{aligned}
& \left\|\mathcal{S}_{N}\left(A_{1}, \ldots, A_{N}\right)\right\|_{p, w} \\
& \quad \leq C^{\prime}\left(p_{1}, \ldots, p_{N}\right)\left(\frac{\Lambda}{\lambda}\right)^{2 N}\left(1-\frac{\lambda}{\Lambda}\right)^{N}\left\|A_{1}\right\|_{p_{1}, w}\left\|A_{2}\right\|_{p_{2}, w} \prod_{k=3}^{N}\left\|A_{k}\right\|_{p_{k}},
\end{aligned}
$$

for a constant $C^{\prime}\left(p_{1}, \ldots, p_{N}\right)$ depending only on $p_{1}, \ldots, p_{N}$. It is clear that now we can continue to apply this argument to obtain (3.23).

We have already seen in Lemma 2.4 that the function $q(\xi, \eta)$ defined by (2.13) is a $C^{\infty}$ function of $(\xi, \eta)$ for $\xi \in \mathbf{R}^{d}, \eta \in \mathbf{C}, \operatorname{Re}(\eta)>0$, and analytic in $\eta$. Lemmas 2.7 and 2.8 give us estimates on $q(\xi, \eta)$ which are independent of $\operatorname{Re}(\eta)$ as $\operatorname{Re}(\eta) \rightarrow 0$. We shall use Lemma 3.4 now to obtain similar estimates on derivatives of $q(\xi, \eta)$. In the following we shall denote the integer part of a nonnegative number $x$ by $[x]$.

Lemma 3.5. Suppose $4 d \Lambda \leq 1, \operatorname{Re}(\eta)>0, \xi \in[-\pi, \pi]^{d}$. For fixed $\operatorname{Re}(\eta)>0$ consider $q(\xi, \eta)$ as a function of $\zeta=(\xi, \operatorname{Im}(\eta)) \in[-\pi, \pi]^{d+1}$. Then for $1 \leq m \leq$ $\left[\frac{d-1}{2}\right]+1$, the derivatives $\partial^{m} q / \partial \eta^{m}$ are in $L_{w}^{(1+d / 2) / m}\left([-\pi, \pi]^{d+1}, \mathcal{M}\right)$. Further, there is a constant $C_{d}$ depending only on $d$ such that

$$
\left\|\frac{\partial^{m} q}{\partial \eta^{m}}\right\|_{(1+d / 2) / m, w} \leq C_{d} \Lambda^{1-m d /(d+2)}\left(\frac{\Lambda}{\lambda}\right)^{2(m+1)} .
$$

Proof. From (2.24), (2.31) we have that

$$
\begin{aligned}
q(\xi, \eta) & =\langle\mathbf{a}(\cdot)\rangle-\Lambda\langle\mathbf{b}(\cdot) \Psi(\xi, \eta, \cdot)\rangle \\
& =\langle\mathbf{a}(\cdot)\rangle-\Lambda\left\langle\mathbf{b}(\cdot) \sum_{n=1}^{\infty}\left[T_{\Lambda, \eta, \xi} P \mathbf{b}(\cdot)\right]^{n}\right\rangle .
\end{aligned}
$$

Hence from (2.33) we have that

$$
\frac{\partial q}{\partial \eta}(\xi, \eta)=-\Lambda\left\langle\mathbf{b}(\cdot)\left[I-T_{\Lambda, \eta, \xi} P \mathbf{b}\right]^{-1}\left[\frac{\partial}{\partial \eta} T_{\Lambda, \eta, \xi} P \mathbf{b}\right]\left[I-T_{\Lambda, \eta, \xi} P \mathbf{b}\right]^{-1}\right\rangle
$$

Observe now that

$$
\left[\frac{\partial}{\partial \eta} T_{\Lambda, \eta, \xi} P \mathbf{b}\right]\left[I-T_{\Lambda, \eta, \xi} P \mathbf{b}\right]^{-1}=\mathcal{S}_{1}(A)(\zeta)
$$

where $A(\zeta)=A(\xi, \operatorname{Im}(\eta))$ is given by

$$
A(\zeta)=i \frac{\partial F(\xi, \operatorname{Im}(\eta))}{\partial[\operatorname{Im}(\eta)]}
$$

and $F$ is the function defined by (3.16). From $(2.26)$ we see then that

$$
A(\zeta)=-\frac{e^{\bar{\eta}}}{\left[e^{\bar{\eta}}-1+\Lambda|e(\xi)|^{2}\right]} F(\xi, \operatorname{Im}(\eta)) .
$$


We have already observed that $F \in L^{\infty}\left([-\pi, \pi]^{d+1}, \mathcal{M}\right)$ with $\|F\|_{\infty} \leq 1$. Note also the inequalities

$$
\begin{gathered}
\left|e^{\eta}-1+\gamma\right| \geq \frac{2}{\pi} e^{\operatorname{Re}(\eta)}|\operatorname{Im}(\eta)|, \eta \in \mathbf{C},|\operatorname{Im}(\eta)| \leq \frac{\pi}{2}, \gamma \in \mathbf{R}, \\
\left|e^{\eta}-1+\gamma\right| \geq e^{\operatorname{Re}(\eta)}, \eta \in \mathbf{C}, \operatorname{Re}(\eta) \geq 0, \\
\frac{\pi}{2} \leq|\operatorname{Im}(\eta)| \leq \pi, \gamma \in \mathbf{R}, 0 \leq \gamma \leq 1 .
\end{gathered}
$$

It follows from (3.12), (3.30), (3.31) that

$$
\begin{aligned}
\left|\frac{e^{\bar{\eta}}}{\left.\left.\left|e^{\bar{\eta}}-1+\Lambda\right| e(\xi)\right|^{2}\right]}\right| \leq 1, \frac{\pi}{2} \leq|\operatorname{Im}(\eta)| \leq \pi, \\
\left|\frac{e^{\bar{\eta}}}{\left[e^{\bar{\eta}}-1+\Lambda|e(\xi)|^{2}\right]}\right| \leq \frac{2 \pi}{2|\operatorname{Im}(\eta)|+\pi \Lambda|e(\xi)|^{2}},|\operatorname{Im}(\eta)| \leq \frac{\pi}{2} .
\end{aligned}
$$

We conclude that the function $A(\zeta)$ of $(3.29)$ is in $L_{w}^{1+d / 2}\left([-\pi, \pi]^{d+1}, \mathcal{M}\right)$ and there is a constant $C_{d}$ depending only on $d$ such that

$$
\|A\|_{1+d / 2, w} \leq C_{d} \Lambda^{-d /(d+2)} .
$$

Now Corollary 3.2 implies that $\mathcal{S}_{1}(A)$ is in $L_{w}^{1+d / 2}\left([-\pi, \pi]^{d+1}, L^{2}(\Omega, \mathcal{M})\right)$ provided $d \geq 3$, and

$$
\left\|\mathcal{S}_{1}(A)\right\|_{1+d / 2, w} \leq C_{d} \Lambda^{-d /(d+2)}\left(\frac{\Lambda}{\lambda}\right)^{2},
$$

for some constant $C_{d}$ depending only on $d$. From (3.27), (3.28) we have that

$$
\left\|\frac{\partial q}{\partial \eta}(\xi, \eta)\right\| \leq \Lambda\left(1-\frac{\lambda}{\Lambda}\right)\left(\frac{\Lambda}{\lambda}\right)\left\|\mathcal{S}_{1}(A)(\zeta)\right\|,
$$

where $\zeta=(\xi, \operatorname{Im}(\eta))$. The inequality (3.25) follows now from the previous two inequalities provided $d \geq 3, m=1$.

We consider the cases $d=1,2, \ldots, m=1$. Note that the convolution formula (3.2) implies that

$$
\begin{aligned}
& \sum_{y \in \mathbf{Z}^{d+1}} \mathcal{F}(A B)(y) e^{-i y \cdot \zeta} \tau_{R y} \\
& \quad=\sum_{y \in \mathbf{Z}^{d+1}} \hat{A}(y) e^{-i y \cdot \zeta} \tau_{R y} \sum_{z \in \mathbf{Z}^{d+1}} \hat{B}(z) e^{-i z \cdot \zeta} \tau_{R z} .
\end{aligned}
$$

Hence, if we write the matrix $A(\zeta)$ of (3.29) as $A(\zeta)=A_{1}(\zeta)^{*} A_{2}(\zeta)$, then we see from (3.27) and (3.33) that

$$
\frac{\partial q}{\partial \eta}(\xi, \eta)=-\Lambda\left\langle\mathcal{S}_{1}^{\prime}\left(A_{1}\right)(\zeta, \cdot)^{*} \mathcal{S}_{1}\left(A_{2}\right)(\zeta, \cdot)\right\rangle,
$$

where the operator $\mathcal{S}_{1}^{\prime}$ is defined in exactly the same manner as the operator $\mathcal{S}_{1}$ by (3.3). The only difference is that the operator $T_{\Lambda, \eta, \xi}$ on $L^{2}(\Omega, \mathcal{M})$ is replaced by the operator $T_{\Lambda, \eta, \xi}^{*}$ on $L^{2}(\Omega, \mathcal{M})$. The operator $T_{\Lambda, \eta, \xi}^{*}$ is defined just like $T_{\Lambda, \eta, \xi}$ as in (3.15). The difference is that the function $F(\zeta)$ of (3.16) is replaced by $F(\zeta)^{*}$. Evidently $T_{\Lambda, \eta, \xi}^{*}$ is the adjoint of $T_{\Lambda, \eta, \xi}$ as an operator on $L^{2}(\Omega, \mathcal{M})$. Now we choose $A_{1}(\zeta)$ to be

$$
A_{1}(\zeta)=\left[1-e^{\eta}\left\{1-\Lambda|e(\xi)|^{2}\right\}\right]^{-1 / 2} I_{d}
$$


where $I_{d}$ is the identity $d \times d$ matrix. Note that $A_{1}(\zeta)$ is analytic in $\eta$ for $\operatorname{Re}(\eta)>0$. From (3.29) we see that $A_{2}(\zeta)$ is given by

$$
A_{2}(\zeta)=-\left[1-e^{\bar{\eta}}\left\{1-\Lambda|e(\xi)|^{2}\right\}\right]^{-1 / 2} F(\xi, \operatorname{Im}(\eta)) .
$$

We see from (3.32) that $A_{1}$ and $A_{2}$ are in $L_{w}^{2+d}\left([-\pi, \pi]^{d+1}, \mathcal{M}\right)$ and there is a constant $C_{d}$ depending only on $d$ such that

$$
\left\|A_{1}\right\|_{2+d, w}+\left\|A_{2}\right\|_{2+d, w} \leq C_{d} \Lambda^{-d / 2(d+2)} .
$$

Hence by Corollary $3.2 \mathcal{S}_{1}^{\prime}\left(A_{1}\right)$ and $\mathcal{S}_{1}\left(A_{2}\right)$ are in $L_{w}^{2+d}\left([-\pi, \pi]^{d+1}, L^{2}(\Omega, \mathcal{M})\right)$ for $d \geq 1$. Further, we have

$$
\left\|\mathcal{S}_{1}^{\prime}\left(A_{1}\right)\right\|_{2+d, w}+\left\|\mathcal{S}_{1}\left(A_{2}\right)\right\|_{2+d, w} \leq C_{d} \Lambda^{-d / 2(d+2)}\left(\frac{\Lambda}{\lambda}\right)^{2} .
$$

The inequality (3.25) follows now from the previous inequality and (3.34). Here we have used the fact that if $A, B \in L^{2}(\Omega, \mathcal{M})$, then $\langle A(\cdot) B(\cdot)\rangle \in \mathcal{M}$ and

$$
\|\langle A(\cdot) B(\cdot)\rangle\| \leq\|A\|\|B\| .
$$

We have proved the result for $m=1$.

To deal with the case $m \geq 1$ we differentiate (3.27). Thus $\partial^{m} q / \partial \eta^{m}(\xi, \eta)$ is a sum of terms of the form

$$
-\Lambda\left\langle\mathbf{b}(\cdot)\left[I-T_{\Lambda, \eta, \xi} P \mathbf{b}\right]^{-1} \mathcal{S}_{k}\left(A_{1}, \ldots, A_{k}\right)(\zeta)\right\rangle,
$$

where $k \leq m, A_{j} \in L_{w}^{p_{j}}\left([-\pi, \pi]^{d+1}, \mathcal{M}\right), p_{j}=(d+2) / 2 r_{j}, \quad 1 \leq j \leq k$, and the $r_{j}$ are nonnegative integers satisfying $r_{1}+\cdots+r_{k}=m$. If $m \leq[(d-1) / 2]+1$, there is a constant $C_{d}$ depending only on $d$ such that

$$
\left\|A_{j}\right\|_{p_{j}, w} \leq C_{d} \Lambda^{-d r_{j} /(d+2)}, 1 \leq j \leq k .
$$

If $(d+2) / 2 m>2$, then by Lemma 3.4 and the previous inequality we have that the function (3.36) is in $L_{w}^{(1+d / 2) / m}\left([-\pi, \pi]^{d+1}, \mathcal{M}\right)$ and its norm is bounded by

$$
\Lambda\left(\frac{\Lambda}{\lambda}\right) C\left(p_{1}, \ldots, p_{k}\right)\left(\frac{\Lambda}{\lambda}\right)^{2 k} \prod_{j=1}^{k}\left\|A_{j}\right\|_{p_{j}, w} \leq C_{d} \Lambda^{1-m d /(d+2)}\left(\frac{\Lambda}{\lambda}\right)^{2 m+1}
$$

where $C_{d}$ is a constant depending only on $d$. Hence (3.25) follows provided $(d+2) / 2 m>2$. To finish the proof note that if $m \leq[(d-1) / 2]+1$, then $(d+2) / 2 m>1$. Suppose now $1<(d+2) / 2 m \leq 2$. Let $k^{\prime}$ be the smallest integer such that $4\left(r_{1}+\ldots+r_{k^{\prime}}\right) \geq d+2$. Then we may write $r_{k^{\prime}}=r_{k^{\prime}, 1}+r_{k^{\prime}, 2}$ with

$$
m_{1}=r_{1}+r_{2}+\ldots+r_{k^{\prime}-1}+r_{k^{\prime}, 1}=\frac{d+2}{4}-\frac{1}{8} .
$$

Evidently $(1+d / 2) / m_{1}>2$. Putting $m_{2}=m-m_{1}$, it is also clear that $(1+d / 2) / m_{2}>2$. We can write the matrix $A_{k^{\prime}}(\zeta)$ as $A_{k^{\prime}}(\zeta)=A_{k^{\prime}, 1}(\zeta)^{*} A_{k^{\prime}, 2}(\zeta)$ where

$$
A_{k^{\prime}, j} \in L_{w}^{p_{k^{\prime}, j}}\left([-\pi, \pi]^{d+1}, \mathcal{M}\right), j=1,2,
$$

with $p_{k^{\prime}, j}=(d+2) / 2 r_{k^{\prime}, j}$,

$$
\left\|A_{k^{\prime}, j}\right\|_{p_{k^{\prime}, j}, w} \leq C_{d} \Lambda^{-r_{k^{\prime}, j} d /(d+2)}, j=1,2,
$$


and $C_{d}$ depends only on $d$. We also have that the expression (3.36) is identical to

$$
\begin{aligned}
-\Lambda\left\langle\mathcal { S } _ { k ^ { \prime } } ^ { \prime } \left( A_{k^{\prime}, 1}, A_{k^{\prime}-1}^{*}, A_{k^{\prime}-2}^{*}, \ldots,\right.\right. & \left.A_{1}^{*}\right)(\zeta, \cdot)^{*} \\
& \left.\mathcal{S}_{k+1-k^{\prime}}\left(A_{k^{\prime}, 2}, A_{k^{\prime}+1}, \ldots, A_{k}\right)(\zeta, \cdot)\right\rangle .
\end{aligned}
$$

Here the operator $\mathcal{S}_{N}^{\prime}$ is defined in the same way as the operator $\mathcal{S}_{N}$ of (3.18), the only difference being that the operator $T_{\Lambda, \eta, \xi}$ is replaced by its adjoint $T_{\Lambda, \eta, \xi}^{*}$ on $L^{2}(\Omega, \mathcal{M})$. The functions $A_{j}^{*}$ are defined by $A_{j}^{*}(\zeta)=A_{j}(\zeta)^{*}, \zeta \in[-\pi, \pi]^{d+1}$. Evidently Lemma 3.4 also applies to the operators $\mathcal{S}_{N}^{\prime}$. Hence the inequality (3.25) follows from (3.35), (3.37) and Lemma 3.4.

Lemma 3.6. Suppose $4 d \Lambda \leq 1, \operatorname{Re}(\eta)>0, \xi \in[-\pi, \pi]^{d}$. For fixed $\operatorname{Re}(\eta)>0$ consider $q(\xi, \eta)$ as a function of $\zeta=(\xi, \operatorname{Im}(\eta)) \in[-\pi, \pi]^{d+1}$. Let $m=\left[\frac{d-1}{2}\right]+1$, and $\varepsilon \in \mathbf{R}$ satisfy $|\varepsilon|<1$. Then for any $\alpha \geq 0$ satisfying $m+\alpha<1+d / 2$ the function

$$
\frac{1}{|\varepsilon|^{\alpha}}\left\{\frac{\partial^{m} q}{\partial \eta^{m}}(\xi, \operatorname{Im}(\eta)+\varepsilon)-\frac{\partial^{m} q}{\partial \eta^{m}}(\xi, \operatorname{Im}(\eta))\right\}
$$

is in $L_{w}^{(1+d / 2) /(m+\alpha)}\left([-\pi, \pi]^{d+1}, \mathcal{M}\right)$. Further, there is a constant $C_{d, \alpha}$ depending only on $d$ and $\alpha$ such that the function (3.38) has norm bounded as

$$
\|\cdot\|_{(1+d / 2) /(m+\alpha), w} \leq C_{d, \alpha} \Lambda^{1-(m+\alpha) d /(d+2)}\left(\frac{\Lambda}{\lambda}\right)^{d+5} .
$$

Proof. We see from (3.26) that

$$
\begin{aligned}
& \frac{1}{|\varepsilon|^{\alpha}}\{q(\xi, \eta+i \varepsilon)-q(\xi, \eta)\}= \\
& -\Lambda\left\langle\mathbf{b}(\cdot)\left[I-T_{\Lambda, \eta+i \varepsilon, \xi} P \mathbf{b}\right]^{-1} \frac{\left[T_{\Lambda, \eta+i \varepsilon, \xi}-T_{\Lambda, \eta, \xi}\right]}{|\varepsilon|^{\alpha}} P \mathbf{b}\left[I-T_{\Lambda, \eta, \xi} P \mathbf{b}\right]^{-1}\right\rangle .
\end{aligned}
$$

We see similarly to 3.28 that

$$
\frac{1}{|\varepsilon|^{\alpha}}\left[T_{\Lambda, \eta+i \varepsilon, \xi}-T_{\Lambda, \eta, \xi}\right] P \mathbf{b}\left[I-T_{\Lambda, \eta, \xi} P \mathbf{b}\right]^{-1}=\mathcal{S}_{1}(A)(\zeta)
$$

where $A(\zeta)=A(\xi, \operatorname{Im}(\eta))$ is given by

$$
A(\zeta)=[F(\xi, \operatorname{Im}(\eta)-\varepsilon)-F(\xi, \operatorname{Im}(\eta))] /|\varepsilon|^{\alpha},
$$

and $F$ is defined by (3.16). Observe now that for any $\alpha, 0 \leq \alpha \leq 1$, the function

$$
\frac{1}{|\varepsilon|^{\alpha}}\left\{\frac{e^{\eta-i \varepsilon}}{\left[e^{\eta-i \varepsilon}-1+\Lambda|e(\xi)|^{2}\right]}-\frac{e^{\eta}}{\left[e^{\eta}-1+\Lambda|e(\xi)|^{2}\right]}\right\}
$$

is in $L_{w}^{(1+d / 2) /(\alpha+1)}\left([-\pi, \pi]^{d+1}, \mathbf{C}\right)$ with the norm of the function (3.41) bounded as

$$
\|\cdot\|_{(1+d / 2) /(\alpha+1), w} \leq C_{d} \Lambda^{-d(\alpha+1) /(d+2)},
$$

where $C_{d}$ is a constant depending only on $d$. The result follows now by differentiating (3.40) $m$ times and using the argument of Lemma 3.5. 
Remark 5. The conditions on $\alpha$ in Lemma 3.6 are $\alpha<1 / 2$ if $d$ is odd and $\alpha<1$ if $d$ is even. As $\alpha$ approaches its upper bound the constant $C_{d, \alpha}$ of (3.39) diverges. This is a consequence of the fact that as $\alpha$ approaches its upper bound the corresponding value of $p$ in Lemma 3.4 approaches 2 .

\section{Proof of Theorem 1.1}

Since the function $\hat{G}_{\mathbf{a}}(\xi, \eta)$ of (2.1) $), \xi \in[-\pi, \pi]^{d}, \operatorname{Re}(\eta)>0$, is $C^{\infty}$ and analytic in $\eta$, it follows from (2.2) that for any integer $m \geq 1$, there is the identity (4.1)

$$
(2 \pi)^{d+1} G_{\mathbf{a}}(x, t)=\frac{(-1)^{m}}{t^{m}} \int_{[-\pi, \pi]^{d}} d \xi e^{-i x \cdot \xi} \int_{-\pi}^{\pi} d[\operatorname{Im}(\eta)]\left\{e^{\eta t}-1\right\} \frac{\partial^{m} \hat{G}_{\mathbf{a}}(\xi, \eta)}{\partial \eta^{m}} .
$$

Lemma 4.1. Suppose $d$ is odd, $4 d \Lambda \leq 1$ and $x \in \mathbf{Z}^{d}, t \in \mathbf{Z}, t \geq 0$. Then there is a positive constant $C_{d}$, depending only on $d$, such that

$$
\left|G_{\mathbf{a}}(x, t)\right| \leq \frac{C_{d}(\Lambda / \lambda)^{3 d+4}}{1+(\Lambda t)^{d / 2}} .
$$

Proof. The inequality (4.2) follows from Lemmas 2.9 and 2.10 if $\Lambda t \leq 1$, whence we may assume $\Lambda t>1$. We use the representation (4.1) with $m=[(d-1) / 2]+1$. It is clear from (2.12), (2.52) and Lemma 3.5 that

$$
\frac{\partial^{m} \hat{G}_{\mathbf{a}}(\xi, \eta)}{\partial \eta^{m}}=\frac{g_{m}(\xi, \eta)}{\left[|\operatorname{Im}(\eta)|+\Lambda|e(\xi)|^{2}\right]},
$$

where the function $g_{m}$ is in $L_{w}^{(1+d / 2) / m}\left([-\pi, \pi]^{d+1}, \mathbf{C}\right)$ with norm $\left\|g_{m}\right\|_{(1+d / 2) / m, w}$ satisfying the inequality

$$
\left\|g_{m}\right\|_{(1+d / 2) / m, w} \leq C_{d} \Lambda^{-m d /(d+2)}\left(\frac{\Lambda}{\lambda}\right)^{3 d+4} .
$$

The constant $C_{d}$ in (4.3) depends only on $d$. We write the integral on the RHS of (4.1) as a sum over four regions $A, B, D, E$, where

$$
\begin{array}{ll}
A=\left\{t \Lambda|e(\xi)|^{2}>1,\right. & \left.|\operatorname{Im}(\eta)|<\Lambda|e(\xi)|^{2}\right\}, \\
B=\left\{t \Lambda|e(\xi)|^{2}>1,\right. & \left.|\operatorname{Im}(\eta)|>\Lambda|e(\xi)|^{2}\right\}, \\
D=\left\{t \Lambda|e(\xi)|^{2}<1,\right. & \left.|\operatorname{Im}(\eta)|<\Lambda|e(\xi)|^{2}\right\}, \\
E=\left\{t \Lambda|e(\xi)|^{2}<1,\right. & \left.|\operatorname{Im}(\eta)|>\Lambda|e(\xi)|^{2}\right\} .
\end{array}
$$

Now we have that

$$
\int_{A} d \xi d[\operatorname{Im}(\eta)] \frac{\left|g_{m}(\xi, \eta)\right|}{\left[|\operatorname{Im}(\eta)|+\Lambda|e(\xi)|^{2}\right]} \leq \sum_{r=0}^{\infty} \frac{t}{2^{r}} \int_{A_{r}} d \xi d[\operatorname{Im}(\eta)]\left|g_{m}(\xi, \eta)\right|,
$$

where

$$
A_{r}=\left\{2^{r}<t \Lambda|e(\xi)|^{2}<2^{r+1}, t|\operatorname{Im}(\eta)|<2^{r+1}\right\} .
$$

We use now the well-known fact that if $f \in L_{w}^{p}\left([-\pi, \pi]^{d+1}, \mathbf{C}\right), 1<p<\infty$, then for any measurable set $F$ one has

$$
\int_{F}|f| \leq C_{p}\|f\|_{p, w} m(F)^{1-1 / p},
$$


where the constant $C_{p}$ depends only on $p$. Hence, taking $p=(1+d / 2) / m$, we have from (4.3) that

$$
\int_{A_{r}} d \xi d[\operatorname{Im}(\eta)]\left|g_{m}(\xi, \eta)\right| \leq C_{d}\left(\frac{\Lambda}{\lambda}\right)^{3 d+4} \Lambda^{-d / 2}\left[\frac{2^{r}}{t}\right]^{1+d / 2-m}
$$

for some constant $C_{d}$ depending only on $d$. Since $d$ is odd, $m>d / 2$. Hence the series on the RHS of (4.5) converges, whence we obtain the inequality

$$
\frac{1}{t^{m}} \int_{A}\left|\frac{\partial^{m} \hat{G}_{\mathbf{a}}(\xi, \eta)}{\partial \eta^{m}}\right| d \xi d[\operatorname{Im}(\eta)] \leq C_{d}\left(\frac{\Lambda}{\lambda}\right)^{3 d+4} \frac{1}{(\Lambda t)^{d / 2}} .
$$

In the case of region $\mathrm{B}$, we have that

$$
\int_{B} d \xi d[\operatorname{Im}(\eta)] \frac{\left|g_{m}(\xi, \eta)\right|}{\left[|\operatorname{Im}(\eta)|+\Lambda|e(\xi)|^{2}\right]} \leq \sum_{r, k=0}^{\infty} \frac{t}{2^{r+k}} \int_{B_{r, k}} d \xi d[\operatorname{Im}(\eta)]\left|g_{m}(\xi, \eta)\right|,
$$

where

$$
B_{r, k}=\left\{2^{r}<t \Lambda|e(\xi)|^{2}<2^{r+1}, 2^{r+k}<t|\operatorname{Im}(\eta)|<2^{r+k+1}\right\} .
$$

From (4.7) we have that

$$
\int_{B_{r, k}} d \xi d[\operatorname{Im}(\eta)]\left|g_{m}(\xi, \eta)\right| \leq C_{d}\left(\frac{\Lambda}{\lambda}\right)^{3 d+4} \Lambda^{-d / 2}\left[\frac{2^{r}}{t}\right]^{1+d / 2-m} 2^{k(1-1 / p)},
$$

for a constant $C_{d}$ depending only on $d$. Again we see that the series on the RHS of (4.9) converges. We conclude that

$$
\frac{1}{t^{m}} \int_{B}\left|\frac{\partial^{m} \hat{G}_{\mathbf{a}}(\xi, \eta)}{\partial \eta^{m}}\right| d \xi d[\operatorname{Im}(\eta)] \leq C_{d}\left(\frac{\Lambda}{\lambda}\right)^{3 d+4} \frac{1}{(\Lambda t)^{d / 2}} .
$$

To deal with the region $D$ we consider the integral

$$
\int_{D} d \xi d[\operatorname{Im}(\eta)] \frac{|\operatorname{Im}(\eta)|\left|g_{m}(\xi, \eta)\right|}{\left[|\operatorname{Im}(\eta)|+\Lambda|e(\xi)|^{2}\right]} \leq \sum_{r=0}^{\infty} \int_{D_{r}} d \xi d[\operatorname{Im}(\eta)]\left|g_{m}(\xi, \eta)\right|
$$

where

$$
D_{r}=\left\{2^{-r-1}<t \Lambda|e(\xi)|^{2}<2^{-r}, t|\operatorname{Im}(\eta)|<2^{-r}\right\} .
$$

In view of (4.3), (4.7) we have that

$$
\int_{D_{r}} d \xi d[\operatorname{Im}(\eta)]\left|g_{m}(\xi, \eta)\right| \leq C_{d}\left(\frac{\Lambda}{\lambda}\right)^{3 d+4} \Lambda^{-d / 2}\left[\frac{2^{-r}}{t}\right]^{1+d / 2-m} .
$$

Since $m<1+d / 2$ the series on the RHS of (4.12) converges. We conclude that

$$
\frac{1}{t^{m-1}} \int_{D}\left|\frac{\partial^{m} \hat{G}_{\mathbf{a}}(\xi, \eta)}{\partial \eta^{m}}\right||\operatorname{Im}(\eta)| d \xi d[\operatorname{Im}(\eta)] \leq C_{d}\left(\frac{\Lambda}{\lambda}\right)^{3 d+4} \frac{1}{(\Lambda t)^{d / 2}}
$$

We deal with the region $E$ similarly. Thus we consider for any $\delta, 0 \leq \delta \leq 1$, the integral

$$
\begin{aligned}
\int_{E} d \xi d[\operatorname{Im}(\eta)] \frac{|\operatorname{Im}(\eta)|^{\delta}\left|g_{m}(\xi, \eta)\right|}{\left[|\operatorname{Im}(\eta)|+\Lambda|e(\xi)|^{2}\right]} \\
\leq \sum_{r, k=0}^{\infty}\left(\frac{t}{2^{-r-1+k}}\right)^{1-\delta} \int_{E_{r, k}} d \xi d[\operatorname{Im}(\eta)]\left|g_{m}(\xi, \eta)\right|,
\end{aligned}
$$


where

$$
E_{r, k}=\left\{2^{-r-1}<t \Lambda|e(\xi)|^{2}<2^{-r}, 2^{-r-1+k}<t|\operatorname{Im}(\eta)|<2^{-r+k}\right\} .
$$

From (4.3), (4.7) we have that

$$
\int_{E_{r, k}} d \xi d[\operatorname{Im}(\eta)]\left|g_{m}(\xi, \eta)\right| \leq C_{d}\left(\frac{\Lambda}{\lambda}\right)^{3 d+4} \Lambda^{-d / 2}\left[\frac{2^{-r}}{t}\right]^{1+d / 2-m} 2^{k(1-1 / p)} .
$$

If we choose $\delta$ to satisfy the inequality

$$
0 \leq m-\frac{d}{2}<\delta<m /(1+d / 2)<1,
$$

then the series on the RHS of 4.15) converges. We conclude that

$$
\frac{1}{t^{m-\delta}} \int_{E}\left|\frac{\partial^{m} \hat{G}_{\mathbf{a}}(\xi, \eta)}{\partial \eta^{m}}\right||\operatorname{Im}(\eta)|^{\delta} d \xi d[\operatorname{Im}(\eta)] \leq C_{d}\left(\frac{\Lambda}{\lambda}\right)^{3 d+4} \frac{1}{(\Lambda t)^{d / 2}} .
$$

The inequality (4.2) for $\Lambda t \geq 1$ follows now from (4.8), (4.11), (4.14), (4.18), by letting $\operatorname{Re}(\eta) \rightarrow 0$ in (4.1).

Lemma 4.2. Suppose $d$ is even, $4 d \Lambda \leq 1$ and $x \in \mathbf{Z}^{d}, t \in \mathbf{Z}, t \geq 0$. Then there is a positive constant $C_{d}$, depending only on $d$, such that

$$
\left|G_{\mathbf{a}}(x, t)\right| \leq \frac{C_{d}(\Lambda / \lambda)^{3 d+6}}{1+(\Lambda t)^{d / 2}} .
$$

Proof. We can see similarly to the derivation of (4.1) that for any integer $m \geq 1$ one has

$$
\begin{aligned}
(2 \pi)^{d+1} G_{\mathbf{a}}(x, t)=\frac{1}{2} \frac{(-1)^{m+1}}{t^{m}} \int_{[-\pi, \pi]^{d}} d \xi e^{-i x \cdot \xi} \int_{-\pi}^{\pi} d[\operatorname{Im}(\eta)] \\
\left\{e^{\eta t}-1\right\}\left\{\frac{\partial^{m} \hat{G}_{\mathbf{a}}(\xi, \eta+\pi i / t)}{\partial \eta^{m}}-\frac{\partial^{m} \hat{G}_{\mathbf{a}}(\xi, \eta)}{\partial \eta^{m}}\right\} .
\end{aligned}
$$

We take $m=[(d-1) / 2]+1$ in (4.20). It follows from (2.12), (2.52) and Lemmas $3.5,3.6$ that

$$
\frac{\partial^{m} \hat{G}_{\mathbf{a}}(\xi, \eta+i \varepsilon)}{\partial \eta^{m}}-\frac{\partial^{m} \hat{G}_{\mathbf{a}}(\xi, \eta)}{\partial \eta^{m}}=\frac{|\varepsilon|^{\alpha} g_{m, \alpha, \varepsilon}(\xi, \eta)}{\left[|\operatorname{Im}(\eta)|+\Lambda|e(\xi)|^{2}\right]}
$$

where the function $g_{m, \alpha, \varepsilon}$ is in $L^{(1+d / 2) /(m+\alpha)}\left([-\pi, \pi]^{d+1}\right.$, C) for $\alpha \geq 0$ satisfying $m+\alpha<1+d / 2$. There is a constant $C_{d, \alpha}$ depending only on $d$ and $\alpha$ such that

$$
\left\|g_{m, \alpha, \varepsilon}\right\|_{(1+d / 2) /(m+\alpha), w} \leq C_{d, \alpha} \Lambda^{-(m+\alpha) d /(d+2)}\left(\frac{\Lambda}{\lambda}\right)^{3 d+6} .
$$

We proceed now exactly as in Lemma 4.1, choosing $\alpha>0$ and $\delta$ to satisfy (4.17) with $m$ replaced by $m+\alpha$. It is easy to see from 4.21, 4.22 that the integrals over $A, B, D, E$ corresponding to the representation (4.20) all converge. Hence we obtain the inequality (4.19).

Lemma 4.3. Suppose $4 d \Lambda \leq 1$ and $x \in \mathbf{Z}^{d}, t \in \mathbf{Z}, t \geq 0$. Then there is a positive constant $C_{d}$, depending only on $d$, such that

$$
\left|\nabla G_{\mathbf{a}}(x, t)\right| \leq \frac{C_{d}(\Lambda / \lambda)^{3 d+6}}{1+(\Lambda t)^{d / 2+1 / 2}} .
$$


Let $\gamma$ satisfy $0 \leq \gamma<1$. Then there is a constant $C_{d, \gamma}$ depending only on $d, \gamma$ such that

$$
\left|\nabla \nabla G_{\mathbf{a}}(x, t)\right| \leq \frac{C_{d, \gamma}(\Lambda / \lambda)^{3 d+6}}{1+(\Lambda t)^{d / 2+\gamma}} .
$$

Proof. We use the representation (4.20). Defining the regions $A$ and $A_{r}$ by (4.4), (4.6) we have that

$$
\begin{aligned}
\int_{A} d \xi d[\operatorname{Im}(\eta)] \frac{|e(\xi)|\left|g_{m, \alpha, \varepsilon}(\xi, \eta)\right|}{\left[|\operatorname{Im}(\eta)|+\Lambda|e(\xi)|^{2}\right]} & \\
& \leq \Lambda^{-\frac{1}{2}} \sum_{r=0}^{\infty}\left(\frac{t}{2^{r}}\right)^{1 / 2} \int_{A_{r}} d \xi d[\operatorname{Im}(\eta)]\left|g_{m, \alpha, \varepsilon}(\xi, \eta)\right| .
\end{aligned}
$$

From (4.22) we have that

$$
\int_{A_{r}} d \xi d[\operatorname{Im}(\eta)]\left|g_{m, \alpha, \varepsilon}(\xi, \eta)\right| \leq C_{d, \alpha}\left(\frac{\Lambda}{\lambda}\right)^{3 d+6} \Lambda^{-d / 2}\left[\frac{2^{r}}{t}\right]^{1+d / 2-m-\alpha}
$$

The series on the RHS of (4.25) converges if $\alpha>0$ in the case of $d$ odd and $\alpha>1 / 2$ in the case of $d$ even. Choosing $\alpha$ appropriately, we conclude there is a constant $C_{d}$, depending only on $d$, such that

$$
\begin{aligned}
& \frac{1}{t^{m}} \int_{A}|e(\xi)|\left|\frac{\partial^{m} \hat{G}_{\mathbf{a}}(\xi, \eta+\pi i / t)}{\partial \eta^{m}}-\frac{\partial^{m} \hat{G}_{\mathbf{a}}(\xi, \eta)}{\partial \eta^{m}}\right| d \xi d[\operatorname{Im}(\eta)] \\
& \quad \leq C_{d}\left(\frac{\Lambda}{\lambda}\right)^{3 d+6} \frac{1}{(\Lambda t)^{d / 2+1 / 2}} .
\end{aligned}
$$

Defining the regions $B$ and $B_{r, k}$ by (4.4), (4.10) we have that

$$
\begin{aligned}
\int_{B} d \xi d[\operatorname{Im}(\eta)] \frac{|e(\xi)|\left|g_{m, \alpha, \varepsilon}(\xi, \eta)\right|}{\left[|\operatorname{Im}(\eta)|+\Lambda|e(\xi)|^{2}\right]} \\
\quad \leq \Lambda^{-\frac{1}{2}} \sum_{r, k=0}^{\infty}\left(\frac{2^{r+1}}{t}\right)^{1 / 2} \frac{t}{2^{r+k}} \int_{B_{r}, k} d \xi d[\operatorname{Im}(\eta)]\left|g_{m, \alpha, \varepsilon}(\xi, \eta)\right| .
\end{aligned}
$$

From (4.22), 4.7) we have that

$$
\begin{aligned}
& \int_{B_{r, k}} d \xi d[\operatorname{Im}(\eta)]\left|g_{m, \alpha, \varepsilon}(\xi, \eta)\right| \\
& \quad \leq C_{d, \alpha}\left(\frac{\Lambda}{\lambda}\right)^{3 d+6} \Lambda^{-d / 2}\left[\frac{2^{r}}{t}\right]^{1+d / 2-m-\alpha} 2^{k(1-1 / p)}
\end{aligned}
$$

where $p=(1+d / 2) /(m+\alpha)$. The series on the RHS of (4.28) converges provided $\alpha>0$ in the case of $d$ odd and $\alpha>1 / 2$ in the case of $d$ even. We conclude there is a constant $C_{d}$, depending only on $d$, such that

$$
\begin{aligned}
& \frac{1}{t^{m}} \int_{B}|e(\xi)| \frac{\partial^{m} \hat{G}_{\mathbf{a}}(\xi, \eta+\pi i / t)}{\partial \eta^{m}}-\frac{\partial^{m} \hat{G}_{\mathbf{a}}(\xi, \eta)}{\partial \eta^{m}} \mid \\
& \leq C_{d}\left(\frac{\Lambda}{\lambda}\right)^{3 d+6} \frac{1}{(\Lambda t)^{d / 2+1 / 2}}
\end{aligned}
$$


Defining the regions $D$ and $D_{r}$ by (4.4), (4.13) we have that

$$
\begin{aligned}
\int_{D} d \xi d[\operatorname{Im}(\eta)] \frac{|e(\xi)||\operatorname{Im}(\eta)|\left|g_{m, \alpha, \varepsilon}(\xi, \eta)\right|}{\left[|\operatorname{Im}(\eta)|+\Lambda|e(\xi)|^{2}\right]} \\
\leq \Lambda^{-\frac{1}{2}} \sum_{r=0}^{\infty}\left(\frac{2^{-r}}{t}\right)^{1 / 2} \int_{D_{r}} d \xi d[\operatorname{Im}(\eta)]\left|g_{m, \alpha, \varepsilon}(\xi, \eta)\right| .
\end{aligned}
$$

From (4.22), (4.7) we have that

$$
\begin{array}{rl}
\int_{D_{r}} & d \xi d[\operatorname{Im}(\eta)]\left|g_{m, \alpha, \varepsilon}(\xi, \eta)\right| \\
& \leq C_{d, \alpha}\left(\frac{\Lambda}{\lambda}\right)^{3 d+6} \Lambda^{-d / 2}\left[\frac{2^{-r}}{t}\right]^{1+d / 2-m-\alpha} .
\end{array}
$$

Taking $\alpha=0$ in the last inequality, we see that the series on the RHS of (4.31) converges.

We conclude that there is a constant $C_{d}$, depending only on $d$, such that

$$
\begin{aligned}
\frac{1}{t^{m-1}} \int_{D}|e(\xi)||\operatorname{Im}(\eta)| \mid \frac{\partial^{m} \hat{G}_{\mathbf{a}}(\xi, \eta+\pi i / t)}{\partial \eta^{m}} & -\frac{\partial^{m} \hat{G}_{\mathbf{a}}(\xi, \eta)}{\partial \eta^{m}} \mid d \xi d[\operatorname{Im}(\eta)] \\
& \leq C_{d}\left(\frac{\Lambda}{\lambda}\right)^{3 d+6} \frac{1}{(\Lambda t)^{d / 2+1 / 2}}
\end{aligned}
$$

Defining the regions $E$ and $E_{r, k}$ by (4.4), (4.16) we have for any $\delta, 0 \leq \delta \leq 1$, the inequality

$$
\begin{aligned}
& \int_{E} d \xi d[\operatorname{Im}(\eta)] \frac{|e(\xi)||\operatorname{Im}(\eta)|^{\delta}\left|g_{m, \alpha, \varepsilon}(\xi, \eta)\right|}{\left[|\operatorname{Im}(\eta)|+\Lambda|e(\xi)|^{2}\right]} \\
& \leq \Lambda^{-\frac{1}{2}} \sum_{r, k=0}^{\infty}\left(\frac{2^{-r}}{t}\right)^{1 / 2}\left(\frac{t}{2^{-r-1+k}}\right)^{1-\delta} \int_{E_{r}, k} d \xi d[\operatorname{Im}(\eta)]\left|g_{m, \alpha, \varepsilon}(\xi, \eta)\right|
\end{aligned}
$$

From (4.22), (4.7) we have that

$$
\begin{array}{rl}
\int_{E_{r, k}} & d \xi d[\operatorname{Im}(\eta)]\left|g_{m, \alpha, \varepsilon}(\xi, \eta)\right| \\
\leq & C_{d}\left(\frac{\Lambda}{\lambda}\right)^{3 d+6} \Lambda^{-d / 2}\left[\frac{2^{-r}}{t}\right]^{1+d / 2-m-\alpha} 2^{k(1-1 / p)},
\end{array}
$$

where $p=(1+d / 2) /(m+\alpha)$. If we choose $\alpha=0$ and $\delta$ to satisfy (4.17), the series on the RHS of 4.33) converges. We conclude there is a constant $C_{d}$, depending only on $d$, such that

$$
\begin{aligned}
& \frac{1}{t^{m-\delta}} \int_{E}|e(\xi)||\operatorname{Im}(\eta)|^{\delta} \mid \frac{\partial^{m} \hat{G}_{\mathbf{a}}(\xi, \eta+\pi i / t)}{\partial \eta^{m}}-\frac{\partial^{m} \hat{G}_{\mathbf{a}}(\xi, \eta)}{\partial \eta^{m}} \mid d \xi d[\operatorname{Im}(\eta)] \\
& \leq C_{d}\left(\frac{\Lambda}{\lambda}\right)^{3 d+6} \frac{1}{(\Lambda t)^{d / 2}+1 / 2}
\end{aligned}
$$

The inequality (4.23) follows now from the representation (4.20) and the inequalities (4.27), (4.30), (4.32), (4.35). The inequality (4.24) follows by similar argument. We obtain estimates similar to (4.27), (4.30), (4.32), (4.35) but with $|e(\xi)|$ replaced by 
$|e(\xi)|^{2 \gamma}$ in the integrand. The estimates on the integrals over the regions $A$ and $B$ diverge as $\gamma \rightarrow 1$.

Lemma 4.4. Suppose $4 d \Lambda \leq 1$ and $x \in \mathbf{Z}^{d}, t \in \mathbf{Z}, t \geq 0$. Let $\gamma$ satisfy $0 \leq \gamma<1$. Then there is a positive constant $C_{d, \gamma}$, depending only on $d, \gamma$, such that

$$
\left|G_{\mathbf{a}}(x, t+1)-G_{\mathbf{a}}(x, t)\right| \leq \frac{\Lambda^{\gamma} C_{d, \gamma}(\Lambda / \lambda)^{3 d+6}}{1+(\Lambda t)^{d / 2+\gamma}} .
$$

Proof. The inequality (4.36) is a consequence of Lemma 2.11 if $\Lambda t \leq 1$, so we shall assume $\Lambda t>1$. It follows from (2.2) that for any integer $m \geq 1$ one has

$$
\begin{gathered}
(2 \pi)^{d+1}\left[G_{\mathbf{a}}(x, t+1)-G_{\mathbf{a}}(x, t)\right]=\frac{1}{2} \frac{(-1)^{m+1}}{t^{m}} \int_{[-\pi, \pi]^{d}} d \xi e^{-i x \cdot \xi} \int_{-\pi}^{\pi} d[\operatorname{Im}(\eta)] \\
\left\{e^{\eta}-1\right\} e^{\eta t}\left\{\frac{\partial^{m} \hat{G}_{\mathbf{a}}(\xi, \eta+\pi i / t)}{\partial \eta^{m}}-\frac{\partial^{m} \hat{G}_{\mathbf{a}}(\xi, \eta)}{\partial \eta^{m}}\right\} \\
+\frac{1}{4} \frac{(-1)^{m+1}}{t^{m}} \int_{[-\pi, \pi]^{d}} d \xi e^{-i x \cdot \xi} \int_{-\pi}^{\pi} d[\operatorname{Im}(\eta)]\left\{e^{\pi i / t}-1\right\}\left\{e^{\eta(t+1)}-1\right\} \\
\left\{\frac{\partial^{m} \hat{G}_{\mathbf{a}}(\xi, \eta+\pi i / t)}{\partial \eta^{m}}-\frac{\partial^{m} \hat{G}_{\mathbf{a}}(\xi, \eta+\pi i / t-\pi i /(t+1))}{\partial \eta^{m}}\right\} .
\end{gathered}
$$

It is clear by the argument of Lemma 4.2 that the second integral in (4.37) is bounded by the RHS of (4.36) with $\gamma=1$. We shall concentrate therefore on the first integral in (4.37).

Defining the regions $A$ and $A_{r}$ by (4.4), (4.6) we have that

$$
\begin{aligned}
& \int_{A} d \xi d[\operatorname{Im}(\eta)] \frac{|\operatorname{Im}(\eta)|^{\gamma}\left|g_{m, \alpha, \varepsilon}(\xi, \eta)\right|}{[|\operatorname{Im}(\eta)|}+\left.\Lambda|e(\xi)|^{2}\right] \\
& \leq \sum_{r=0}^{\infty}\left(\frac{t}{2^{r}}\right)^{1-\gamma} \int_{A_{r}} d \xi d[\operatorname{Im}(\eta)]\left|g_{m, \alpha, \varepsilon}(\xi, \eta)\right| .
\end{aligned}
$$

From (4.26) we see that the series in (4.38) converges provided $m+\alpha-d / 2>\gamma$. Choosing $\alpha$ appropriately, we see there is a constant $C_{d, \gamma}$, depending only on $d$ and $\gamma<1$, such that

$$
\begin{aligned}
& \frac{1}{t^{m}} \int_{A}|\operatorname{Im}(\eta)|^{\gamma}\left|\frac{\partial^{m} \hat{G}_{\mathbf{a}}(\xi, \eta+\pi i / t)}{\partial \eta^{m}}-\frac{\partial^{m} \hat{G}_{\mathbf{a}}(\xi, \eta)}{\partial \eta^{m}}\right| d \xi d[\operatorname{Im}(\eta)] \\
& \leq C_{d, \gamma}\left(\frac{\Lambda}{\lambda}\right)^{3 d+6} \frac{1}{t^{\gamma}(\Lambda t)^{d / 2}}
\end{aligned}
$$

Defining the regions $B$ and $B_{r, k}$ by (4.4), (4.10) we have that

$$
\begin{aligned}
& \int_{B} d \xi d[\operatorname{Im}(\eta)] \frac{|\operatorname{Im}(\eta)|^{\gamma}\left|g_{m, \alpha, \varepsilon}(\xi, \eta)\right|}{\left[|\operatorname{Im}(\eta)|+\Lambda|e(\xi)|^{2}\right]} \\
& \leq \sum_{r, k=0}^{\infty}\left[\frac{t}{2^{r+k}}\right]^{1-\gamma} \int_{B_{r, k}} d \xi d[\operatorname{Im}(\eta)]\left|g_{m, \alpha, \varepsilon}(\xi, \eta)\right| .
\end{aligned}
$$


From (4.29) we see that the series in (4.40) converges provided $\gamma<m+\alpha-d / 2$ and $\gamma<(m+\alpha) /(1+d / 2)$. Choosing $\alpha$ appropriately, we see there is a constant $C_{d, \gamma}$, depending only on $d$ and $\gamma<1$, such that

$$
\begin{aligned}
& \frac{1}{t^{m}} \int_{B}|\operatorname{Im}(\eta)|^{\gamma}\left|\frac{\partial^{m} \hat{G}_{\mathbf{a}}(\xi, \eta+\pi i / t)}{\partial \eta^{m}}-\frac{\partial^{m} \hat{G}_{\mathbf{a}}(\xi, \eta)}{\partial \eta^{m}}\right| d \xi d[\operatorname{Im}(\eta)] \\
& \leq C_{d, \gamma}\left(\frac{\Lambda}{\lambda}\right)^{3 d+6} \frac{1}{t^{\gamma}(\Lambda t)^{d / 2}}
\end{aligned}
$$

Defining $D$ by (4.4) we see from the argument of Lemma 4.2 that

$$
\begin{aligned}
& \frac{1}{t^{m}} \int_{D}|\operatorname{Im}(\eta)| \frac{\partial^{m} \hat{G}_{\mathbf{a}}(\xi, \eta+\pi i / t)}{\partial \eta^{m}}-\frac{\partial^{m} \hat{G}_{\mathbf{a}}(\xi, \eta)}{\partial \eta^{m}} \mid d \xi d[\operatorname{Im}(\eta)] \\
& \leq C_{d}\left(\frac{\Lambda}{\lambda}\right)^{3 d+6} \frac{1}{t(\Lambda t)^{d / 2}}
\end{aligned}
$$

for some constant $C_{d}$ depending only on $d$. Defining the regions $E$ and $E_{r, k}$ by (4.4), (4.16) we have that

$$
\begin{aligned}
\int_{E} d \xi d[\operatorname{Im}(\eta)] & \frac{|\operatorname{Im}(\eta)|^{\gamma}\left|g_{m, \alpha, \varepsilon}(\xi, \eta)\right|}{\left[|\operatorname{Im}(\eta)|+\Lambda|e(\xi)|^{2}\right]} \\
& \leq \sum_{r, k=0}^{\infty}\left(\frac{t}{2^{-r-1+k}}\right)^{1-\gamma} \int_{E_{r, k}} d \xi d[\operatorname{Im}(\eta)]\left|g_{m, \alpha, \varepsilon}(\xi, \eta)\right| .
\end{aligned}
$$

It follows from (4.34) that the series on the RHS of (4.43) converges provided $\gamma$ satisfies the inequality

$$
m+\alpha-d / 2<\gamma<(m+\alpha) /(1+d / 2) .
$$

Choosing $\alpha$ appropriately, we see there is a constant $C_{d, \gamma}$, depending only on $d$ and $\gamma<1$, such that

$$
\begin{aligned}
& \frac{1}{t^{m}} \int_{E}|\operatorname{Im}(\eta)|^{\gamma}\left|\frac{\partial^{m} \hat{G}_{\mathbf{a}}(\xi, \eta+\pi i / t)}{\partial \eta^{m}}-\frac{\partial^{m} \hat{G}_{\mathbf{a}}(\xi, \eta)}{\partial \eta^{m}}\right| d \xi d[\operatorname{Im}(\eta)] \\
& \leq C_{d, \gamma}\left(\frac{\Lambda}{\lambda}\right)^{3 d+6} \frac{1}{t^{\gamma}(\Lambda t)^{d / 2}}
\end{aligned}
$$

It follows now from (4.39), (4.41), (4.42), 4.44) that the first integral in (4.37) is bounded by the RHS of (4.36).

Evidently Lemmas 4.1-4.4 imply Theorem 1.1 except for the exponential fall off. We obtain the exponential fall off by analytically continuing the function $q(\xi, \eta)$ of (2.13), (2.24). We have the following extension of Lemma 2.4.

Lemma 4.5. Suppose $4 d \Lambda \leq 1$. Then the function $q(\xi, \eta), \xi \in \mathbf{R}^{d}, \operatorname{Re}(\eta)>0$, extends analytically to the region $\mathbf{e}_{1} \cdot \xi \in \mathbf{C}$ satisfying

$$
e^{\operatorname{Re}(\eta)}-1>2 \Lambda[\cosh \theta+3][\cosh \theta-1], \quad \theta=\operatorname{Im}\left(\mathbf{e}_{1} \cdot \xi\right) .
$$

Proof. We first establish for $\psi \in L^{2}(\Omega)$ the a priori bound,

$$
\begin{aligned}
& \mid\left\langle\overline{\psi\left(\tau_{0,1} \cdot\right)}\left[I-\Lambda \partial_{\xi}^{*} \partial_{\xi}\right] \psi(\cdot)\right\rangle+\frac{\Lambda}{2}\left[\left\langle\left|\partial_{\xi} \psi\right|^{2}\right\rangle+\left\langle\left|\partial_{\bar{\xi}} \psi\right|^{2}\right\rangle\right] \\
\leq & \left\langle|\psi|^{2}\right\rangle+2 \Lambda[\cosh \theta+3][\cosh \theta-1]\left\langle|\psi|^{2}\right\rangle
\end{aligned}
$$


where we are assuming $\mathbf{e}_{k} \cdot \xi \in \mathbf{R}, k>1$, and $\mathbf{e}_{1} \cdot \xi \in \mathbf{C}$ with $\operatorname{Im}\left(\mathbf{e}_{1} \cdot \xi\right)=\theta$. To see this let $\rho>0$ be defined by

$$
\rho=\sum_{j=2}^{d}\left\langle\left|\partial_{j, \xi} \psi\right|^{2}\right\rangle /\left\langle|\psi|^{2}\right\rangle,
$$

where $\partial_{j, \xi}$ is given by (2.4). Evidently $\rho \leq 4(d-1)$. Since $4 d \Lambda \leq 1$, we have that

$$
\begin{aligned}
& \left|\left\langle\overline{\psi\left(\tau_{0,1} \cdot\right)}\left[I-\Lambda \partial_{\xi}^{*} \partial_{\xi}\right] \psi(\cdot)\right\rangle\right| \\
& \quad \leq[1-\Lambda \rho-4 \Lambda]\left\langle|\psi|^{2}\right\rangle+\Lambda\left|\left\langle\overline{\psi\left(\tau_{0,1} \cdot\right)}\left[4 I-\partial_{1, \xi}^{*} \partial_{1, \xi}\right] \psi(\cdot)\right\rangle\right| .
\end{aligned}
$$

From (2.4) we have that

$$
\begin{aligned}
& \left\langle\overline{\psi\left(\tau_{0,1} \cdot\right)}\left[4 I-\partial_{1, \xi}^{*} \partial_{1, \xi}\right] \psi(\cdot)\right\rangle \\
= & \left\langle\overline{\psi\left(\tau_{0,1} \cdot\right)}\left[2 I+\partial_{1, \xi}^{*}\right]\left[2 I+\partial_{1, \xi}\right] \psi(\cdot)\right\rangle \\
= & \left\langle\left\{\overline{\left[2 I+\partial_{1, \bar{\xi}}\right] \psi\left(\tau_{0,1} \cdot\right)}\right\}\left\{\left[2 I+\partial_{1, \xi}\right] \psi(\cdot)\right\}\right\rangle .
\end{aligned}
$$

We conclude that

$$
\begin{aligned}
\left|\left\langle\overline{\psi\left(\tau_{0,1} \cdot\right)}\left[4 I-\partial_{1, \xi}^{*} \partial_{1, \xi}\right] \psi(\cdot)\right\rangle\right| & \\
& \leq\left\langle\left|\left[2 I+\partial_{1, \bar{\xi}}\right] \psi(\cdot)\right|^{2}\right\rangle^{1 / 2}\left\langle\left|\left[2 I+\partial_{1, \xi}\right] \psi(\cdot)\right|^{2}\right\rangle^{1 / 2} .
\end{aligned}
$$

Let us write

$$
\exp \left[-i \operatorname{Re}\left(\mathbf{e}_{1} \cdot \xi\right)\right]\left\langle\overline{\psi(\cdot)} \psi\left(\tau_{\mathbf{e}_{1,0}} \cdot\right)\right\rangle /\left\langle|\psi|^{2}\right\rangle=r e^{i \beta},
$$

where $r>0, \beta \in \mathbf{R}$. Evidently one has $r \leq 1$. Then we have

$$
\left\langle\left|\left[2 I+\partial_{1, \xi}\right] \psi(\cdot)\right|^{2}\right\rangle=\left[1+e^{2 \theta}+2 e^{\theta} r \cos \beta\right]\left\langle|\psi|^{2}\right\rangle .
$$

Similarly, we have

$$
\left\langle\left|\left[2 I+\partial_{1, \bar{\xi}}\right] \psi(\cdot)\right|^{2}\right\rangle=\left[1+e^{-2 \theta}+2 e^{-\theta} r \cos \beta\right]\left\langle|\psi|^{2}\right\rangle .
$$

From (4.48) and the last two identities we conclude that

$$
\left|\left\langle\overline{\psi\left(\tau_{0,1} \cdot\right)} \mid\left[4 I-\partial_{1, \xi}^{*} \partial_{1, \xi}\right] \psi(\cdot)\right\rangle\right| \leq 2[\cosh \theta+r \cos \beta]\left\langle|\psi|^{2}\right\rangle .
$$

Next we consider the second term on the LHS of (4.46). We have

$$
\begin{aligned}
\left\langle\left|\partial_{\xi} \psi\right|^{2}\right\rangle & =\left[\rho+1+e^{2 \theta}-2 e^{\theta} r \cos \beta\right]\left\langle|\psi|^{2}\right\rangle, \\
\left\langle\left|\partial_{\bar{\xi}} \psi\right|^{2}\right\rangle & =\left[\rho+1+e^{-2 \theta}-2 e^{-\theta} r \cos \beta\right]\left\langle|\psi|^{2}\right\rangle .
\end{aligned}
$$

Hence we have

$$
\frac{1}{2}\left[\left\langle\left|\partial_{\xi} \psi\right|^{2}\right\rangle+\left\langle\left|\partial_{\bar{\xi}} \psi\right|^{2}\right\rangle=[\rho+1+\cosh 2 \theta-2 \cosh \theta r \cos \beta]\left\langle|\psi|^{2}\right\rangle .\right.
$$

The inequality (4.46) follows now from this last inequality, (4.47) and (4.49).

Let $Q$ be the bilinear form on $L^{2}(\Omega)$ defined by

$$
Q\left(\psi^{\prime}, \psi\right)=\left\langle\overline{\psi^{\prime}\left(\tau_{0,1} \cdot\right)}\left[e^{\eta} \psi\left(\tau_{0,1} \cdot\right)-\psi(\cdot)+\Lambda \partial_{\xi}^{*} \partial_{\xi} \psi(\cdot)\right]\right\rangle .
$$


Evidently there is a constant $A$ depending on $\eta, \xi$ such that $\left|Q\left(\psi^{\prime}, \psi\right)\right| \leq A\left\|\psi^{\prime}\right\|\|\psi\|$. From (4.46) we can see that if $\mathbf{e}_{k} \cdot \xi \in \mathbf{R}, k>1$, and $\mathbf{e}_{1} \cdot \xi, \eta \in \mathbf{C}$ satisfy (4.45), then

$$
e^{-i \operatorname{Im}(\eta)} Q(\psi, \psi) \geq \delta\|\psi\|^{2},
$$

for some $\delta>0$. It follows from the Lax-Milgram theorem that if $\varphi \in L^{2}(\Omega)$, then the equation

$$
e^{\eta} \psi\left(\tau_{0,1} \omega\right)-\psi(\omega)+\Lambda \partial_{\xi}^{*} \partial_{\xi} \psi(\omega)=\varphi(\omega), \quad \omega \in \Omega,
$$

is uniquely solvable for $\psi \in L^{2}(\Omega)$. Hence the operator $T_{\Lambda, \eta, \xi}$ defined by (2.19), (2.20) continues to be well defined provided $\mathbf{e}_{1} \cdot \xi, \eta$ satisfy (4.45). It further follows from (4.46) that $T_{\Lambda, \eta, \xi}$ as an operator on $\mathcal{H}(\Omega)$ has norm satisfying $\left\|T_{\Lambda, \eta, \xi}\right\| \leq 1$. It is also easy to see that the mapping $(\eta, \xi) \rightarrow T_{\Lambda, \eta, \xi}$ from $\mathbf{C}^{2} \times \mathbf{R}^{d-1}$ to the bounded linear operators on $\mathcal{H}(\Omega)$ is analytic in $\eta, \mathbf{e}_{1} \cdot \xi$ in the region (4.45). The analyticity of $q(\xi, \eta)$ follows from this and the representations (2.24), (2.31).

Next we extend Lemma 2.7 to complex $\mathbf{e}_{1} \cdot \xi$.

Lemma 4.6. Suppose $2 d \Lambda \leq 1, \mathbf{e}_{k} \cdot \xi \in \mathbf{R}, k>1$, $\mathbf{e}_{1} \cdot \xi, \eta \in \mathbf{C}$ satisfying

$$
e^{\operatorname{Re}(\eta)}-1>\Lambda[\cosh \theta+3][\cosh \theta-1], \quad \theta=\operatorname{Im}\left(\mathbf{e}_{1} \cdot \xi\right) .
$$

Then for any column vector $\rho \in \mathbf{C}^{d}$ with adjoint $\rho^{*} \in \mathbf{C}^{d}$ there are the inequalities

$$
0 \leq \operatorname{Re}\left[\rho^{*} q(\xi, \eta) \rho\right] \leq \Lambda|\rho|^{2},
$$

$$
\left|\operatorname{Im}\left[\rho^{*} q(\xi, \eta) \rho\right]\right| \leq \Lambda|\rho|^{2} .
$$

Proof. By Lemma 4.5 the solution $\Phi(\xi, \eta, \omega)$ of (2.11) exists for $(\xi, \eta)$ in the region (4.45). In the following we shall only need to assume that $(\xi, \eta)$ is in the larger region (4.50). Defining $\varphi(\xi, \eta, \omega)$ as in Lemma 2.7, we see that

$$
\rho^{*} q(\xi, \eta) \rho=\rho^{*}\langle\mathbf{a}(\cdot)\rangle \rho+\rho^{*}\left\langle\mathbf{a}(\cdot) \partial_{\xi} \varphi(\xi, \eta, \cdot)\right\rangle
$$

Let $\theta^{\prime}=\operatorname{Im}(\eta)$ and multiply $(\underline{2.42})$ by $e^{-i \theta^{\prime}} \overline{\varphi\left(\xi, \eta, \tau_{0,1} \omega\right)}$ and take the expectation. This yields the identity

$$
\begin{aligned}
& -\left\langle\left[\partial_{\xi}^{*} \mathbf{a}(\cdot) \rho\right] e^{-i \theta^{\prime}} \overline{\varphi\left(\xi, \eta, \tau_{0,1} \cdot\right)}\right\rangle \\
& =e^{\operatorname{Re}(\eta)}\left\langle|\varphi(\xi, \eta, \cdot)|^{2}\right\rangle-\left\langle\varphi(\xi, \eta, \cdot) e^{-i \theta^{\prime}} \overline{\varphi\left(\xi, \eta, \tau_{0,1} \cdot\right)}\right\rangle \\
& +\left\langle e^{-i \theta^{\prime}} \overline{\partial_{\xi} \varphi\left(\xi, \eta, \tau_{0,1} \cdot\right)} \mathbf{a}(\cdot) \partial_{\xi} \varphi(\xi, \eta, \cdot)\right\rangle .
\end{aligned}
$$

We define positive numbers $A$ and $B$ by

$$
\begin{aligned}
A^{2}= & \frac{1}{4}\left\langle\left[\overline{\partial_{\xi} \varphi(\xi, \eta, \cdot)+e^{i \theta^{\prime}} \partial_{\bar{\xi}} \varphi\left(\xi, \eta, \tau_{0,1} \cdot\right)}\right]\right. \\
& \left.\mathbf{a}(\cdot)\left[\partial_{\xi} \varphi(\xi, \eta, \cdot)+e^{i \theta^{\prime}} \partial_{\bar{\xi}} \varphi\left(\xi, \eta, \tau_{0,1} \cdot\right)\right]\right\rangle, \\
B^{2}= & \frac{1}{4}\left\langle\left[\overline{\partial_{\xi} \varphi(\xi, \eta, \cdot)-e^{i \theta^{\prime}} \partial_{\bar{\xi}} \varphi\left(\xi, \eta, \tau_{0,1} \cdot\right)}\right]\right. \\
& {\left.\left[\Lambda I_{d}-\mathbf{a}(\cdot)\right]\left[\partial_{\xi} \varphi(\xi, \eta, \cdot)-e^{i \theta^{\prime}} \partial_{\bar{\xi}} \varphi\left(\xi, \eta, \tau_{0,1} \cdot\right)\right]\right\rangle . }
\end{aligned}
$$


From (4.53) we then have the identity

$$
\begin{aligned}
& -\operatorname{Re} \rho^{*}\left\langle\mathbf{a}(\cdot) e^{i \theta^{\prime}} \partial_{\bar{\xi}} \varphi\left(\xi, \eta, \tau_{0,1} \cdot\right)\right\rangle=e^{\operatorname{Re}(\eta)}\left\langle|\varphi(\xi, \eta, \cdot)|^{2}\right\rangle \\
& -\operatorname{Re} e^{-i \theta^{\prime}}\left\langle\overline{\varphi\left(\xi, \eta, \tau_{0,1} \cdot\right)}\left[I-\frac{\Lambda}{2} \partial_{\xi}^{*} \partial_{\xi}\right] \varphi(\xi, \eta, \cdot)\right\rangle \\
& -\frac{\Lambda}{4}\left[\left\langle\left|\partial_{\xi} \varphi(\xi, \eta, \cdot)\right|^{2}\right\rangle+\left\langle\left|\partial_{\bar{\xi}} \varphi(\xi, \eta, \cdot)\right|^{2}\right\rangle\right]+A^{2}+B^{2} .
\end{aligned}
$$

In view of the inequality (4.46) with $\Lambda$ replaced by $\Lambda / 2$ we see from the last equation that if $(\xi, \eta)$ satisfies (4.50), then there is the inequality

$$
A^{2}+B^{2} \leq-\operatorname{Re} \rho^{*}\left\langle\mathbf{a}(\cdot) e^{i \theta^{\prime}} \partial_{\bar{\xi}} \varphi\left(\xi, \eta, \tau_{0,1} \cdot\right)\right\rangle .
$$

Arguing now as in Lemma 2.7 we conclude that

$$
A^{2}+B^{2} \leq \Lambda|\rho|^{2} .
$$

The remainder of the proof of (4.51), (4.52) follows from (4.56) in exactly the same way as in Lemma 2.7.

Remark 6. Lemma 4.6 suggests that $q(\xi, \eta)$ is analytic in the region 4.50) and that we need only assume $2 d \Lambda \leq 1$.

We extend Lemma 2.8 to complex $\mathbf{e}_{1} \cdot \xi$.

Lemma 4.7. Suppose $2 d \Lambda \leq 1, \mathbf{e}_{k} \cdot \xi \in \mathbf{R}, k>1$, $\mathbf{e}_{1} \cdot \xi, \eta \in \mathbf{C}$ satisfying (4.50). Then for any column vector $\rho \in \mathbf{C}^{d}$ with adjoint $\rho^{*} \in \mathbf{C}^{d}$ there is the inequality

$$
\operatorname{Re}\left[e^{-i \theta^{\prime}} \rho^{*} q(\xi, \eta) \rho\right] \geq \frac{1}{2}\left(1+\cos \theta^{\prime}\right) \lambda|\rho|^{2}-\frac{1}{2}\left(1-\cos \theta^{\prime}\right) \Lambda|\rho|^{2},
$$

where $\theta^{\prime}=\operatorname{Im}(\eta)$.

Proof. We note the identity

$$
\begin{aligned}
& \operatorname{Re}\left[e^{-i \theta^{\prime}} \rho^{*}\left\langle\mathbf{a}(\cdot) \partial_{\xi} \varphi(\xi, \eta, \cdot)\right\rangle\right] \\
& =-\operatorname{Re} \rho^{*}\left\langle\mathbf{a}(\cdot) e^{i \theta^{\prime}} \partial_{\bar{\xi}} \varphi\left(\xi, \eta, \tau_{0,1} \cdot\right)\right\rangle \\
& +\operatorname{Re}\left\{\left(\frac{1+e^{-i \theta^{\prime}}}{2}\right) \rho^{*}\left\langle\mathbf{a}(\cdot)\left[\partial_{\xi} \varphi(\xi, \eta, \cdot)+e^{i \theta^{\prime}} \partial_{\bar{\xi}} \varphi\left(\xi, \eta, \tau_{0,1} \cdot\right)\right]\right\rangle\right\} \\
& -\operatorname{Re}\left\{\left(\frac{1-e^{-i \theta^{\prime}}}{2}\right) \rho^{*}\left\langle\mathbf{a}(\cdot)\left[\partial_{\xi} \varphi(\xi, \eta, \cdot)-e^{i \theta^{\prime}} \partial_{\bar{\xi}} \varphi\left(\xi, \eta, \tau_{0,1} \cdot\right)\right]\right\rangle\right\} \text {. }
\end{aligned}
$$

Proceeding as in Lemma 2.8 and using the inequality (4.56) we conclude from the last identity that

$$
\operatorname{Re}\left[e^{-i \theta^{\prime}} \rho^{*} q(\xi, \eta) \rho\right] \geq-\Lambda|\rho|^{2} \sin ^{2}\left(\frac{\theta^{\prime}}{2}\right)
$$


We also have from (4.55) the identity

$$
\begin{aligned}
& \cos ^{2}\left(\frac{\theta^{\prime}}{2}\right)\left\langle\rho^{*} \mathbf{a}(\cdot) \rho\right\rangle-\operatorname{Re}\left\langle\rho^{*} \mathbf{a}(\cdot) e^{i \theta^{\prime}} \partial_{\bar{\xi}} \varphi\left(\xi, \eta, \tau_{0,1} \cdot\right)\right\rangle \\
+ & \operatorname{Re}\left\{\left(\frac{1+e^{-i \theta^{\prime}}}{2}\right)\left\langle\rho^{*} \mathbf{a}(\cdot)\left[\partial_{\xi} \varphi(\xi, \eta, \cdot)+e^{i \theta^{\prime}} \partial_{\bar{\xi}} \varphi\left(\xi, \eta, \tau_{0,1} \cdot\right)\right]\right\rangle\right\} \\
= & e^{\operatorname{Re}(\eta)\left\langle|\varphi(\xi, \eta, \cdot)|^{2}\right\rangle} \\
- & \operatorname{Re} e^{-i \theta^{\prime}}\left\langle\overline{\varphi\left(\xi, \eta, \tau_{0,1} \cdot\right)}\left[I-\frac{\Lambda}{2} \partial_{\xi}^{*} \partial_{\xi}\right] \varphi(\xi, \eta, \cdot)\right\rangle \\
- & \frac{\Lambda}{4}\left[\left\langle\left|\partial_{\xi} \varphi(\xi, \eta, \cdot)\right|^{2}\right\rangle+\left\langle\left|\partial_{\bar{\xi}} \varphi(\xi, \eta, \cdot)\right|^{2}\right\rangle\right]+B^{2} \\
+ & \left\langle\left[\overline{\cos \left(\frac{\theta^{\prime}}{2}\right) e^{i \theta^{\prime} / 2} \rho+\frac{1}{2} \partial_{\xi} \varphi(\xi, \eta, \cdot)+\frac{1}{2} e^{i \theta^{\prime}} \partial_{\bar{\xi}} \varphi\left(\xi, \eta, \tau_{0,1} \cdot\right)}\right]\right. \\
& \left.\mathbf{a}(\cdot)\left[\cos \left(\frac{\theta^{\prime}}{2}\right) e^{i \theta^{\prime} / 2} \rho+\frac{1}{2} \partial_{\xi} \varphi(\xi, \eta, \cdot)+\frac{1}{2} e^{i \theta^{\prime}} \partial_{\bar{\xi}} \varphi\left(\xi, \eta, \tau_{0,1} \cdot\right)\right]\right\rangle .
\end{aligned}
$$

The inequality (4.57) follows from the last identity by arguing as in Lemma 2.8 and using the inequality (4.46) with $\Lambda$ replaced by $\Lambda / 2$.

Lemma 4.8. Suppose $2 d \Lambda \leq 1, \mathbf{e}_{k} \cdot \xi \in \mathbf{R}, k>1, \mathbf{e}_{1} \cdot \xi, \eta \in \mathbf{C}$ satisfying (4.50). Then for any column vectors $\rho, \sigma \in \mathbf{C}^{d}$ with adjoints $\rho^{*}, \sigma^{*} \in \mathbf{C}^{d}$ there is the inequality

$$
\begin{aligned}
& \operatorname{Re}\left[e^{-i \theta^{\prime}} \sigma^{*} q(\xi, \eta) \rho\right] \geq \frac{1}{4}\left(1+\cos \theta^{\prime}\right)\left(1-\frac{1}{2 \beta}\right) \lambda\left[|\rho|^{2}+|\sigma|^{2}\right] \\
& -\frac{1}{4}\left(1-\cos \theta^{\prime}\right)\left(1+\frac{1}{2 \alpha}\right) \Lambda\left[|\rho|^{2}+|\sigma|^{2}\right]-\frac{1}{2}(\alpha+\beta) \Lambda|\rho-\sigma|^{2},
\end{aligned}
$$

where $\theta^{\prime}=\operatorname{Im}(\eta)$, and $\alpha>0, \beta>1 / 2$ are arbitrary.

Proof. We write

$$
\operatorname{Re}\left[e^{-i \theta^{\prime}} \sigma^{*} q(\xi, \eta) \rho\right]=\operatorname{Re}\left[e^{-i \theta^{\prime}} \rho^{*} q(\xi, \eta) \rho\right]+\operatorname{Re}\left[e^{-i \theta^{\prime}}(\sigma-\rho)^{*} q(\xi, \eta) \rho\right] .
$$

We have already observed in Lemma 4.7 that

$$
\begin{aligned}
& \operatorname{Re}\left[e^{-i \theta^{\prime}} \rho^{*} q(\xi, \eta) \rho\right] \geq B^{2}+D^{2}-\sin ^{2}\left(\frac{\theta^{\prime}}{2}\right)\left\langle\rho^{*} \mathbf{a}(\cdot) \rho\right\rangle \\
& -\operatorname{Re}\left\{\left(\frac{1-e^{-i \theta^{\prime}}}{2}\right) \rho^{*}\left\langle\mathbf{a}(\cdot)\left[\partial_{\xi} \varphi(\xi, \eta, \cdot)-e^{i \theta^{\prime}} \partial_{\bar{\xi}} \varphi\left(\xi, \eta, \tau_{0,1} \cdot\right)\right]\right\rangle\right\},
\end{aligned}
$$

where $D^{2}$ is the final term on the RHS of equation (4.58). We also have that

$$
\begin{gathered}
e^{-i \theta^{\prime}}(\sigma-\rho)^{*} q(\xi, \eta) \rho=\left(\frac{e^{-i \theta^{\prime}}-1}{2}\right)(\sigma-\rho)^{*}\langle\mathbf{a}(\cdot)\rangle \rho \\
+\quad(\sigma-\rho)^{*}\left\langle\mathbf{a}(\cdot)\left[\left(\frac{e^{-i \theta^{\prime}}+1}{2}\right) \rho+\frac{1}{2} e^{-i \theta^{\prime}} \partial_{\xi} \varphi(\xi, \eta, \cdot)+\frac{1}{2} \partial_{\bar{\xi}} \varphi\left(\xi, \eta, \tau_{0,1} \cdot\right)\right]\right\rangle \\
+\quad(\sigma-\rho)^{*}\left\langle\mathbf{a}(\cdot)\left[\frac{1}{2} e^{-i \theta^{\prime}} \partial_{\xi} \varphi(\xi, \eta, \cdot)-\frac{1}{2} \partial_{\bar{\xi}} \varphi\left(\xi, \eta, \tau_{0,1} \cdot\right)\right]\right\rangle .
\end{gathered}
$$


We apply the Schwarz inequality to the last equation. Thus for arbitrary positive numbers $\alpha, \beta, \gamma$ there is the inequality

$$
\begin{gathered}
\left|e^{-i \theta^{\prime}}(\sigma-\rho)^{*} q(\xi, \eta) \rho\right| \leq \frac{\alpha}{2}(\sigma-\rho)^{*}\langle\mathbf{a}(\cdot)\rangle(\sigma-\rho) \\
+\frac{1}{2 \alpha} \sin ^{2}\left(\frac{\theta^{\prime}}{2}\right) \rho^{*}\langle\mathbf{a}(\cdot)\rangle \rho+\frac{\beta}{2}(\sigma-\rho)^{*}\langle\mathbf{a}(\cdot)\rangle(\sigma-\rho) \\
+\frac{1}{2 \beta} D^{2}+\frac{\gamma}{2}(\sigma-\rho)^{*}\left\langle\Lambda I_{d}-\mathbf{a}(\cdot)\right\rangle(\sigma-\rho)+\frac{1}{2 \gamma} B^{2} .
\end{gathered}
$$

If we apply the Schwarz inequality to the last term on the RHS of (4.61) we see that for any $\delta>0$ there is the inequality

$$
\begin{aligned}
R e\left[e^{-i \theta^{\prime}} \rho^{*} q(\xi, \eta) \rho\right] & \geq B^{2}\left[1-\frac{1}{\delta}\right]+D^{2} \\
-\sin ^{2}\left(\frac{\theta^{\prime}}{2}\right)\left\langle\rho^{*} \mathbf{a}(\cdot) \rho\right\rangle & -\delta \sin ^{2}\left(\frac{\theta^{\prime}}{2}\right) \rho^{*}\left\langle\Lambda I_{d}-\mathbf{a}(\cdot)\right\rangle \rho .
\end{aligned}
$$

We choose now $\delta=1+1 / 2 \alpha, \gamma=\beta+\alpha$. Hence the last two inequalities and (4.60) yield

$$
\begin{aligned}
\operatorname{Re}\left[e^{-i \theta^{\prime}} \sigma^{*} q(\xi, \eta) \rho\right] & \geq D^{2}\left[1-\frac{1}{2 \beta}\right] \\
-\left(1+\frac{1}{2 \alpha}\right) \sin ^{2}\left(\frac{\theta^{\prime}}{2}\right) \Lambda|\rho|^{2} & -\frac{1}{2}(\alpha+\beta) \Lambda|\rho-\sigma|^{2} .
\end{aligned}
$$

Using the fact that $D^{2} \geq \cos ^{2}\left(\frac{\theta^{\prime}}{2}\right) \lambda|\rho|^{2}$, we conclude that

$$
\begin{aligned}
\operatorname{Re}\left[e^{-i \theta^{\prime}} \sigma^{*} q(\xi, \eta) \rho\right] & \geq \frac{1}{2}\left(1+\cos \theta^{\prime}\right)\left(1-\frac{1}{2 \beta}\right) \lambda|\rho|^{2} \\
& -\frac{1}{2}\left(1-\cos \theta^{\prime}\right)\left(1+\frac{1}{2 \alpha}\right) \Lambda|\rho|^{2}-\frac{1}{2}(\alpha+\beta) \Lambda|\rho-\sigma|^{2} .
\end{aligned}
$$

To complete the proof of (4.59) we note

$$
\operatorname{Re}\left[e^{-i \theta^{\prime}} \sigma^{*} q(\xi, \eta) \rho\right]=\operatorname{Re}\left[e^{i \theta^{\prime}} \rho^{*} q^{*}(\xi, \eta) \sigma\right] .
$$

We argue now as in Lemma 2.6. Thus

$$
q^{*}(\xi, \eta)=\langle\mathbf{a}(\cdot)\rangle+\left\langle\Phi^{*}(\xi, \eta, \cdot) \partial_{\bar{\xi}}^{*} \mathbf{a}(\cdot)\right\rangle
$$

where $\Phi(\xi, \eta, \omega)$ is the solution to (2.11). Let $\Phi^{\prime}(\xi, \eta, \omega)$ be the solution to the equation

$$
e^{\eta} \Phi^{\prime}\left(\xi, \eta, \tau_{0,-1} \omega\right)-\Phi^{\prime}(\xi, \eta, \omega)+P \partial_{\xi}^{*} \mathbf{a}(\omega) \partial_{\xi} \Phi^{\prime}(\xi, \eta, \omega)+P \partial_{\xi}^{*} \mathbf{a}(\omega)=0
$$


Then we see that

$$
\begin{aligned}
& \left\langle\Phi^{*}(\xi, \eta, \cdot) \partial_{\bar{\xi}}^{*} \mathbf{a}(\cdot)\right\rangle=\left\langle\Phi^{*}(\xi, \eta, \cdot) P \partial_{\bar{\xi}}^{*} \mathbf{a}(\cdot)\right\rangle \\
=\quad & -\left\langle\Phi ^ { * } ( \xi , \eta , \cdot ) \left\{ e^{\bar{\eta}} \Phi^{\prime}\left(\bar{\xi}, \bar{\eta}, \tau_{0,-1} \cdot\right)-\Phi^{\prime}(\bar{\xi}, \bar{\eta}, \cdot)\right.\right. \\
+ & \left.\left.P \partial_{\bar{\xi}}^{*} \mathbf{a}(\cdot) \partial_{\bar{\xi}} \Phi^{\prime}(\bar{\xi}, \bar{\eta}, \cdot)\right\}\right\rangle \\
=\quad & -\left\langle\left\{ e^{\eta} \Phi\left(\xi, \eta, \tau_{0,1} \cdot\right)-\Phi(\xi, \eta, \cdot)\right.\right. \\
+ & \left.\left.P \partial_{\xi}^{*} \mathbf{a}(\cdot) \partial_{\xi} \Phi(\xi, \eta, \cdot)\right\}^{*} \Phi^{\prime}(\bar{\xi}, \bar{\eta}, \cdot)\right\rangle \\
=\quad & \left\langle\left\{\partial_{\xi}^{*} \mathbf{a}(\cdot)\right\}^{*} \Phi^{\prime}(\bar{\xi}, \bar{\eta}, \cdot)\right\rangle=\left\langle\mathbf{a}(\cdot) \partial_{\bar{\xi}} \Phi^{\prime}(\bar{\xi}, \bar{\eta}, \cdot)\right\rangle \cdot
\end{aligned}
$$

We conclude that

$$
q^{*}(\xi, \eta)=\langle\mathbf{a}(\cdot)\rangle+\left\langle\mathbf{a}(\cdot) \partial_{\bar{\xi}} \Phi^{\prime}(\bar{\xi}, \bar{\eta}, \cdot)\right\rangle .
$$

Using the previous formula and the argument to obtain 4.62) we conclude that

$$
\begin{gathered}
R e\left[e^{i \theta^{\prime}} \rho^{*} q^{*}(\xi, \eta) \sigma\right] \geq \frac{1}{2}\left(1+\cos \theta^{\prime}\right)\left(1-\frac{1}{2 \beta}\right) \lambda|\sigma|^{2} \\
-\frac{1}{2}\left(1-\cos \theta^{\prime}\right)\left(1+\frac{1}{2 \alpha}\right) \Lambda|\sigma|^{2}-\frac{1}{2}(\alpha+\beta) \Lambda|\sigma-\rho|^{2} .
\end{gathered}
$$

The inequality (4.59) follows now from this last inequality and (4.62).

Corollary 4.1. Suppose $2 d \Lambda \leq 1, \mathbf{e}_{k} \cdot \xi \in \mathbf{R}, k>1, \mathbf{e}_{1} \cdot \xi, \eta \in \mathbf{C}$ satisfying (4.45). Then there is the inequality

$$
\begin{aligned}
\left|e^{\eta}-1+e(\xi) q(\xi, \eta) e(-\xi)\right| \geq \frac{\left(1+\cos \theta^{\prime}\right) \lambda}{2(\cosh \theta+3)}\left[|e(\xi)|^{2}+|e(-\xi)|^{2}\right] \\
+\frac{1}{2}\left(1-\cos \theta^{\prime}\right)\left\{2-\frac{\cosh \theta+2}{\cosh \theta+3} \Lambda\left[|e(\xi)|^{2}+|e(-\xi)|^{2}\right]\right\},
\end{aligned}
$$

where $\theta^{\prime}=\operatorname{Im}(\eta), \quad \theta=\operatorname{Im}\left(\mathbf{e}_{1} \cdot \xi\right)$.

Proof. We have

$$
\left|e^{\eta}-1+e(\xi) q(\xi, \eta) e(-\xi)\right| \geq e^{\operatorname{Re}(\eta)}-\cos \theta^{\prime}+\operatorname{Re}\left[e^{-i \theta^{\prime}} e(\xi) q(\xi, \eta) e(-\xi)\right] .
$$

Now we apply Lemma 4.8 with $\alpha=\beta=[\cosh \theta+3] / 2[\cosh \theta+1]$.

Lemma 4.9. Suppose $4 d \Lambda \leq 1, \mathbf{e}_{k} \cdot \xi \in \mathbf{R}, k>1, \mathbf{e}_{1} \cdot \xi, \eta \in \mathbf{C}$ satisfy (4.45). For fixed $\operatorname{Re}(\eta), \operatorname{Im}\left(\mathbf{e}_{1} \cdot \xi\right)$ consider $q(\xi, \eta)$ as a function of $\zeta=(\operatorname{Re}(\xi), \operatorname{Im}(\eta)) \in$ $[-\pi, \pi]^{d+1}$. Then $q$ satisfies the conclusions of Lemmas 3.5 and 3.6.

Proof. Consider the function $F$ of (3.16), defined for $\xi \in \mathbf{R}^{d}, \eta \in \mathbf{C}, \operatorname{Re}(\eta)>0$. We need to show that $F$ analytically continues to the region 4.45) and that $F$, regarded as a function of $\zeta$ is in $L^{\infty}\left([-\pi, \pi]^{d+1}, \mathcal{M}\right)$ with $\|F\|_{\infty} \leq 1$. To see this it will be sufficient to show that

$$
\left|e^{\eta}-1+\Lambda e(\xi)^{T} e(-\xi)\right| \geq \Lambda|e(\xi)||e(-\xi)|,
$$


provided $(\xi, \eta)$ satisfy (4.45). To see this observe that

$$
\begin{aligned}
\Lambda|e(\xi)||e(-\xi)| & \leq \Lambda \sum_{k=2}^{d}\left|e_{k}(\xi)\right|^{2} \\
& +\frac{\Lambda}{2}\left[\left|e^{i \mathbf{e}_{1} \cdot \xi}-1\right|^{2}+\left|e^{-i \mathbf{e}_{1} \cdot \xi}-1\right|^{2}\right] \\
& =\Lambda \sum_{k=2}^{d}\left|e_{k}(\xi)\right|^{2}+\Lambda[1+\cosh 2 \theta-2 \cosh \theta \cos \beta],
\end{aligned}
$$

where $\mathbf{e}_{1} \cdot \xi=\beta+i \theta$. Using the fact that $4 d \Lambda \leq 1$ we also have that

$$
\begin{aligned}
\left|1-\Lambda e(\xi)^{T} e(-\xi)\right| & \leq 1-\Lambda \sum_{k=2}^{d}\left|e_{k}(\xi)\right|^{2}-4 \Lambda \\
& +\Lambda\left|e^{i \mathbf{e}_{1} \cdot \xi}+1\right|\left|e^{-i \mathbf{e}_{1} \cdot \xi}+1\right| \\
& =1-\Lambda \sum_{k=2}^{d}\left|e_{k}(\xi)\right|^{2}-4 \Lambda+2 \Lambda[\cosh \theta+\cos \beta]
\end{aligned}
$$

We conclude from the last two inequalities that

$$
\Lambda|e(\xi)||e(-\xi)|+\left|1-\Lambda e(\xi)^{T} e(-\xi)\right| \leq 1+2 \Lambda[\cosh \theta+3][\cosh \theta-1] .
$$

The inequality (4.63) follows from (4.66). Observe that the fact that $\|F\|_{\infty} \leq 1$ in the region (4.45) gives an alternative proof of the result that the operator $T_{\Lambda, \eta, \xi}$ on $\mathcal{H}(\Omega)$ satisfies $\left\|T_{\Lambda, \eta, \xi}\right\| \leq 1$ provided $(\xi, \eta)$ lies in the region (4.45).

To complete the proof of the lemma we need to prove analogues of the inequalities (3.12), (3.30), (3.31), for the region (4.45). The analogue of (3.12) is the inequality

$$
\left|e^{\eta}-1+\Lambda e(\xi)^{T} e(-\xi)\right| \geq\left[\frac{e^{R e(\eta)}+1}{2}\right] \Lambda|e(\operatorname{Re} \xi)|^{2},
$$

which follows from (4.65). To obtain the analogue of (3.30), observe from (4.65) that

$$
\left|e^{\eta}-1+\Lambda e(\xi)^{T} e(-\xi)\right| \geq e^{\operatorname{Re}(\eta)}-1+2 \Lambda[1-\cos \beta]-2 \Lambda[\cosh \theta-1] .
$$

We also have that

$$
\begin{aligned}
\left|e^{\eta}-1+\Lambda e(\xi)^{T} e(-\xi)\right| \geq & e^{R e(\eta)}|\sin (\operatorname{Im} \eta)|-2 \Lambda|\sin \beta||\sinh \theta| \\
& \geq e^{R e(\eta)}|\sin (\operatorname{Im} \eta)|-2 \Lambda[1-\cos \beta]-\Lambda\left[\cosh ^{2} \theta-1\right] .
\end{aligned}
$$

It follows then from the last two inequalities that

$$
\begin{aligned}
2\left|e^{\eta}-1+\Lambda e(\xi)^{T} e(-\xi)\right| \geq & e^{\operatorname{Re}(\eta)}-1+e^{\operatorname{Re}(\eta)}|\sin (\operatorname{Im} \eta)| \\
& -\Lambda[\cosh \theta+3][\cosh \theta-1] .
\end{aligned}
$$

Hence from (4.45) we have that

$$
\left|e^{\eta}-1+\Lambda e(\xi)^{T} e(-\xi)\right| \geq \frac{1}{\pi} e^{R e(\eta)}|\operatorname{Im}(\eta)|, \eta \in \mathbf{C},|\operatorname{Im}(\eta)| \leq \frac{\pi}{2} .
$$

Evidently (4.69) is the analogue of (3.30). To establish the analogue of (3.31) observe that

$$
\left|e^{\eta}-1+\Lambda e(\xi)^{T} e(-\xi)\right| \geq e^{\operatorname{Re}(\eta)}|\cos (\operatorname{Im}(\eta))|-2 \Lambda[\cosh \theta-1], \frac{\pi}{2} \leq|\operatorname{Im}(\eta)| \leq \pi .
$$


Hence from this inequality and (4.68) we have that

$$
\begin{aligned}
2\left|e^{\eta}-1+\Lambda e(\xi)^{T} e(-\xi)\right| \geq e^{R e(\eta)} \mid & \cos (\operatorname{Im}(\eta)) \mid \\
& +\frac{1}{2} e^{\operatorname{Re}(\eta)}|\sin (\operatorname{Im}(\eta))|, \quad \frac{\pi}{2} \leq|\operatorname{Im}(\eta)| \leq \pi .
\end{aligned}
$$

We conclude that

$$
\left|e^{\eta}-1+\Lambda e(\xi)^{T} e(-\xi)\right| \geq \frac{1}{2} e^{R e(\eta)}, \frac{\pi}{2} \leq|\operatorname{Im}(\eta)| \leq \pi,
$$

which is the analogue of (3.31). The proof now proceeds exactly as in Lemmas 3.5, 3.6 using (4.67), (4.69), (4.70) in place of (3.12), (3.30), (3.31).

Proof of Theorem 1.1. We are assuming $4 d \Lambda \leq 1, \mathbf{e}_{1} \cdot \xi \in \mathbf{C}$ with $\theta=\operatorname{Im}\left(\mathbf{e}_{1} \cdot \xi\right)$ and

$$
e^{R e(\eta)}-1 \rightarrow 2 \Lambda[\cosh \theta+3][\cosh \theta-1],
$$

whence we are at the limit of the inequality (4.45). Observe the inequality

$$
\begin{aligned}
|e(\operatorname{Re}(\xi))|^{2}+2 \cosh \theta[\cosh \theta-1] & \leq \frac{|e(\xi)|^{2}+|e(-\xi)|^{2}}{2} \\
& \leq|e(\operatorname{Re}(\xi))|^{2}+2[\cosh \theta+3][\cosh \theta-1] .
\end{aligned}
$$

It follows, in particular, that

$$
e^{\operatorname{Re}(\eta)}-1 \leq 2 \Lambda\left[|e(\xi)|^{2}+|e(-\xi)|^{2}\right] .
$$

We also see from Corollary 4.1 that there are universal constants $c_{0}, \theta_{0}>0$ such that

$$
\left|e^{\eta}-1+e(\xi) q(\xi, \eta) e(-\xi)\right| \geq c_{0} \lambda\left[|e(\xi)|^{2}+|e(-\xi)|^{2}\right],
$$

provided $|\theta| \leq \theta_{0}$. In view of Lemma 4.6 we also have that

$$
\left|e^{\eta}-1+e(\xi) q(\xi, \eta) e(-\xi)\right| \geq|\operatorname{Im}(\eta)| / \pi,
$$

provided $|\operatorname{Im}(\eta)| \leq \pi / 2$ and

$$
\pi \Lambda\left[|e(\xi)|^{2}+|e(-\xi)|^{2}\right] \leq|\operatorname{Im}(\eta)| .
$$

We conclude from (4.73), (4.74) that

$$
\left|e^{\eta}-1+e(\xi) q(\xi, \eta) e(-\xi)\right| \geq \frac{c_{1} \lambda}{\Lambda}\left\{|\operatorname{Im}(\eta)|+\Lambda\left[|e(\xi)|^{2}+|e(-\xi)|^{2}\right]\right\},
$$

for some universal constant $c_{1}>0$, provided $|\operatorname{Im}(\eta)| \leq \pi / 2,|\theta| \leq \theta_{0}$. It is evident also from Corollary 4.1 that there is a universal constant $c_{2}>0$ such that

$$
\left|e^{\eta}-1+e(\xi) q(\xi, \eta) e(-\xi)\right| \geq c_{2}, \frac{\pi}{2}<|\operatorname{Im}(\eta)| \leq \pi,|\theta| \leq \theta_{0} .
$$

It is evident now from (4.72), 4.75), (4.76) that the results of Lemmas 2.9, 2.10, 2.11 extend to complex $\mathbf{e}_{1} \cdot \xi$ provided $|\theta| \leq \theta_{0}$ and $\eta$ satisfies (4.71). Note that the result of Lemma 2.11 now becomes

$$
\left|\hat{G}_{\mathbf{a}}(\xi, t+1)-\hat{G}_{\mathbf{a}}(\xi, t)\right| \leq \frac{C_{\delta} \Lambda}{\lambda}\left\{\frac{\Lambda\left[|e(\xi)|^{2}+|e(-\xi)|^{2}\right]}{2}\right\}^{\delta} .
$$


We can also see that the results of Lemmas 4.1, 4.2, 4.3, 4.4 extend to complex $\mathbf{e}_{1} \cdot \xi$ provided $|\theta| \leq \theta_{0}$ and $\eta$ satisfies (4.71). The theorem follows now from Lemmas 4.1-4.4 and the observation that, for some universal constant $C$,

$$
\begin{aligned}
\min _{|\theta| \leq \theta_{0}}\{2 \Lambda[\cosh \theta+3][\cosh \theta-1] t & \left.+\theta x \cdot \mathbf{e}_{1}\right\} \\
& =-\min \left\{\left|x \cdot \mathbf{e}_{1}\right|, \quad\left|x \cdot \mathbf{e}_{1}\right|^{2} /(1+\Lambda t)\right\} / C .
\end{aligned}
$$

\section{Proof of Theorem 1.2}

We first obtain a generalization of (1.7) for the expectation of the Green's function for (1.8).

Lemma 5.1. Let $G_{\mathbf{a}}(x, t)$ be given by (1.6) where $G_{\mathbf{a}}(x, y, t, \omega)$ is the Green's function for (1.8). Then for any $\xi \in \mathbf{R}^{d}$ there is the inequality

$$
\sum_{x \in \mathbf{Z}^{d}} e^{2 x \cdot \xi} G_{\mathbf{a}}(x, t)^{2} \leq \exp \left\{2 \Lambda t \sum_{k=1}^{d}\left[\left|e^{\mathbf{e}_{k} \cdot \xi}-1\right|^{2}+\left|e^{-\mathbf{e}_{k} \cdot \xi}-1\right|^{2}\right]\right\} .
$$

Proof. We use the standard method (see Appendix A of [11]). Thus if $u(x, t, \omega)$ satisfies (1.8) with initial condition $u(x, 0, \omega)=\delta(x)$, then $G_{\mathbf{a}}(x, t)=\langle u(x, t, \cdot)\rangle$. We put $v(x, t, \omega)=e^{x \cdot \xi} u(x, t, \omega)$. Hence $v$ satisfies the equation

$$
\frac{\partial v}{\partial t}(x, t, \omega)=-e^{x \cdot \xi} \nabla^{*} \mathbf{a}\left(\tau_{x, t} \omega\right) \nabla\left[e^{-x \cdot \xi} v(x, t, \omega)\right],
$$

with initial condition $v(x, 0, \omega)=\delta(x)$. On multiplying (5.1) by $v(x, t, \omega)$ and summing over $x \in \mathbf{Z}^{d}$ we have that

$$
\frac{d}{d t} \frac{1}{2} \sum_{x \in \mathbf{Z}^{d}} v(x, t, \omega)^{2}=-\sum_{x \in \mathbf{Z}^{d}} \nabla\left[e^{x \cdot \xi} v(x, t, \omega)\right] \mathbf{a}\left(\tau_{x, t} \omega\right) \nabla\left[e^{-x \cdot \xi} v(x, t, \omega)\right] .
$$

Letting $\Phi(x, t, \omega), \Psi(x, t, \omega) \in \mathbf{R}^{d}$ be the vectors

$$
\begin{aligned}
& \Phi(x, t, \omega)=\left[\left(e^{\mathbf{e}_{1} \cdot \xi}-1\right) v\left(x+\mathbf{e}_{1}, t, \omega\right), \cdots,\left(e^{\mathbf{e}_{d} \cdot \xi}-1\right) v\left(x+\mathbf{e}_{d}, t, \omega\right)\right], \\
& \Psi(x, t, \omega)=\left[\left(e^{-\mathbf{e}_{1} \cdot \xi}-1\right) v\left(x+\mathbf{e}_{1}, t, \omega\right), \cdots,\left(e^{-\mathbf{e}_{d} \cdot \xi}-1\right) v\left(x+\mathbf{e}_{d}, t, \omega\right)\right],
\end{aligned}
$$

we see that

$$
\begin{aligned}
\frac{d}{d t} \frac{1}{2} \sum_{x \in \mathbf{Z}^{d}} v(x, t, \omega)^{2}= & -\sum_{x \in \mathbf{Z}^{d}} \nabla v(x, t, \omega) \mathbf{a}\left(\tau_{x, t} \omega\right) \nabla v(x, t, \omega) \\
& -\sum_{x \in \mathbf{Z}^{d}} \Phi(x, t, \omega) \mathbf{a}\left(\tau_{x, t} \omega\right) \nabla v(x, t, \omega) \\
& -\sum_{x \in \mathbf{Z}^{d}} \nabla v(x, t, \omega) \mathbf{a}\left(\tau_{x, t} \omega\right) \Psi(x, t, \omega) \\
& -\sum_{x \in \mathbf{Z}^{d}} \Phi(x, t, \omega) \mathbf{a}\left(\tau_{x, t} \omega\right) \Psi(x, t, \omega) .
\end{aligned}
$$

Using the Schwarz inequality in the last identity and (1.1) we conclude that

$$
\frac{d}{d t} \frac{1}{2} \sum_{x \in \mathbf{Z}^{d}} v(x, t, \omega)^{2} \leq \Lambda \sum_{x \in \mathbf{Z}^{d}} v(x, t, \omega)^{2} \sum_{k=1}^{d}\left[\left|e^{\mathbf{e}_{k} \cdot \xi}-1\right|^{2}+\left|e^{-\mathbf{e}_{k} \cdot \xi}-1\right|^{2}\right] .
$$

The result follows from this last inequality. 
Lemma 5.1 enables us to define the Fourier-Laplace transform analogously to (2.1) by

$$
\hat{G}_{\mathbf{a}}(\xi, \eta)=\int_{0}^{\infty} d t \sum_{x \in \mathbf{Z}^{d}} G_{\mathbf{a}}(x, t) \exp [i x \cdot \xi-\eta t] .
$$

In view of Lemma 5.1 the RHS of (5.2) converges absolutely for $\operatorname{Re}(\eta)>0, \xi \in$ $[-\pi, \pi]^{d}$ and $\hat{G}_{\mathbf{a}}(\xi, \eta)$ is a $C^{\infty}$ function of $(\xi, \eta)$, analytic in $\eta$. It also follows from Lemma 5.1 that $G_{\mathbf{a}}(x, t)$ can be recovered from the Fourier-Laplace transform by the inverse transform

$$
G_{\mathbf{a}}(x, t)=\lim _{N \rightarrow \infty} \frac{1}{(2 \pi)^{d+1}} \int_{[-\pi, \pi]^{d}} d \xi e^{-i x \cdot \xi} \int_{-N}^{N} d[\operatorname{Im}(\eta)] e^{\eta t} \hat{G}_{\mathbf{a}}(\xi, \eta),
$$

where $\operatorname{Re}(\eta)>0$ is kept fixed in the integration. In fact, for any $x \in \mathbf{Z}^{d}$ the RHS of (5.3) converges in measure as a function of $t$ to the LHS as $N \rightarrow \infty$.

Our first goal here will be to show that there is a $d \times d$ matrix $q(\xi, \eta)$ defined for $\xi \in[-\pi, \pi]^{d}, \operatorname{Re}(\eta)>0$ such that

$$
\hat{G}_{\mathbf{a}}(\xi, \eta)=1 /[\eta+e(\xi) q(\xi, \eta) e(-\xi)] .
$$

Proceeding as in $\S 6$ of [4] we define $q(\xi, \eta)$ by (2.24) where $\Psi(\xi, \eta, \omega)$ is the solution to (2.23). The operator $T_{\Lambda, \eta, \xi}$ in (2.23) is now the continuum limit of the operator defined by (2.30). To be specific, let $G(x, t), x \in \mathbf{Z}^{d}, t>0$, be the solution of the initial value problem

$$
\begin{aligned}
\frac{\partial G}{\partial t}(x, t)+\nabla^{*} \nabla G(x, t) & =0, \quad x \in \mathbf{Z}^{d}, t>0, \\
G(x, 0) & =\delta(x), x \in \mathbf{Z}^{d} .
\end{aligned}
$$

The Fourier transform $\hat{G}(\xi, t)$ of $G(x, t)$ is given by

$$
\hat{G}(\xi, t)=\exp \left[-|e(\xi)|^{2} t\right], \xi \in[-\pi, \pi]^{d}, t>0 .
$$

The Fourier-Laplace transform $\hat{G}(\xi, \eta)$ of $G(x, t)$ is given by

$$
\hat{G}(\xi, \eta)=1 /\left[\eta+|e(\xi)|^{2}\right] .
$$

We define the operator $T_{\Lambda, \eta, \xi}$ on $\mathcal{H}(\Omega)$ by

$$
T_{\Lambda, \eta, \xi} \varphi(\omega)=\Lambda \int_{0}^{\infty} d t e^{-\eta t} \sum_{x \in \mathbf{Z}^{d}} \nabla \nabla^{*} G(x, \Lambda t) e^{-i x \cdot \xi} \varphi\left(\tau_{x,-t} \omega\right), \omega \in \Omega .
$$

Lemma 5.2. Suppose $\operatorname{Re}(\eta)>0, \xi \in[-\pi, \pi]^{d}$. Then the operator $T_{\Lambda, \eta, \xi}$ defined by (5.6) is a bounded operator on $\mathcal{H}(\Omega)$ satisfying $\left\|T_{\Lambda, \eta, \xi}\right\| \leq 1$. The function $q(\xi, \eta)$ defined by 2.23), (2.24) is a $C^{\infty}$ function of $(\xi, \eta)$, periodic in $\xi$ and analytic in $\eta$.

Proof. To show that $T_{\Lambda, \eta, \xi}$ is bounded on $\mathcal{H}(\Omega)$ we proceed as in Lemma 3.1. Thus

$$
\left\|T_{\Lambda, \eta, \xi} \varphi\right\|^{2}=\operatorname{Tr} \int_{[-\pi, \pi]^{d} \times(-\infty, \infty)} F\left(\zeta^{\prime}-\zeta\right) d \mu_{\varphi}\left(\zeta^{\prime}\right) F\left(\zeta^{\prime}-\zeta\right)^{*},
$$


where

$$
\begin{aligned}
& \left\langle\varphi\left(\tau_{R y} \cdot\right) \varphi^{*}\left(\tau_{R y^{\prime}} \cdot\right)\right\rangle \\
& \quad=\int_{[-\pi, \pi]^{d} \times(-\infty, \infty)} e^{i\left(y-y^{\prime}\right) \cdot \zeta^{\prime}} d \mu_{\varphi}\left(\zeta^{\prime}\right), y, y^{\prime} \in \mathbf{Z}^{d} \times(-\infty, \infty),
\end{aligned}
$$

is the formula analogous to (3.6). In analogy to 3.15 , 3.16 we have that

$$
F(\xi, \operatorname{Im}(\eta))=\Lambda e(\xi) e(\xi)^{*} /\left[\bar{\eta}+\Lambda|e(\xi)|^{2}\right] .
$$

The fact that $T_{\Lambda, \eta, \xi}$ is bounded on $\mathcal{H}(\Omega)$ with $\left\|T_{\Lambda, \eta, \xi}\right\| \leq 1$ follows as in Lemma 3.1 using the fact that $\operatorname{Tr}\left\langle\varphi(\cdot) \varphi^{*}(\cdot)\right\rangle=\|\varphi\|^{2}$. The smoothness of the function $q(\xi, \eta)$ can be seen by arguing as in Lemma 2.4 .

Lemma 5.3. Suppose $\operatorname{Re}(\eta)>0, \xi \in[-\pi, \pi]^{d}$. Then for any column vector $\rho \in \mathbf{C}^{d}$ with adjoint $\rho^{*} \in \mathbf{C}^{d}$ there are the inequalities

$$
\begin{gathered}
\lambda|\rho|^{2} \leq \operatorname{Re}\left[\rho^{*} q(\xi, \eta) \rho\right] \leq \Lambda|\rho|^{2}, \\
\left|\operatorname{Im}\left[\rho^{*} q(\xi, \eta) \rho\right]\right| \leq \Lambda|\rho|^{2} .
\end{gathered}
$$

Proof. We follow the method of Lemma 6.2 of [4]. Thus, multiplying (2.23) on the right by $\rho$ and the left by $\rho^{*} \Psi(\xi, \eta, \omega)^{*}$ and taking the expectation value, we have

$$
\begin{aligned}
\rho^{*}\left\langle\Psi(\xi, \eta, \cdot)^{*} \Psi(\xi, \eta, \cdot)\right\rangle \rho=\left\langle\left[T_{\Lambda, \eta, \xi}^{*} \Psi(\xi, \eta, \cdot) \rho\right]^{*} \mathbf{b}(\cdot) \Psi(\xi, \eta, \cdot)\right\rangle \rho \\
\\
-\frac{1}{\Lambda}\left\langle\left[T_{\Lambda, \eta, \xi}^{*} \Psi(\xi, \eta, \cdot) \rho\right]^{*}\left[\mathbf{a}(\cdot)-\lambda I_{d}\right] \rho\right\rangle,
\end{aligned}
$$

where $T_{\Lambda, \eta, \xi}^{*}$ is the adjoint of $T_{\Lambda, \eta, \xi}$ on $\mathcal{H}(\Omega)$.

We can apply the Schwarz inequality to the RHS of the last equation, using the fact that the matrices $\mathbf{b}(\omega)$ and $\left[\mathbf{a}(\omega)-\lambda I_{d}\right]$ are symmetric positive definite. Thus we obtain the inequality

$$
\begin{aligned}
& \rho^{*}\left\langle\Psi(\xi, \eta, \cdot)^{*} \Psi(\xi, \eta, \cdot)\right\rangle \rho \\
\leq & \frac{1}{2}\left\langle\left[T_{\Lambda, \eta, \xi}^{*} \Psi(\xi, \eta, \cdot) \rho\right]^{*} \mathbf{b}(\cdot)\left[T_{\Lambda, \eta, \xi}^{*} \Psi(\xi, \eta, \cdot) \rho\right]\right\rangle \\
+ & \frac{1}{2}\left\langle\rho^{*} \Psi(\xi, \eta, \cdot)^{*} \mathbf{b}(\cdot) \Psi(\xi, \eta, \cdot) \rho\right\rangle \\
+ & \frac{1}{2 \Lambda}\left\langle\left[T_{\Lambda, \eta, \xi}^{*} \Psi(\xi, \eta, \cdot) \rho\right]^{*}\left[\mathbf{a}(\cdot)-\lambda I_{d}\right]\left[T_{\Lambda, \eta, \xi}^{*} \Psi(\xi, \eta, \cdot) \rho\right]\right\rangle \\
+ & \frac{1}{2 \Lambda}\left\langle\rho^{*}\left[\mathbf{a}(\cdot)-\lambda I_{d}\right] \rho\right\rangle \leq \frac{1}{2}\left\langle\left[T_{\Lambda, \eta, \xi}^{*} \Psi(\xi, \eta, \cdot) \rho\right]^{*}\left[T_{\Lambda, \eta, \xi}^{*} \Psi(\xi, \eta, \cdot)\right] \rho\right\rangle \\
+ & \frac{1}{2}\left\langle\rho^{*} \Psi(\xi, \eta, \cdot)^{*} \mathbf{b}(\cdot) \Psi(\xi, \eta, \cdot) \rho\right\rangle \\
+ & \frac{1}{2 \Lambda}\left\langle\rho^{*}\left[\mathbf{a}(\cdot)-\lambda I_{d}\right] \rho\right\rangle .
\end{aligned}
$$

Since $T_{\Lambda, \eta, \xi}^{*}$ has norm less than or equal to 1 on $\mathcal{H}(\Omega)$, we conclude that

$$
\left\langle\rho^{*} \Psi(\xi, \eta, \cdot)^{*} \mathbf{a}(\cdot) \Psi(\xi, \eta, \cdot) \rho\right\rangle \leq\left\langle\rho^{*}\left[\mathbf{a}(\cdot)-\lambda I_{d}\right] \rho\right\rangle .
$$

Hence we have that

$$
\left|\rho^{*} q(\xi, \eta) \rho-\rho^{*}\langle\mathbf{a}(\cdot)\rangle \rho\right|=\left|\rho^{*}\left\langle\left[\mathbf{a}(\cdot)-\lambda I_{d}\right] \Psi(\xi, \eta, \cdot)\right\rangle \rho\right| \leq\left\langle\rho^{*}\left[\mathbf{a}(\cdot)-\lambda I_{d}\right] \rho\right\rangle .
$$


We conclude from this last inequality that the lower bound of (5.8) holds and also (5.9)

To obtain the upper bound in (5.8) we observe again from (2.23) that

$$
\begin{aligned}
& \rho^{*}\left\langle\Psi(\xi, \eta, \cdot)^{*} \Psi(\xi, \eta, \cdot)\right\rangle \rho \\
= & \left\langle\left[T_{\Lambda, \eta, \xi}^{*} \Psi(\xi, \eta, \cdot) \rho\right]^{*} \mathbf{b}(\cdot)\left[\Psi(\xi, \eta, \cdot)+I_{d}\right] \rho\right\rangle \\
\leq & \frac{1}{2}\left\langle\left[T_{\Lambda, \eta, \xi}^{*} \Psi(\xi, \eta, \cdot) \rho\right]^{*} \mathbf{b}(\cdot)\left[T_{\Lambda, \eta, \xi}^{*} \Psi(\xi, \eta, \cdot) \rho\right]\right\rangle \\
+\quad \frac{1}{2} & \rho^{*}\left\langle\left[\Psi(\xi, \eta, \cdot)^{*}+I_{d}\right] \mathbf{b}(\cdot)\left[\Psi(\xi, \eta, \cdot)+I_{d}\right]\right\rangle \rho .
\end{aligned}
$$

Using the fact that $\left\|T_{\Lambda, \eta, \xi}^{*}\right\| \leq 1$ and (2.21) we conclude that

$$
\operatorname{Re}\left[\rho^{*}\langle\mathbf{b}(\cdot) \Psi(\xi, \eta, \cdot)\rangle \rho\right]+\frac{1}{2} \rho^{*}\langle\mathbf{b}(\cdot)\rangle \rho \geq 0 .
$$

Hence,

$$
\operatorname{Re}\left[\rho^{*} q(\xi, \eta) \rho\right]=\rho^{*}\langle\mathbf{a}(\cdot)\rangle \rho-\operatorname{Re}\left[\Lambda \rho^{*}\langle\mathbf{b}(\cdot) \Psi(\xi, \eta, \cdot)\rangle \rho\right] \leq \frac{1}{2} \rho^{*}\left\langle\Lambda I_{d}+\mathbf{a}(\cdot)\right\rangle \rho,
$$

which implies the upper bound (5.8).

Remark 7. The argument of Lemma 5.3 applies to the discrete time case provided we assume $4 d \Lambda \leq 1$.

We can deduce from Lemma 5.3 the analogues of Lemmas 2.9, 2.10, 2.11.

Lemma 5.4. Suppose $\operatorname{Re}(\eta)>0, \xi \in[-\pi, \pi]^{d}$ and $\hat{G}_{\mathbf{a}}(\xi, \eta)$ is given by (‥4). Then there is a universal constant $C$ such that

$$
\int_{0}^{\infty}\left|\hat{G}_{\mathbf{a}}(\xi, \eta)+\hat{G}_{\mathbf{a}}(\xi, \bar{\eta})\right| d[\operatorname{Im}(\eta)] \leq C\left(\frac{\Lambda}{\lambda}\right) .
$$

Proof. As in Lemma 2.9 .

Lemma 5.5. Suppose $\operatorname{Re}(\eta)>0, \xi \in[-\pi, \pi]^{d}$ and $\hat{G}_{\mathbf{a}}(\xi, \eta)$ is given by (‥4) . Then for any $t>0$, the limit

$$
\lim _{N \rightarrow \infty} \int_{0}^{N} \hat{G}_{\mathbf{a}}(\xi, \eta) \sin [\operatorname{Im}(\eta) t] d[\operatorname{Im}(\eta)]
$$

exists. Further, there is a universal constant $C>0$ such that

$$
\left|\int_{0}^{\infty} \hat{G}_{\mathbf{a}}(\xi, \eta) \sin [\operatorname{Im}(\eta) t] d[\operatorname{Im}(\eta)]\right| \leq C\left(\frac{\Lambda}{\lambda}\right) .
$$

Proof. As in Lemma 2.10.

It follows from Lemmas $5.4,5.5$ that for $\hat{G}_{\mathbf{a}}(\xi, \eta)$ given by (5.4) we may define $\hat{G}_{\mathbf{a}}(\xi, t), t>0$, uniquely by

$$
\hat{G}_{\mathbf{a}}(\xi, t)=\lim _{N \rightarrow \infty} \frac{1}{2 \pi} \int_{-N}^{N} d[\operatorname{Im}(\eta)] e^{\eta t} \hat{G}_{\mathbf{a}}(\xi, \eta),
$$

where the integration in (5.11) is over any contour with fixed $\operatorname{Re}(\eta)>0$. 
Lemma 5.6. Suppose $\hat{G}_{\mathbf{a}}(\xi, t)$ is given by (5.11) for $\xi \in[-\pi, \pi]^{d}, t>0$. Then for any $\delta$ satisfying $0 \leq \delta<1$ there is a constant $C_{\delta}>0$, depending only on $\delta$ such that

$$
\left|\hat{G}_{\mathbf{a}}(\xi, t)-\hat{G}_{\mathbf{a}}\left(\xi, t^{\prime}\right)\right| \leq \frac{C_{\delta} \Lambda}{\lambda}\left[\frac{\Lambda|e(\xi)|^{2}\left|t-t^{\prime}\right|}{1+\Lambda|e(\xi)|^{2}\left|t-t^{\prime}\right|}\right]^{\delta}, t, t^{\prime}>0 .
$$

Proof. In view of Lemmas 5.4, 5.5 we have that $\left|\hat{G}_{\mathbf{a}}(\xi, t)\right| \leq C(\Lambda / \lambda)$ for some universal constant $C$. Hence we can assume that $\Lambda|e(\xi)|^{2}\left|t-t^{\prime}\right| \leq 1 / 4$. We have from (5.11) that

$$
\begin{aligned}
& \hat{G}_{\mathbf{a}}(\xi, t)-\hat{G}_{\mathbf{a}}\left(\xi, t^{\prime}\right)=\lim _{N \rightarrow \infty} \frac{1}{2 \pi} \int_{-N}^{N}\left\{e^{\eta t}-e^{\eta t^{\prime}}\right\} \hat{G}_{\mathbf{a}}(\xi, \eta) d[\operatorname{Im}(\eta)] \\
= & \lim _{N \rightarrow \infty} \frac{1}{2 \pi} \int_{-N}^{N}\left\{e^{\eta t}-e^{\eta t^{\prime}}\right\}\left[\hat{G}_{\mathbf{a}}(\xi, \eta)-\frac{1}{\eta}\right] d[\operatorname{Im}(\eta)] \\
= & \frac{1}{2 \pi} \int_{|\operatorname{Im}(\eta)|<4 \Lambda|e(\xi)|^{2}}(\cdot) d[\operatorname{Im}(\eta)]+\frac{1}{2 \pi} \int_{4 \Lambda|e(\xi)|^{2}<|\operatorname{Im}(\eta)|<\left|t-t^{\prime}\right|-1}(\cdot) d[\operatorname{Im}(\eta)] \\
+ & \lim _{N \rightarrow \infty} \frac{1}{2 \pi} \int_{\left|t-t^{\prime}\right|^{-1}<|\operatorname{Im}(\eta)|<N}(\cdot) d[\operatorname{Im}(\eta)] .
\end{aligned}
$$

Let us assume $t^{\prime}<t$. Then from Lemma 5.3 we have that

$$
\left|\int_{|\operatorname{Im}(\eta)|<4 \Lambda|e(\xi)|^{2}}(\cdot) d[\operatorname{Im}(\eta)]\right| \leq \exp [\operatorname{Re}(\eta) t] \frac{16 \Lambda}{\lambda}\left[\Lambda|e(\xi)|^{2}\left|t-t^{\prime}\right|\right]
$$

From the fundamental theorem of calculus we have that

$$
\left\{e^{\eta t}-e^{\eta t^{\prime}}\right\}\left[\hat{G}_{\mathbf{a}}(\xi, \eta)-\frac{1}{\eta}\right]=-\frac{e(\xi) q(\xi, \eta) e(-\xi)}{\eta+e(\xi) q(\xi, \eta) e(-\xi)}\left(t-t^{\prime}\right) \int_{0}^{1} d s e^{s \eta t+(1-s) \eta t^{\prime}} .
$$

Hence Lemma 5.3 implies that

$$
\begin{aligned}
& \left|\int_{4 \Lambda|e(\xi)|^{2}<|\operatorname{Im}(\eta)|<\left|t-t^{\prime}\right|^{-1}}(\cdot) d[\operatorname{Im}(\eta)]\right| \\
& \quad \leq \exp [\operatorname{Re}(\eta) t] 8 \Lambda|e(\xi)|^{2}\left|t-t^{\prime}\right| \log \left[1 / 4 \Lambda|e(\xi)|^{2}\left|t-t^{\prime}\right|\right] .
\end{aligned}
$$

Finally, we have again from Lemma 5.3 that

$$
\lim _{N \rightarrow \infty}\left|\int_{\left|t-t^{\prime}\right|-1<|\operatorname{Im}(\eta)|<N}(\cdot) d[\operatorname{Im}(\eta)]\right| \leq \exp [\operatorname{Re}(\eta) t] 8 \Lambda|e(\xi)|^{2}\left|t-t^{\prime}\right| .
$$

The result follows from the last three inequalities by letting $\operatorname{Re}(\eta) \rightarrow 0$.

It is clear that the results of Lemmas 3.5 and 3.6 apply to the function $q(\xi, \eta)$ of Lemma 5.2, the only difference being that now

$$
\frac{\partial^{m} q}{\partial \eta^{m}} \in L_{w}^{(1+d / 2) / m}\left([-\pi, \pi]^{d} \times(-\infty, \infty), \mathcal{M}\right) .
$$

Now let us define $G_{\mathbf{a}}(x, t), x \in \mathbf{Z}^{d}, t>0$, by (5.3) where $\hat{G}_{\mathbf{a}}(\xi, \eta)$ is given by (5.4). Then, in view of (5.12) the results of Lemmas 4.1, 4.2, 4.3 hold for $G_{\mathbf{a}}(x, t)$ provided we can establish the analogues of the representations (4.1), (4.20). The analogue of (4.1) is

$$
G_{\mathbf{a}}(x, t)=\frac{(-1)^{m}}{t^{m}} \lim _{N \rightarrow \infty} \int_{[-\pi, \pi]^{d}} d \xi e^{-i x \cdot \xi} \int_{-N}^{N} d[\operatorname{Im}(\eta)]\left\{e^{\eta t}-1\right\} \frac{\partial^{m} \hat{G}_{\mathbf{a}}(\xi, \eta)}{\partial \eta^{m}} .
$$


The identity (5.13) follows from (5.3), Lemma 5.3 and the next lemma.

Lemma 5.7. Let $q(\xi, \eta), \xi \in[-\pi, \pi]^{d}, \operatorname{Re}(\eta)>0$, be the function of Lemma 5.2. Then for any $\rho \in \mathbf{C}^{d}, m=1,2, \ldots$ there is the inequality

$$
\left|\rho^{*} \frac{\partial^{m} q(\xi, \eta)}{\partial \eta^{m}} \rho\right| \leq C_{m} \Lambda|\rho|^{2}\left(\frac{\Lambda}{\lambda}\right)^{m+1}[\operatorname{Re}(\eta)]^{-m},
$$

where the constant $C_{m}$ depends only on $m$.

Proof. From (2.24) we have that

$$
\frac{\partial^{m} q(\xi, \eta)}{\partial \eta^{m}}=-\Lambda\left\langle\mathbf{b}(\cdot) \frac{\partial^{m} \Psi(\xi, \eta, \cdot)}{\partial \eta^{m}}\right\rangle, \quad m \geq 1,
$$

where $\Psi(\xi, \eta, \omega)$ is given by (2.31). The result follows then if we can show that the operator $\partial^{k} T_{\Lambda, \eta, \xi} / \partial \eta^{k}, k=0,1,2, \ldots$, is a bounded operator on $\mathcal{H}(\Omega)$ with norm $\left\|\partial^{k} T_{\Lambda, \eta, \xi} / \partial \eta^{k}\right\| \leq[\operatorname{Re}(\eta)]^{-k}$. This last fact follows by the argument of Lemma 3.1 .

The analogue of Lemma 4.4 is the following:

Lemma 5.8. Let $G_{\mathbf{a}}(x, t), x \in \mathbf{Z}^{d}, t>0$, be defined by (5.3) where $\hat{G}_{\mathbf{a}}(\xi, \eta)$ is given by (5.4). Let $\gamma$ satisfy $0 \leq \gamma<1$. Then there is a constant $C_{d, \gamma}$ depending only on $d, \gamma$ such that

$$
\left|G_{\mathbf{a}}\left(x, t^{\prime}\right)-G_{\mathbf{a}}(x, t)\right| \leq \frac{\left[\Lambda\left|t^{\prime}-t\right|\right]^{\gamma} C_{d, \gamma}(\Lambda / \lambda)^{3 d+6}}{1+(\Lambda t)^{d / 2+\gamma}}
$$

provided $\left|t-t^{\prime}\right| \leq t / 2$.

Proof. If $\Lambda t \leq 1$, the result follows from Lemma 5.6. Hence we shall assume $\Lambda t>1$. The representation corresponding to (4.37) is given by

$$
\begin{aligned}
& (2 \pi)^{d+1}\left[G_{\mathbf{a}}\left(x, t^{\prime}\right)-G_{\mathbf{a}}(x, t)\right]=\frac{1}{2} \frac{(-1)^{m+1}}{t^{m}} \lim _{N \rightarrow \infty} \int_{[-\pi, \pi]^{d}} d \xi e^{-i x \cdot \xi} \\
& \int_{-N}^{N} d[\operatorname{Im}(\eta)]\left\{e^{\eta t^{\prime}}-e^{\eta t}\right\}\left\{\frac{\partial^{m} \hat{G}_{\mathbf{a}}(\xi, \eta+\pi i / t)}{\partial \eta^{m}}-\frac{\partial^{m} \hat{G}_{\mathbf{a}}(\xi, \eta)}{\partial \eta^{m}}\right\} \\
& +\frac{1}{4} \frac{(-1)^{m+1}}{t^{m}} \lim _{N \rightarrow \infty} \int_{[-\pi, \pi]^{d}} d \xi e^{-i x \cdot \xi} \int_{-N}^{N} d[\operatorname{Im}(\eta)]\left\{e^{\eta t^{\prime}}-1\right\} \\
& \left\{\exp \left[\frac{\pi i\left(t^{\prime}-t\right)}{t}\right]-1\right\}\left\{\frac{\partial^{m} \hat{G}_{\mathbf{a}}(\xi, \eta+\pi i / t)}{\partial \eta^{m}}-\frac{\partial^{m} \hat{G}_{\mathbf{a}}\left(\xi, \eta+\pi i / t-\pi i / t^{\prime}\right)}{\partial \eta^{m}}\right\} .
\end{aligned}
$$

The result follows now by following the argument of Lemma 4.4 .

Next we wish to show that one can analytically continue the function $q(\xi, \eta), \xi \in$ $\mathbf{R}^{d}, \operatorname{Re}(\eta)>0$, in $\xi$. The analogue of Lemma 4.5 is the following:

Lemma 5.9. The function $q(\xi, \eta), \xi \in \mathbf{R}^{d}, \operatorname{Re}(\eta)>0$, extends analytically to the region $\mathbf{e}_{1} \cdot \xi \in \mathbf{C}$ satisfying

$$
\operatorname{Re}(\eta)>2 \Lambda[\cosh \theta+1][\cosh \theta-1], \quad \theta=\operatorname{Im}\left(\mathbf{e}_{1} \cdot \xi\right) .
$$


Proof. It will be sufficient to show that the operator $T_{\Lambda, \eta, \xi}$ of (5.6) on $\mathcal{H}(\Omega)$ extends analytically to the region (5.14) and one continues to have $\left\|T_{\Lambda, \eta, \xi}\right\| \leq 1$. In view of the formula (5.7) it will be enough to show that if $(\xi, \eta)$ satisfy (5.14), then

$$
\left|\eta+\Lambda e(\xi)^{T} e(-\xi)\right| \geq \frac{\Lambda}{2}\left[|e(\xi)|^{2}+|e(-\xi)|^{2}\right] .
$$

We have that

$$
\left|\eta+\Lambda e(\xi)^{T} e(-\xi)\right| \geq \operatorname{Re}(\eta)+\Lambda \sum_{k=2}^{d}\left|e_{k}(\xi)\right|^{2}+2 \Lambda[1-\cos \beta \cosh \theta],
$$

where $\mathbf{e}_{1} \cdot \xi=\beta+i \theta$. The inequality (5.15) follows from (5.16) and (4.64) provided (5.14) holds. Note that one can also bound the LHS of (5.15) below by $|\operatorname{Im}(\eta)| / 2$. In fact, we have

$$
\begin{aligned}
\left|\eta+\Lambda e(\xi)^{T} e(-\xi)\right| & \geq|\operatorname{Im}(\eta)|-2 \Lambda|\sin \beta||\sinh \theta| \\
& \geq|\operatorname{Im}(\eta)|-2 \Lambda[1-\cos \beta]-\Lambda\left[\cosh ^{2} \theta-1\right] .
\end{aligned}
$$

Adding this last inequality to (5.16) we have

$$
\begin{aligned}
2\left|\eta+\Lambda e(\xi)^{T} e(-\xi)\right| & \geq \operatorname{Re}(\eta)+|\operatorname{Im}(\eta)|-\Lambda[\cosh \theta+3][\cosh \theta-1] \\
& \geq|\operatorname{Im}(\eta)|,
\end{aligned}
$$

since we assume (5.14) holds. We conclude from (5.15), (5.17) that in the region (5.14) there is the inequality

$$
\left|\eta+\Lambda e(\xi)^{T} e(-\xi)\right| \geq \frac{1}{3}\left\{|\operatorname{Im}(\eta)|+\frac{\Lambda}{2}\left[|e(\xi)|^{2}+|e(-\xi)|^{2}\right]\right\} .
$$

Remark 8. The conclusion of Lemma 4.9 holds for $\operatorname{Re}(\eta)$ satisfying (5.14) in view of (5.18). Here we are using the fact that $|e(\xi)|^{2}+|e(-\xi)|^{2} \geq 2|e(\operatorname{Re} \xi)|^{2}$.

Lemma 5.10. Suppose $\xi \in \mathbf{R}^{d}$, Re $(\eta)>0$ satisfy (5.14). Then the function $q(\xi, \eta)$ satisfies the inequalities (5.8), (5.9). Further, for $\sigma, \rho \in \mathbf{C}^{d}$ with adjoints $\sigma^{*}, \rho^{*}$ there is the inequality

$$
\operatorname{Re}\left[\sigma^{*} q(\xi, \eta) \rho\right] \geq \frac{\lambda}{2}\left[|\sigma|^{2}+|\rho|^{2}\right]-\frac{\Lambda}{2}|\sigma-\rho|^{2} .
$$

Proof. We have already observed in Lemma 5.9 that if (5.14) holds, then $\left\|T_{\Lambda, \eta, \xi}\right\| \leq$ 1. Since the only assumption on $T_{\Lambda, \eta, \xi}$ in the proof of Lemma 5.3 is that $\left\|T_{\Lambda, \eta, \xi}\right\| \leq$ 1 , it follows that (5.8), (5.9) hold in this case also. To prove (5.19) we use the inequality (5.10). Thus

$$
\begin{aligned}
& \operatorname{Re}\left[\sigma^{*}\langle\mathbf{a}(\cdot) \Psi(\xi, \eta, \cdot)\rangle \rho\right] \\
= & \operatorname{Re}\left[\sigma^{*}\left\langle\left[\mathbf{a}(\cdot)-\lambda I_{d}\right] \Psi(\xi, \eta, \cdot)\right\rangle \rho\right] \\
\geq & -\frac{1}{2} \sigma^{*}\left\langle\left[\mathbf{a}(\cdot)-\lambda I_{d}\right]\right\rangle \sigma-\frac{1}{2} \rho^{*}\left\langle\left[\mathbf{a}(\cdot)-\lambda I_{d}\right]\right\rangle \rho,
\end{aligned}
$$

on using (5.10). We also have that

$$
\begin{aligned}
\operatorname{Re}\left[\sigma^{*}\langle\mathbf{a}(\cdot)\rangle \rho\right] & =\frac{1}{2} \sigma^{*}\langle\mathbf{a}(\cdot)\rangle \sigma+\frac{1}{2} \rho^{*}\langle\mathbf{a}(\cdot)\rangle \rho \\
& -\frac{1}{2}\left(\sigma^{*}-\rho^{*}\right)\langle\mathbf{a}(\cdot)\rangle(\sigma-\rho) .
\end{aligned}
$$

Adding the last two inequalities we obtain (5.19). 
Proof of Theorem 1.2. If we assume that $\hat{G}_{\mathbf{a}}(\xi, \eta)$ is given by (5.4) we can proceed exactly as in the proof of Theorem 1.1 at the end of $\S 4$. Note that (5.19) implies that, provided (5.14) holds,

$$
R e[\eta+e(\xi) q(\xi, \eta) e(-\xi)] \geq \frac{\lambda}{2}\left[|e(\xi)|^{2}+|e(-\xi)|^{2}\right] .
$$

We are left then to establish (5.4). First we define an operator $S_{\Lambda, \eta, \xi}: \mathcal{H}(\Omega) \rightarrow$ $L^{2}(\Omega)$ by

$$
S_{\Lambda, \eta, \xi} \varphi(\omega)=\Lambda \int_{0}^{\infty} d t e^{-\eta t} \sum_{x \in \mathbf{Z}^{d}} \nabla G(x, \Lambda t) e^{-i x \cdot \xi} \varphi\left(\tau_{x,-t} \omega\right), \omega \in \Omega,
$$

where here we regard $\nabla G$ as a row vector in $\mathbf{R}^{d}$. Evidently $S_{\Lambda, \eta, \xi}$ is a bounded operator for $\xi \in[-\pi, \pi]^{d}, \operatorname{Re}(\eta)>0$. Let $\Psi(\xi, \eta, \omega)$ be the solution to (2.23), where the operator $T_{\Lambda, \eta, \xi}$ is given by (5.6). We can then define a row vector $\Phi(\xi, \eta, \omega) \in \mathbf{C}^{d}$ by

$$
\Phi(\xi, \eta, \omega)=P S_{\Lambda, \eta, \xi}\left[\mathbf{b}(\cdot)\left\{\Psi(\xi, \eta, \cdot)+I_{d}\right\}\right](\omega), \xi \in[-\pi, \pi]^{d}, \operatorname{Re}(\eta)>0, \omega \in \Omega .
$$

It is clear that $\Phi(\xi, \eta, \omega)$ corresponds to the solution of (2.11). Next we define the function $\hat{v}(\xi, \eta, \omega)$ for $\xi \in[-\pi, \pi]^{d}, \operatorname{Re}(\eta)>0, \omega \in \Omega$ by

$$
\hat{v}(\xi, \eta, \omega)=\hat{G}_{\mathbf{a}}(\xi, \eta) \hat{f}(\xi)[1-\Phi(\xi, \eta, \omega) e(-\xi)],
$$

where $\Phi$ is given by (5.21),$\hat{G}_{\mathbf{a}}(\xi, \eta)$ is given by (5.4) and $f:[-\pi, \pi]^{d} \rightarrow \mathbf{C}$ is an arbitrary function with bounded Fourier transform. Observe now that for fixed $\operatorname{Re}(\eta)>0$ if we regard the function $\hat{v}$ in (5.22) as a function of $\xi \in[-\pi, \pi]^{d}, \operatorname{Im}(\eta) \in$ $\mathbf{R}, \omega \in \Omega$, then it is easy to see that $\hat{v}$ is in $L^{2}\left([-\pi, \pi]^{d} \times \mathbf{R} \times \Omega\right)$. Hence if we define the function $v(x, t, \omega)$ by

$$
\begin{aligned}
& v(x, t, \omega)=\lim _{N \rightarrow \infty} \frac{1}{(2 \pi)^{d+1}} \int_{[-\pi, \pi]^{d}} d \xi e^{-i x \cdot \xi} \\
& \quad \int_{-N}^{N} d[\operatorname{Im}(\eta)] e^{\eta t} \hat{v}(\xi, \eta, \omega), x \in \mathbf{Z}^{d}, t>0, \omega \in \Omega,
\end{aligned}
$$

then it is clear that the function $e^{-R e(\eta) t} v(x, t, \omega)$ is in $L^{2}\left(\mathbf{Z}^{d} \times(0, \infty) \times \Omega\right)$. We may therefore define a function $u(x, t, \omega)$ by

$$
u(x, t, \omega)=v\left(x, t, \tau_{x, t} \omega\right), x \in \mathbf{Z}^{d}, t>0, \omega \in \Omega .
$$

It is clear that $u$ is a measurable function of $(x, t, \omega)$ and $e^{-R e(\eta) t} u(x, t, \omega)$ is in $L^{2}\left(\mathbf{Z}^{d} \times(0, \infty) \times \Omega\right)$. We shall show that $u(x, t, \omega)$ is a weak solution to the initial value problem (1.8) with initial condition $f(x, \omega)=f(x)$.

Lemma 5.11. Let $g: \mathbf{Z}^{d} \times[0, \infty) \rightarrow \mathbf{C}$ be a function of compact support which is $C^{\infty}$ in $t, t \geq 0$. Then if $u(x, t, \omega)$ is defined by (5.24), there is the identity

$$
\begin{aligned}
\sum_{x \in \mathbf{Z}^{d}} g(x, 0) f(x) & +\sum_{x \in \mathbf{Z}^{d}} \int_{0}^{\infty} \frac{\partial g}{\partial t}(x, t) u(x, t, \omega) d t \\
& =\sum_{x \in \mathbf{Z}^{d}} \int_{0}^{\infty} \nabla g(x, t) \mathbf{a}\left(\tau_{x, t} \omega\right) \nabla u(x, t, \omega) d t
\end{aligned}
$$

with probability 1 for $\omega \in \Omega$. 
Proof. We already defined just after (2.4) the gradient operator $\partial_{\xi}: L^{2}(\Omega) \rightarrow$ $\mathcal{H}(\Omega), \quad \xi \in[\pi, \pi]^{d}$. We can therefore combine $\partial_{\xi}$ with the operator $S_{\Lambda, \eta, \xi}$ of (5.20) to obtain an operator $\partial_{\xi} S_{\Lambda, \eta, \xi}: \mathcal{H}(\Omega) \rightarrow \mathcal{H}(\Omega)$. It is easy to see that $\partial_{\xi} S_{\Lambda, \eta, \xi}=T_{\Lambda, \eta, \xi}$, where $T_{\Lambda, \eta, \xi}$ is given by (5.6). Hence from (5.21) and (2.23) we have that $\partial_{\xi} \Phi(\xi, \eta, \omega)=\Psi(\xi, \eta, \omega)$. One can see from (5.23), (5.24) that $\nabla u(x, t, \omega)=\partial v\left(x, t, \tau_{x, t} \omega\right)$, where

$$
\begin{aligned}
\partial v(x, t, \omega)= & \lim _{N \rightarrow \infty} \frac{1}{(2 \pi)^{d+1}} \int_{[-\pi, \pi]^{d}} d \xi e^{-i x \cdot \xi} \\
& \int_{-N}^{N} d[\operatorname{Im}(\eta)] e^{\eta t} \partial_{\xi} \hat{v}(\xi, \eta, \omega) .
\end{aligned}
$$

From (5.22) we have that

$$
\partial_{\xi} \hat{v}(\xi, \eta, \omega)=-\hat{G}_{\mathbf{a}}(\xi, \eta) \hat{f}(\xi)[e(-\xi)+\Psi(\xi, \eta, \omega) e(-\xi)] .
$$

Suppose now that $h: \mathbf{Z}^{d} \times[0, \infty) \rightarrow \mathbf{C}$ is a continuous function with compact support. For $\xi \in[-\pi, \pi]^{d}, \operatorname{Re}(\eta)>0$ we can define an operator $A_{h, \eta, \xi}$ on $L^{2}(\Omega)$ by

$$
A_{h, \eta, \xi} \varphi(\omega)=\int_{0}^{\infty} d t e^{\eta t} \sum_{x \in \mathbf{Z}^{d}} h(x, t) e^{-i x \cdot \xi} \varphi\left(\tau_{x, t} \omega\right), \varphi \in L^{2}(\Omega), \omega \in \Omega
$$

Similarly, if $h: \mathbf{Z}^{d} \times[0, \infty) \rightarrow \mathbf{C}^{d}$ is continuous with compact support we can define an operator $A_{h, \eta, \xi}: \mathcal{H}(\Omega) \rightarrow L^{2}(\Omega)$ by the formula (5.28), where we now regard $h(x, t) \in \mathbf{C}^{d}$ as a row vector. One can easily verify the operator identity

$$
A_{\nabla g, \eta, \xi} T_{\Lambda, \eta, \xi}=A_{\nabla g, \eta, \xi}+\frac{1}{\Lambda} A_{\partial g / \partial t, \eta, \xi} S_{\Lambda, \eta, \xi}
$$

where $T_{\Lambda, \eta, \xi}$ is given by (5.6) and $S_{\Lambda, \eta, \xi}$ by (5.20). Here we are using the equation (5.5).

We consider the term on the RHS of (5.25). In view of (5.26), (5.28) we have that

$$
\begin{aligned}
& \sum_{x \in \mathbf{Z}^{d}} \int_{0}^{\infty} \nabla g(x, t) \mathbf{a}\left(\tau_{x, t} \omega\right) \nabla u(x, t, \omega) d t \\
& \quad=\lim _{N \rightarrow \infty} \frac{1}{(2 \pi)^{d+1}} \int_{[-\pi, \pi]^{d}} d \xi \int_{-N}^{N} d[\operatorname{Im}(\eta)] A_{\nabla g, \eta, \xi}\left[\mathbf{a}(\cdot) \partial_{\xi} \hat{v}(\xi, \eta, \cdot)\right](\omega)
\end{aligned}
$$

Observe now that

$$
\begin{aligned}
& A_{\nabla g, \eta, \xi}[\mathbf{a}(\cdot) \Psi(\xi, \eta, \cdot)](\omega)=\Lambda A_{\nabla g, \eta, \xi}[\Psi(\xi, \eta, \cdot)](\omega) \\
- & \Lambda A_{\nabla g, \eta, \xi}[\mathbf{b}(\cdot) \Psi(\xi, \eta, \cdot)](\omega) \\
=- & \Lambda A_{\nabla g, \eta, \xi}[\langle\mathbf{b}(\cdot) \Psi(\xi, \eta, \cdot)\rangle] \\
+ & \Lambda A_{\nabla g, \eta, \xi}[\Psi(\xi, \eta, \cdot)](\omega)-\Lambda A_{\nabla g, \eta, \xi}[P \mathbf{b}(\cdot) \Psi(\xi, \eta, \cdot)](\omega) .
\end{aligned}
$$


We have from (2.23), (5.29) that

$$
\begin{aligned}
& \Lambda A_{\nabla g, \eta, \xi}[\Psi(\xi, \eta, \cdot)](\omega)-\Lambda A_{\nabla g, \eta, \xi}[P \mathbf{b}(\cdot) \Psi(\xi, \eta, \cdot)](\omega) \\
= & \Lambda A_{\nabla g, \eta, \xi}[\Psi(\xi, \eta, \cdot)](\omega)-\Lambda A_{\nabla g, \eta, \xi}\left[T_{\Lambda, \eta, \xi} P \mathbf{b}(\cdot) \Psi(\xi, \eta, \cdot)\right](\omega) \\
+ & A_{\partial g / \partial t, \eta, \xi} S_{\Lambda, \eta, \xi}[P \mathbf{b}(\cdot) \Psi(\xi, \eta, \cdot)](\omega) \\
= & -A_{\nabla g, \eta, \xi}\left[T_{\Lambda, \eta, \xi} P \mathbf{a}(\cdot)\right](\omega) \\
+ & A_{\partial g / \partial t, \eta, \xi} S_{\Lambda, \eta, \xi}[P \mathbf{b}(\cdot) \Psi(\xi, \eta, \cdot)](\omega) .
\end{aligned}
$$

Letting $\hat{g}$ denote the Fourier-Laplace transform of $g$, we conclude that

$$
\begin{aligned}
A_{\nabla g, \eta, \xi}[\mathbf{a}(\cdot) \Psi(\xi, \eta, \cdot)](\omega) & =-\hat{g}(-\xi,-\eta) e(\xi)[q(\xi, \eta)-\langle\mathbf{a}(\cdot)\rangle] \\
-A_{\nabla g, \eta, \xi}\left[T_{\Lambda, \eta, \xi} P \mathbf{a}(\cdot)\right](\omega) & +A_{\partial g / \partial t, \eta, \xi} S_{\Lambda, \eta, \xi}[P \mathbf{b}(\cdot) \Psi(\xi, \eta, \cdot)](\omega) .
\end{aligned}
$$

From the last equation, (5.27), (5.21), (5.22) we see then that

$$
\begin{aligned}
& A_{\nabla g, \eta, \xi}\left[\mathbf{a}(\cdot) \partial_{\xi} \hat{v}(\xi, \eta, \cdot)\right](\omega) \\
= & \hat{G}_{\mathbf{a}}(\xi, \eta) \hat{f}(\xi)\{\hat{g}(-\xi,-\eta) e(\xi) q(\xi, \eta) e(-\xi) \\
- & A_{\nabla g, \eta, \xi}[P \mathbf{a}(\cdot)](\omega) e(-\xi)+A_{\nabla g, \eta, \xi}\left[T_{\Lambda, \eta, \xi} P \mathbf{a}(\cdot)\right](\omega) e(-\xi) \\
- & A_{\partial g / \partial t, \eta, \xi}[\Phi(\xi, \eta, \cdot)](\omega) e(-\xi) \\
+ & \left.A_{\partial g / \partial t, \eta, \xi}\left[P S_{\Lambda, \eta, \xi} \mathbf{b}(\cdot)\right](\omega) e(-\xi)\right\} .
\end{aligned}
$$

If we use (5.29) we have that

$$
A_{\nabla g, \eta, \xi}\left[T_{\Lambda, \eta, \xi} P \mathbf{a}(\cdot)\right](\omega)+A_{\partial g / \partial t, \eta, \xi}\left[P S_{\Lambda, \eta, \xi} \mathbf{b}(\cdot)\right](\omega)=A_{\nabla g, \eta, \xi}[P \mathbf{a}(\cdot)](\omega) .
$$

We conclude therefore that

$$
\begin{aligned}
& A_{\nabla g, \eta, \xi}\left[\mathbf{a}(\cdot) \partial_{\xi} \hat{v}(\xi, \eta, \cdot)\right](\omega) \\
= & \hat{G}_{\mathbf{a}}(\xi, \eta) \hat{f}(\xi) \hat{g}(-\xi,-\eta) e(\xi) q(\xi, \eta) e(-\xi) \\
+ & A_{\partial g / \partial t, \eta, \xi}[\hat{v}(\xi, \eta, \cdot)](\omega)-\hat{G}_{\mathbf{a}}(\xi, \eta) \hat{f}(\xi) A_{\partial g / \partial t, \eta, \xi}[1] .
\end{aligned}
$$

It is easy to see that

$$
A_{\partial g / \partial t, \eta, \xi}[1]=-\eta \hat{g}(-\xi,-\eta)-\tilde{g}(-\xi),
$$

where $\tilde{g}$ is the Fourier transform of the function $g(x, 0), x \in \mathbf{Z}^{d}$. Hence from the last two equations we have that

$$
\begin{aligned}
A_{\nabla g, \eta, \xi}\left[\mathbf{a}(\cdot) \partial_{\xi} \hat{v}(\xi, \eta, \cdot)\right](\omega) & =A_{\partial g / \partial t, \eta, \xi}[\hat{v}(\xi, \eta, \cdot)](\omega) \\
& +\hat{f}(\xi) \hat{g}(-\xi,-\eta)+\hat{G}_{\mathbf{a}}(\xi, \eta) \hat{f}(\xi) \tilde{g}(-\xi) .
\end{aligned}
$$

The identity (5.25) will follow from the last equation on using (15.23), (5.30) provided we can show that

$$
\lim _{N \rightarrow \infty} \frac{1}{2 \pi} \int_{-N}^{N} \hat{G}_{\mathbf{a}}(\xi, \eta) d[\operatorname{Im}(\eta)]=\frac{1}{2}, \xi \in[-\pi, \pi]^{d}, \operatorname{Re}(\eta)>0,
$$

and the identity

$$
\lim _{N \rightarrow \infty} \frac{1}{2 \pi} \int_{-N}^{N} \hat{g}(-\xi,-\eta) d[\operatorname{Im}(\eta)]=\frac{1}{2} \tilde{g}(-\xi), \xi \in[-\pi, \pi]^{d}, \operatorname{Re}(\eta)>0 .
$$


To see (5.32) observe that from Lemmas 5.2, 5.3 it follows that

$$
\lim _{N \rightarrow \infty} \frac{1}{2 \pi} \int_{-N}^{N}\left\{\hat{G}_{\mathbf{a}}(\xi, \eta)-\frac{1}{\eta}\right\} d[\operatorname{Im}(\eta)]=0, \xi \in[-\pi, \pi]^{d}, \operatorname{Re}(\eta)>0 .
$$

Now (5.32) follows from the fact that

$$
\lim _{N \rightarrow \infty} \frac{1}{2 \pi} \int_{-N}^{N} \frac{d[\operatorname{Im}(\eta)]}{\eta}=\frac{1}{2}, \operatorname{Re}(\eta)>0
$$

The identity (5.33) follows in a similar way.

It is easy to see that (5.4) follows from Lemma 5.11. In fact, from (15.23) the function $\hat{v}(\xi, \eta, \omega)$ is the Fourier-Laplace transform of $v(x, t, \omega)$ whence $\langle\hat{v}(\xi, \eta, \cdot)\rangle$ is the Fourier-Laplace transform of $\langle u(x, t, \cdot)\rangle$. Now (5.4) follows from (5.22). This completes the proof of Theorem 1.2.

\section{Proof of Theorem 1.3}

We proceed as in $\S 5$. Let $G(x, t), x \in \mathbf{R}^{d}, t>0$, be the solution of the initial value problem

$$
\begin{gathered}
\frac{\partial G}{\partial t}+\nabla^{*} \nabla G(x, t)=0, x \in \mathbf{R}^{d}, t>0, \\
G(x, 0)=\delta(x), \quad x \in \mathbf{R}^{d},
\end{gathered}
$$

where $\delta$ is now the Dirac $\delta$ function. The Fourier transform $\hat{G}(\xi, t)$ of $G(x, t)$ is given by

$$
\hat{G}(\xi, t)=\exp \left[-|\xi|^{2} t\right], \quad \xi \in \mathbf{R}^{d}, t>0 .
$$

The Fourier-Laplace transform $\hat{G}(\xi, \eta)$ of $G(x, t)$ is given by

$$
\hat{G}(\xi, \eta)=1 /\left[\eta+|\xi|^{2}\right], \quad \xi \in \mathbf{R}^{d}, \operatorname{Re}(\eta)>0 .
$$

The function $G(x, t)$ is, explicitly,

$$
G(x, t)=\frac{1}{(4 \pi t)^{d / 2}} \exp \left[-\frac{|x|^{2}}{4 t}\right], \quad x \in \mathbf{R}^{d}, t>0 .
$$

The operator $T_{\Lambda, \eta, \xi}$ on $\mathcal{H}(\Omega)$ is defined as in (5.6) but replacing summation over $\mathbf{Z}^{d}$ by integration over $\mathbf{R}^{d}$. Thus

$$
T_{\Lambda, \eta, \xi} \varphi(\omega)=\Lambda \int_{0}^{\infty} d t e^{-\eta t} \int_{\mathbf{R}^{d}} d x \nabla \nabla^{*} G(x, \Lambda t) e^{-i x \cdot \xi} \varphi\left(\tau_{x,-t} \omega\right), \omega \in \Omega .
$$

It is clear that $\left\|T_{\Lambda, \eta, \xi}\right\| \leq 1, \quad \xi \in \mathbf{R}^{d}, \operatorname{Re}(\eta)>0$, whence we can define $q(\xi, \eta)$ by (2.23), (2.24) $, \xi \in \mathbf{R}^{d}, \operatorname{Re}(\eta)>0$. Following the argument of Lemma 5.2 , it is clear that $q(\xi, \eta)$ is a $C^{\infty}$ function of $(\xi, \eta)$, analytic in $\eta$. We can also see that the argument of Lemma 5.3 applies here. Hence the inequalities (5.8), (5.9) hold. In analogy to (5.4) we define the function $\hat{G}_{\mathbf{a}}(\xi, \eta)$ by

$$
\hat{G}_{\mathbf{a}}(\xi, \eta)=1 /[\eta+\xi q(\xi, \eta) \xi], \quad \xi \in \mathbf{R}^{d}, \quad \operatorname{Re}(\eta)>0 .
$$

The results of Lemmas 3.5 and 3.6 apply to the function $q(\xi, \eta)$ defined here. Now one has

$$
\frac{\partial^{m} q}{\partial \eta^{m}} \in L_{w}^{(1+d / 2) / m}\left(\mathbf{R}^{d} \times(-\infty, \infty), \mathcal{M}\right) .
$$


For $M>0, x \in \mathbf{R}^{d}, t>0$, we define $G_{\mathbf{a}, M}(x, t)$ by

$$
G_{\mathbf{a}, M}(x, t)=\lim _{N \rightarrow \infty} \frac{1}{(2 \pi)^{d+1}} \int_{[-M, M]^{d}} d \xi e^{-i x \cdot \xi} \int_{-N}^{N} d[\operatorname{Im}(\eta)] e^{\eta t} \hat{G}_{\mathbf{a}}(\xi, \eta),
$$

where $\hat{G}_{\mathbf{a}}(\xi, \eta)$ is given by (6.1). Since Lemmas 5.4, 5.5 continue to hold in this situation, the limit of (6.2) exists. Since Lemma 5.7 holds in this context also, we have by integration by parts the analogue of (5.13),

$G_{\mathbf{a}, M}(x, t)=\frac{(-1)^{m}}{t^{m}} \lim _{N \rightarrow \infty} \int_{[-M, M]^{d}} d \xi e^{-i x \cdot \xi} \int_{-N}^{N} d[\operatorname{Im}(\eta)]\left\{e^{\eta t}-1\right\} \frac{\partial^{m} \hat{G}_{\mathbf{a}}(\xi, \eta)}{\partial \eta^{m}}$.

The analogue of Lemma 4.1 is now the following:

Lemma 6.1. Let $G_{\mathbf{a}, M}(x, t)$ be defined by (6.2), where $\hat{G}_{\mathbf{a}}(\xi, \eta)$ is given by (6.1). Suppose $d$ is odd, $x \in \mathbf{R}^{d}, t>0$. Then there is a positive constant $C_{d}$, depending only on $d$, such that

$$
\left|G_{\mathbf{a}, M}(x, t)\right| \leq \frac{C_{d}(\Lambda / \lambda)^{3 d+4}}{(\Lambda t)^{d / 2}} .
$$

Further, there is a function $G_{\mathbf{a}}(x, t), x \in \mathbf{R}^{d}, t>0$, such that

$$
\lim _{M \rightarrow \infty} G_{\mathbf{a}, M}(x, t)=G_{\mathbf{a}}(x, t), x \in \mathbf{R}^{d}, t>0 .
$$

The limit in (6.4) is uniform with respect to $x \in \mathbf{R}^{d}$.

Proof. The argument is the same as Lemma 4.1. One notes that

$$
\left|G_{\mathbf{a}}(x, t)-G_{\mathbf{a}, M}(x, t)\right| \leq \frac{C_{d, \delta}(\Lambda / \lambda)^{3 d+4}}{\left(\Lambda t M^{2}\right)^{\delta}(\Lambda t)^{d / 2}},
$$

provided $\delta>0$ is sufficiently small and $C_{d, \delta}$ is a constant depending only on $d$ and $\delta$. The uniform convergence in (6.4) follows.

The analogue of Lemma 4.2 holds here by an identical argument. We consider next the analogue of Lemma 4.3.

Lemma 6.2. The function $G_{\mathbf{a}}(x, t), x \in \mathbf{R}^{d}, t>0$ defined by (6.4) is $C^{1}$ in $x$, and there is a positive constant $C_{d}$, depending only on $d$, such that

$$
\left|\nabla G_{\mathbf{a}}(x, t)\right| \leq \frac{C_{d}(\Lambda / \lambda)^{3 d+6}}{(\Lambda t)^{d / 2+1 / 2}} .
$$

Let $\delta$ satisfy $1 / 2 \leq \delta<1$. Then there is a constant $C_{d, \delta}$ depending only on $d, \delta$ such that

$$
\left|\nabla G_{\mathbf{a}}(x, t)-\nabla G_{\mathbf{a}}\left(x^{\prime}, t\right)\right| \leq \frac{\left|x-x^{\prime}\right|^{2 \delta-1} C_{d, \delta}(\Lambda / \lambda)^{3 d+6}}{(\Lambda t)^{d / 2+\delta}} .
$$

Proof. We proceed as in Lemma 4.3. To prove (6.5) we use the inequality

$$
\left|e^{-i x \cdot \xi}-e^{-i x^{\prime} \cdot \xi}\right| \leq 2^{(1-\delta)}\left|x-x^{\prime}\right|^{2 \delta-1}|\xi|^{2 \delta-1} .
$$


The analogue of Lemma 5.8 is given by:

Lemma 6.3. Let $\gamma$ satisfy $0 \leq \gamma<1$. If $G_{\mathbf{a}}(x, t)$ is the function defined by (6.4) there is a constant $C_{d, \gamma}$ depending only on $d, \gamma$ such that

$$
\left|G_{\mathbf{a}}\left(x, t^{\prime}\right)-G_{\mathbf{a}}(x, t)\right| \leq \frac{\left[\Lambda\left|t-t^{\prime}\right|\right]^{\gamma} C_{d, \gamma}(\Lambda / \lambda)^{3 d+6}}{(\Lambda t)^{d / 2+\gamma}},
$$

provided $\left|t-t^{\prime}\right| \leq t / 2$.

Proof. This is the same as the proof of Lemma 5.8.

Next we show that one can analytically continue the function $q(\xi, \eta), \quad \xi \in$ $\mathbf{R}^{d}, \operatorname{Re}(\eta)>0$, in $\xi$. The analogue of Lemma 5.9 is the following:

Lemma 6.4. The function $q(\xi, \eta), \xi \in \mathbf{R}^{d}, \operatorname{Re}(\eta)>0$, extends analytically to the region $\mathbf{e}_{1} \cdot \xi \in \mathbf{C}$ satisfying

$$
\operatorname{Re}(\eta)>2 \Lambda \theta^{2}, \quad \theta=\operatorname{Im}\left(\mathbf{e}_{1} \cdot \xi\right) .
$$

Proof. We follow the argument of Lemma 5.9. If (6.6) holds, then there is the inequality

$$
\left|\eta+\Lambda \xi^{T} \xi\right| \geq \Lambda|\xi|^{2} .
$$

Thus $\left\|T_{\Lambda, \eta, \xi}\right\| \leq 1$ in the region (6.6), whence $q(\xi, \eta)$ is also analytic in this region. We also have the inequality

$$
\left|\eta+\Lambda \xi^{T} \xi\right| \geq|\operatorname{Im}(\eta)|-2 \Lambda|\beta||\theta|
$$

where $\mathbf{e}_{1} \cdot \xi=\beta+i \theta$. Adding (6.7), (6.8) we conclude that

$$
2\left|\eta+\Lambda \xi^{T} \xi\right| \geq|\operatorname{Im}(\eta)| .
$$

Combining this last inequality with (6.7) we obtain the analogue of (5.18), namely

$$
\left|\eta+\Lambda \xi^{T} \xi\right| \geq \frac{1}{3}\left[|\operatorname{Im}(\eta)|+\Lambda|\xi|^{2}\right]
$$

provided (6.6) holds.

Proof of Theorem 1.3. Note that Lemma 5.10 holds in the region (6.6). Hence one also has

$$
\operatorname{Re}[\eta+\xi q(\xi, \eta) \xi] \geq \lambda|\xi|^{2},
$$

in the region (6.6). The factor $1 / 8$ in the exponential bound in Theorem 1.3 comes from the fact that

$$
\inf _{\theta}\left[2 \Lambda \theta^{2} t+\theta\left(x \cdot \mathbf{e}_{1}\right)\right]=-\frac{\left(x \cdot \mathbf{e}_{1}\right)^{2}}{8 \Lambda t} .
$$

We are left then to establish (6.1). To do this we proceed as in Lemma 5.11, replacing summation over $x \in \mathbf{Z}^{d}$ by integration over $\mathbf{R}^{d}$. Note that one needs to establish that the distributional gradient $\nabla u(x, t, \omega)$ of $u(x, t, \omega)$ is indeed given by the formula $\partial v\left(x, t, \tau_{x, t} \omega\right)$, where $\partial v(x, t, \omega)$ is defined by the analogue of (5.26).

\section{ACKNowledgement}

This research was partially supported by NSF under grant DMS-0138519. 


\section{REFERENCES}

[1] D. G. Aronson, Non-negative solutions of linear parabolic equations, Ann. Sci. Norm. Sup. Pisa (3) 22 (1968) , 607-94. MR 55:8553

[2] J. Conlon, Homogenization of random walk in asymmetric random environment, New York J. Math. 8 (2002), 31-61. MR 1887697

[3] E. A. Carlen, S. Kusuoka and D. Stroock, Upper bounds for symmetric Markov transition functions, Ann. Inst. H. Poincaré 23 (1987), 245-87. MR 88i:35066

[4] J. Conlon and A. Naddaf, Green's functions for elliptic and parabolic equations with random coefficients, New York J. Math. 6 (2000), 153-225. MR 2001j:35282

[5] E. B. Davies, Heat Kernels and Spectral Theory, Cambridge Tracts in Mathematics 92, Cambridge University Press, Cambridge, New York, 1989. MR 90e:35123

[6] T. Delmotte and J. Deuschel, On estimating the derivatives of symmetric diffusions in stationary random environment, preprint 2003.

[7] G. Giacomin, S. Olla and H. Spohn, Equilibrium fluctuations for $\nabla \phi$ interface model, Ann. Probab. 29 (2001), 1138-1172. MR 2003c:60161

[8] C. Landim, S. Olla and H. T. Yau, Convection-diffusion equation with space-time ergodic random flow, Probab. Theory Relat. Fields 112 (1998), 203-220. MR 99j:35084

[9] M. Reed and B. Simon, Methods of Modern Mathematical Physics II: Fourier Analysis, SelfAdjointness, Academic Press, New York, London, 1975. MR 58:12429b

[10] E. Stein and G. Weiss, Introduction to Fourier Analysis on Euclidean Spaces, Princeton Mathematical Series, No. 32, Princeton University Press, Princeton, N.J., 1971. MR 46:4102

[11] V. Zhikov, S. Kozlov and O. Oleinik, Homogenization of Differential Operators and Integral Functionals, Springer-Verlag, Berlin, 1994. MR 96h:35003b

Department of Mathematics, University of Michigan, Ann Arbor, Michigan 481091109

E-mail address: conlon@umich.edu 